\title{
Combining structural rugosity and crystal packing comparison: A route to more polymorphs?
}

\author{
Riccardo Montis ${ }^{\mathrm{a}, \mathrm{b} *}$, Michael B. Hursthouse ${ }^{\mathrm{bc}}$, John Kendrick ${ }^{\mathrm{d}}$, Jason Howe ${ }^{\mathrm{b}}$ and Richard J. Whitby ${ }^{\mathrm{b}}$. \\ a Università degli Studi di Urbino "Carlo Bo", Via Aurelio Saffi, 2 - 61029 Urbino PU - Italy \\ ${ }^{b}$ University of Southampton, University Road, Southampton, SO17 1BJ, UK \\ 'University of Leeds, Leeds, LS2 9JT, UK
}

\section{Electronic Supplementary Information}

\section{S1. Synthetic Procedures}

\section{2-Methylbenzo[b]thiophene}

Benzothiophene ( $1 \mathrm{~g}, 7.45 \mathrm{mmol})$ was dissolved in THF $(50 \mathrm{~mL})$ under an atmosphere of argon and cooled to $-78^{\circ} \mathrm{C}$. $n$-butyllithium $\left(2.5 \mathrm{M} \mathrm{sol}^{\mathrm{n}}\right.$ in hexanes, $\left.3.88 \mathrm{~mL}, 9.69 \mathrm{mmol}\right)$ was slowly added and the reaction mixture was left stirring at $-78^{\circ} \mathrm{C}$ for 20 minutes. Iodomethane $(0.69 \mathrm{~mL}, 11.18 \mathrm{mmol})$ was added slowly keeping the temperature below $-70^{\circ} \mathrm{C}$. After 20 minutes the reaction mixture was allowed to warm to room temperature then sodium bicarbonate solution $(\sim 50 \mathrm{~mL})$ added and the product extracted into ethyl acetate. After drying $\left(\mathrm{MgSO}_{4}\right)$ and removal of solvent recrystallisation from hexane and small amount of ethyl acetate gave 2-methylbenzo[b]thiophene as light-yellow crystals $(0.53 \mathrm{~g}$, $3.55 \mathrm{mmol}, 48 \%)$. ${ }^{1} \mathrm{H}$ data consistent with the literature. ${ }^{1}$

${ }^{1} \mathrm{H}$ NMR $\left(300 \mathrm{MHz}, \mathrm{CDCl}_{3}\right) \delta 7.76(1 \mathrm{H}, \mathrm{d}, J=7.5 \mathrm{~Hz}), 7.66(1 \mathrm{H}, \mathrm{d}, J=7.2 \mathrm{~Hz}), 7.34-7.23(2 \mathrm{H}, \mathrm{m})$, $6.99(1 \mathrm{H}, \mathrm{brs}), 2.60(3 \mathrm{H}, \mathrm{d}, J=1.1 \mathrm{~Hz}) \mathrm{ppm}$.

\section{2-Methylbenzo[b]thiophene 1,1-dioxide}

2-Methylbenzo[b]thiophene (10) (0.52 g, $3.55 \mathrm{mmol})$ was dissolved in a solution of glacial acetic acid $(5 \mathrm{~mL})$ and hydrogen peroxide $(30 \%, 2.25 \mathrm{~mL}, 22.40 \mathrm{mmol})$ and heated to reflux. After 3 hours water $(10 \mathrm{~mL})$ was added and a solid formed which was filtered off. Purification by column chromatography (silica gel, 50\% ethyl acetate/hexane) followed by recrystallisation from hexane with a small amount of ethyl acetate gave 2-methylbenzo[b]thiophene 1,1-dioxide as light green needles (0.306 g, $1.698 \mathrm{mmol}$, $48 \%)$. Data consistent with the literature. ${ }^{2,3}$

${ }^{1} \mathrm{H}$ NMR $\left(300 \mathrm{MHz}, \mathrm{CDCl}_{3}\right) \delta 7.72(1 \mathrm{H}, \mathrm{d}, J=7.3 \mathrm{~Hz}), 7.55-7.42(2 \mathrm{H}, \mathrm{m}), 7.28(1 \mathrm{H}, \mathrm{d}, J=7.7 \mathrm{~Hz})$, $6.78(1 \mathrm{H}$, brs $), 2.23(3 \mathrm{H}, \mathrm{d}, J=1.8 \mathrm{~Hz}) \mathrm{ppm} .{ }^{13} \mathrm{C} \mathrm{NMR}\left(75 \mathrm{MHz}, \mathrm{CDCl}_{3}\right) \delta 140.98(\mathrm{C}), 136.51(\mathrm{C})$, $133.57(\mathrm{CH}), 131.65(\mathrm{C}), 129.31(\mathrm{CH}), 125.86(\mathrm{CH}), 124.25(\mathrm{CH}), 121.55(\mathrm{CH}), 9.08\left(\mathrm{CH}_{3}\right) \mathrm{ppm}$. LRMS (GC/EI) m/z $180(80 \%)(\mathrm{M})^{+}, 137$ (100\%), 131 (66\%), 115 (75\%), 109 (75\%). IR (neat) $\square \max$ $2359(\mathrm{w}), 2158(\mathrm{w}), 1448(\mathrm{w}), 1282(\mathrm{~m}), 1141(\mathrm{~m}) \mathrm{cm}^{-1}$. 


\section{3-Bromobenzo[b]thiophene}

Benzothiophene $(1 \mathrm{~g}, 7.45 \mathrm{mmol})$ was dissolved in a solution of glacial acetic acid $(7.5 \mathrm{~mL})$ and chloroform $(7.5 \mathrm{~mL})$ at $0^{\circ} \mathrm{C}$. N-bromosuccinimide $(1.66 \mathrm{~g}, 9.31 \mathrm{mmol})$ was added over a period of 90 minutes. Reaction mixture allowed to warm to room temperature and stirred for 20 hours. Upon completion reaction mixture was washed with saturated sodium thiosulphate solution followed by saturated sodium carbonate solution. Product was then filtered through a short pad of silica to give 3bromobenzo[b]thiophene as a brown oil $(1.44 \mathrm{~g}, 6.76 \mathrm{mmol}, 91 \%) .{ }^{1} \mathrm{H}$ data consistent with the literature. ${ }^{4}$ Crude material was used in the next step.

${ }^{1} \mathrm{H}$ NMR $\left(300 \mathrm{MHz}, \mathrm{CDCl}_{3}\right) \delta$ 7.88-7.84 (2H, m), 7.51-7.40 (3H, m) ppm.

\section{3-Methylbenzo[b]thiophene and 3-bromo-2-methylbenzo[b]thiophene}

Crude 3-bromobenzo[b]thiophene $(1.44 \mathrm{~g}, 6.76 \mathrm{mmol})$ was dissolved in THF $(50 \mathrm{~mL})$ under an atmosphere of argon and cooled to $-78^{\circ} \mathrm{C}$. $n$-butyllithium $\left(2.5 \mathrm{M} \mathrm{sol}^{\mathrm{n}}\right.$ in hexanes, $\left.2.70 \mathrm{~mL}, 6.76 \mathrm{mmol}\right)$ was slowly added and the reaction mixture was left stirring at $-78^{\circ} \mathrm{C}$ for 20 minutes. Iodomethane $(0.51$ $\mathrm{mL}, 8.11 \mathrm{mmol}$ ) was added slowly keeping the temperature below $-70^{\circ} \mathrm{C}$. After 20 minutes the reaction mixture was allowed to warm to room temperature, sodium bicarbonate solution ( $\sim 50 \mathrm{~mL})$ added, and the product extracted with ethyl acetate. Purification by column chromatography (silica gel, $100 \%$ hexane) gave 3-methylbenzo[b]thiophene as pale-yellow oil (0.66 g, $4.44 \mathrm{mmol}, 66 \%)$ (analysis showed $\sim 80 \%$ purity) and 3-bromo-2-methylbenzo[b]thiophene $(0.18 \mathrm{~g}, 0.79 \mathrm{mmol}, 12 \%) .{ }^{1} \mathrm{H}$ data consistent with the literature. ${ }^{1,5}$

\section{3-Bromo-2-methylbenzo[b]thiophene}

${ }^{1} \mathrm{H}$ NMR $\left(300 \mathrm{MHz}, \mathrm{CDCl}_{3}\right) \delta 7.74(1 \mathrm{H}, \mathrm{d}, J=5.5 \mathrm{~Hz}), 7.72(1 \mathrm{H}, \mathrm{d}, J=4.8 \mathrm{~Hz}), 7.42(1 \mathrm{H}, \mathrm{t}, J=7.0$ $\mathrm{Hz}), 7.34(1 \mathrm{H}, \mathrm{t}, J=7.3 \mathrm{~Hz}), 2.57(3 \mathrm{H}, \mathrm{s}) \mathrm{ppm}$.

\section{3-Methylbenzo[b]thiophene}

${ }^{1} \mathrm{H}$ NMR (300 MHz, $\left.\mathrm{CDCl}_{3}\right) \delta 7.87(1 \mathrm{H}, \mathrm{dd}, J=7.3,1.8 \mathrm{~Hz}), 7.74(1 \mathrm{H}, \mathrm{dd}, J=7.1,1.6 \mathrm{~Hz}), 7.44-7.33$ $(2 \mathrm{H}, \mathrm{m}), 7.09(1 \mathrm{H}, \mathrm{d}, J=0.7 \mathrm{~Hz}), 2.47(3 \mathrm{H}, \mathrm{d}, J=1.5 \mathrm{~Hz}) \mathrm{ppm}$.

\section{3-Methylbenzo[b]thiophene 1,1-dioxide}

3-Methylbenzo[b]thiophene $(0.30 \mathrm{~g}, 2.00 \mathrm{mmol})$ was dissolved in a solution of glacial acetic acid (5 $\mathrm{mL})$ and hydrogen peroxide $(30 \%, 1.26 \mathrm{~mL}, 12.60 \mathrm{mmol})$ and heated to reflux. After 30 minutes water $(10 \mathrm{~mL})$ was added and a solid formed which was filtered off. Purification by column chromatography (silica gel, 0-30\% ethyl acetate/hexane) gave title thiophene dioxide as a white solid (0.145 g, 0.80 mmol, 40\%). Data is consistent with the literature. ${ }^{2,3}$

${ }^{1} \mathrm{H}$ NMR $\left(300 \mathrm{MHz}, \mathrm{CDCl}_{3}\right) \delta 7.72(1 \mathrm{H}, \mathrm{d}, J=7.3 \mathrm{~Hz}), 7.63-7.52(2 \mathrm{H}, \mathrm{m}), 7.42(1 \mathrm{H}, \mathrm{d}, J=7.3 \mathrm{~Hz})$, $6.48(1 \mathrm{H}, \mathrm{brs}), 2.28(3 \mathrm{H}, \mathrm{d}, J=0.7 \mathrm{~Hz}) \mathrm{ppm} .{ }^{13} \mathrm{C} \mathrm{NMR}\left(75 \mathrm{MHz}, \mathrm{CDCl}_{3}\right) \delta 142.78(\mathrm{C}), 137.54(\mathrm{C})$, 
$133.44(\mathrm{CH}), 133.09(\mathrm{C}), 130.39(\mathrm{CH}), 125.76(\mathrm{CH}), 122.23(\mathrm{CH}), 120.90(\mathrm{CH}), 13.81\left(\mathrm{CH}_{3}\right) \mathrm{ppm}$. LRMS (GC/EI) m/z $180(79 \%)(\mathrm{M})^{+}, 151$ (100\%), 131 (62\%), 115 (53\%). IR (neat) $\mathrm{n}_{\max } 3091$ (w), 2360 (w), $1377(\mathrm{w}), 1286(\mathrm{~m}), 1181(\mathrm{~s}), 1104(\mathrm{~m}) \mathrm{cm}^{-1}$.

\section{2,3-Dimethylbenzo[b]thiophene ${ }^{6}$}

$\mathrm{Cp}_{2} \mathrm{ZrCl}_{2}(0.5 \mathrm{~g}, 1.7 \mathrm{mmol})$ was dissolved in THF $(8 \mathrm{~mL})$ and cooled to $-40{ }^{\circ} \mathrm{C}$. $\mathrm{PhLi}(1.8 \mathrm{M}, 1.92 \mathrm{~mL}$, $3.45 \mathrm{mmol}$ ) was added slowly then stirred for 1 hour at $-40{ }^{\circ} \mathrm{C}$. The reaction mixture was allowed to warm to room temperature then transferred to a Schlenk tube sealed with a J. Young tap. 2-Butyne (0.26 $\mathrm{mL}, 3.4 \mathrm{mmol}$ ) was added and the solution degassed with 2 freeze-evacuate-thaw cycles, then heated to $80{ }^{\circ} \mathrm{C}$ for 24 hours. After cooling to $-78{ }^{\circ} \mathrm{C} \mathrm{S}_{2} \mathrm{Cl}_{2}(0.2 \mathrm{~mL}, 2.5 \mathrm{mmol})$ was added then stirred for 10 minutes before quenching with $\mathrm{HCl}_{(\mathrm{aq})}(1 \mathrm{M}, 0.5 \mathrm{~mL})$. After warming to room temperature $\mathrm{THF}$ was removed under vacuum and the solid residue extracted with hexane. Removal of solvent gave 2,3dimethylbenzo[b]thiophene (0.294 g, quant.) as a yellow oil. The crude product was used directly in next reaction. ${ }^{1} \mathrm{H}$ NMR data consistent with the literature. ${ }^{6}$

${ }^{1} \mathrm{H}$ NMR $\left(300 \mathrm{MHz}, \mathrm{CDCl}_{3}\right) \delta 7.75(1 \mathrm{H}, \mathrm{d}, J=7.7 \mathrm{~Hz}), 7.60(1 \mathrm{H}, \mathrm{d}, J=7.7 \mathrm{~Hz}), 7.38-7.25(2 \mathrm{H}, \mathrm{m})$, $2.50(3 \mathrm{H}, \mathrm{s}), 2.31(3 \mathrm{H}, \mathrm{s}) \mathrm{ppm}$.

\section{2,3-Dimethylbenzo[b]thiophene 1,1-dioxide}

Crude 2,3-dimethylbenzo[b]thiophene $(0.29 \mathrm{~g}, 1.81 \mathrm{mmol})$ was dissolved in a solution of glacial acetic acid $(10 \mathrm{~mL})$ and hydrogen peroxide $(30 \%, 0.98 \mathrm{~mL}, 10.9 \mathrm{mmol})$ and heated to reflux. After 1.5 hours, water $(10 \mathrm{~mL})$ was added and a solid formed which was filtered off. Purification by column chromatography (silica gel, 0-30\% ethyl acetate/hexane) gave title thiophene dioxide as a white solid (89 mg, $0.46 \mathrm{mmol}, 25 \%)$. Data is consistent with the literature..$^{2,3}$

${ }^{1} \mathrm{H}$ NMR $\left(300 \mathrm{MHz}, \mathrm{CDCl}_{3}\right) \delta 7.73(1 \mathrm{H}, \mathrm{d}, J=7.3 \mathrm{~Hz}), 7.60-7.55(1 \mathrm{H}, \mathrm{m}), 7.49-7.44(1 \mathrm{H}, \mathrm{m}), 7.38$ $(1 \mathrm{H}, \mathrm{d}, J=7.7 \mathrm{~Hz}), 2.16(6 \mathrm{H}, \mathrm{s}) \mathrm{ppm} .{ }^{13} \mathrm{C} \mathrm{NMR}\left(75 \mathrm{MHz}, \mathrm{CDCl}_{3}\right) \delta 133.47(\mathrm{CH}), 129.14(\mathrm{CH}), 121.50$ $(\mathrm{CH}), 120.95(\mathrm{CH}), 10.92\left(\mathrm{CH}_{3}\right), 6.85\left(\mathrm{CH}_{3}\right)$ ppm. LRMS (GC/EI) m/z $194(26 \%)(\mathrm{M})^{+}, 162(28 \%)$, 151 (100\%), 147 (37\%), 115 (29\%). IR (neat) $\square$ max 2978 (w), 2360 (w), 1442 (w), 1280 (s), 1170 (s), $1150(\mathrm{~s}) \mathrm{cm}^{-1}$. 


\section{S2. Experimental Characterization}

Table S1 DSC data for the three methyl derivatives of benzothiophene-1,1-dioxide (0-200@10 $\left.{ }^{\circ} \mathrm{C} . \mathrm{min}^{-1}\right)$. The values are reported as averaged of three individual measurements.

\begin{tabular}{lccc}
\hline & & DSC $_{\text {Onset }}\left({ }^{\circ} \mathbf{C}\right)$ & DSC $_{\text {Peak }}\left({ }^{\circ} \mathbf{C}\right)$ \\
\hline $2-\mathrm{Me}$ & & $109.4 \pm 0.5$ & $111.2 \pm 0.6$ \\
& & & \\
$3-\mathrm{Me}$ & I & $142.1 \pm 0.1$ & $143.7 \pm 0.3$ \\
& II & $145.7 \pm 0.5$ & $146.3 \pm 0.4$ \\
& & & \\
$23-\mathrm{Me}$ & I & $147.2 \pm 0.3$ & $149.4 \pm 0.3$ \\
& II & $151.2 \pm 0.4$ & $152.7 \pm 0.2$ \\
\hline
\end{tabular}

To investigate in more detail the two melting events observed for 3-Me and 23-Me, a hot-stage microscopy (HSM) analysis was carried out reproducing the experimental conditions used for the DSC (Figure S2 a-f and Figure S3 a-f).

For 3-Me we observed a thermal event in the range 139-140 (red circle in Figure S2 c) followed by a melting process $144-147^{\circ} \mathrm{C}$ (Figure S2 e and f).

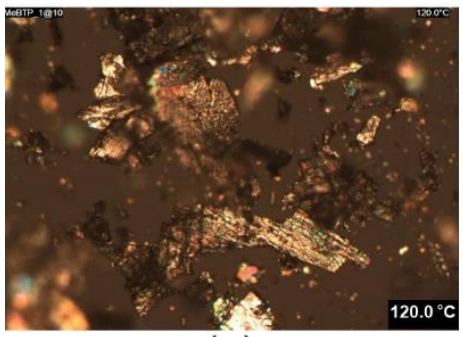

(a)

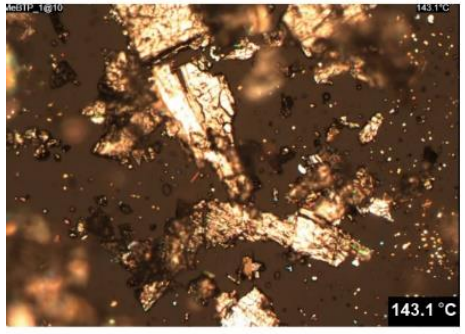

(d)

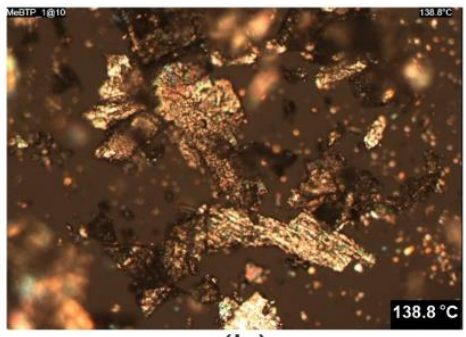

(b)

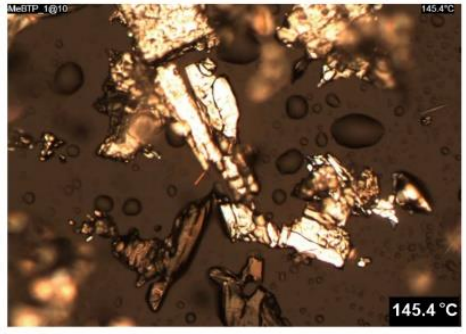

(e)

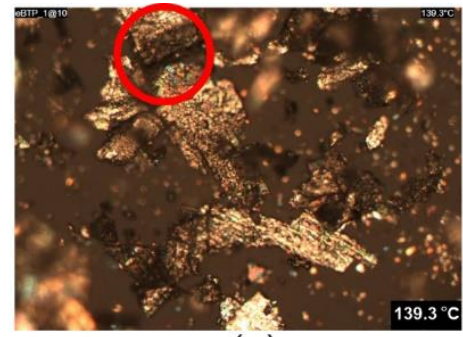

(c)

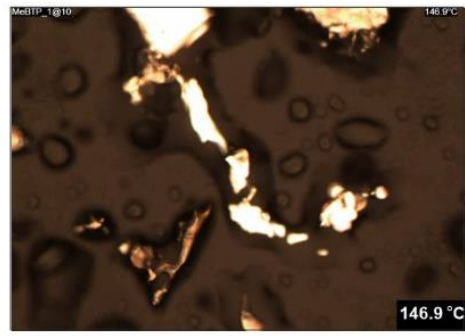

(f)

Figure S2. HSM (heating rate: $10^{\circ} \mathrm{C} / \mathrm{min}$ ) for 3-Me.

A similar behaviour was observed for 23-Me, which showed some changes in the range 146-148 (compare Figure S3 c and d, red circles) melting process in the range $150-152{ }^{\circ} \mathrm{C}$ (Figure S3 f). 


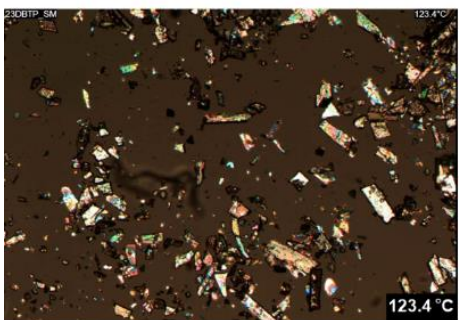

(a)

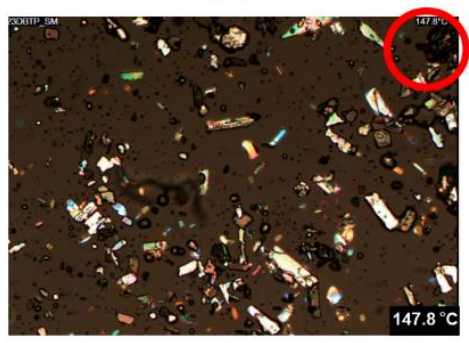

(d)

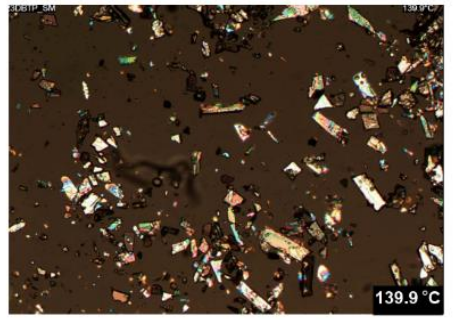

(b)

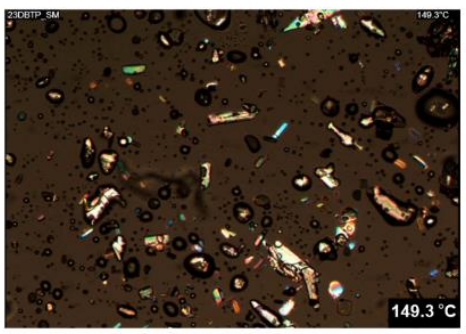

(e)

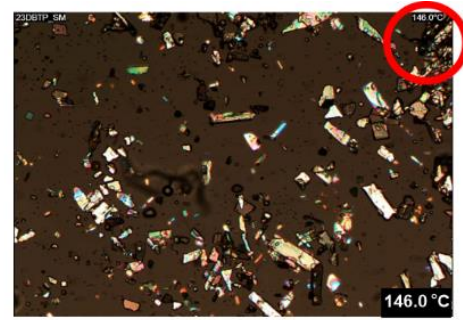

(c)

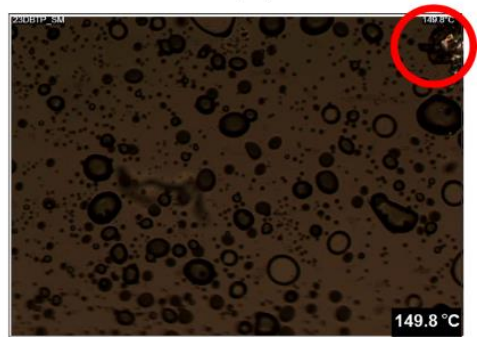

(f)

Figure S3. HSM (heating rate: $10{ }^{\circ} \mathrm{C} / \mathrm{min}$ ) for $23-\mathrm{Me}$.

Interestingly, during this analysis both the samples showed the tendency to nicely crystallise as single crystals, simply by slowly cooling the melt (see Figure S4 c and d).

Table S2. Crystallization from solvents and from melt

\begin{tabular}{|c|c|c|c|c|}
\hline crystallization method & solvent & $2-\mathrm{Me}^{\mathrm{a}, \mathrm{b}}$ & $3-\mathrm{Me}^{\mathrm{a}}$ & 23-Me ${ }^{\mathrm{a}}$ \\
\hline \multirow{9}{*}{ slow evaporation } & hexane/ AcOOEt & 2-Me & 3-Me $\alpha$ & 23-Me $\alpha$ \\
\hline & $\mathrm{MeOH}$ & 2-Me & 3 -Me $\alpha$ & 23-Me $\alpha$ \\
\hline & $\mathrm{EtOH}$ & 2-Me & 3-Me $\alpha$ & 23-Me $\alpha$ \\
\hline & PropOH & 2-Me & 3 -Me $\alpha$ & $23-\mathrm{Me} \alpha$ \\
\hline & AcOOEt & 2-Me & 3-Me $\alpha$ & 23-Me $\alpha$ \\
\hline & $\mathrm{MeCN}$ & 2-Me & $3-\operatorname{Me} \alpha$ & 23-Me $\alpha$ \\
\hline & $\mathrm{MeCN} / \mathrm{H}_{2} \mathrm{O} 1: 1$ & 2-Me & 3 -Me $\alpha$ & 23-Me $\alpha$ \\
\hline & $\mathrm{MeOH} / \mathrm{H}_{2} \mathrm{O} 1: 1$ & 2-Me & 3-Me $\alpha$ & 23-Me $\alpha$ \\
\hline & DCM & 2-Me & $3-\operatorname{Me} \alpha$ & 23-Me $\alpha$ \\
\hline from the melt & - & 2-Me & 3-Me $\beta$ & 3-Me $\beta$ \\
\hline
\end{tabular}

${ }^{a}$ Different phases were characterized by single-crystal X-ray diffraction (unit cell determination) and by DSC.

${ }^{\text {b } C r y s t a l l i z a t i o n s ~ a l w a y s ~ p r o d u c e d ~ t h e ~ k n o w n ~ c r y s t a l ~ s t r u c t u r e ~(C S D ~ r e f ~ c o d e: ~ G A K P E N) ~}{ }^{7}$ 


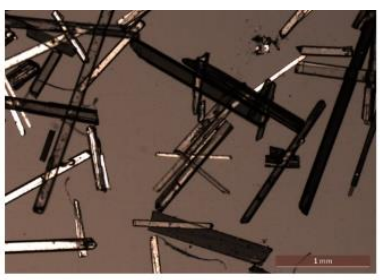

(a)

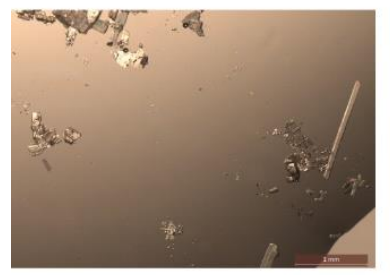

(b)

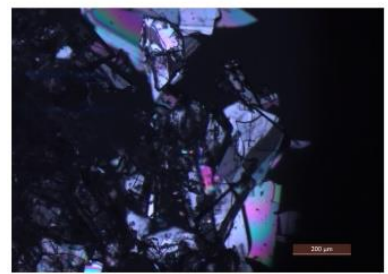

(d)

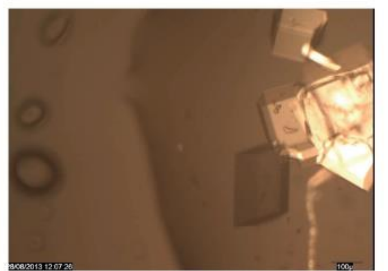

(c)

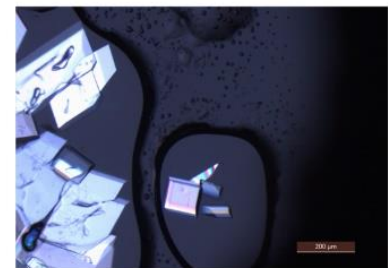

(e)

Figure S4. Crystal morphologies for (a) 2-Me; (b) 3-Me $\alpha$; (c) 3-Me $\beta$; (d) 23-Me $\alpha$; (e) 23-Me $\beta$

Table S3. Crystal data for phase 1-4

\begin{tabular}{|c|c|c|}
\hline & 3-Me $\beta$ & 23-Me $\beta$ \\
\hline CCDC deposition $\mathrm{N}$ & 2111348 & 2111347 \\
\hline Empirical formula & $\mathrm{C}_{9} \mathrm{H}_{8} \mathrm{O}_{2} \mathrm{~S}$ & $\mathrm{C}_{10} \mathrm{H}_{10} \mathrm{O}_{2} \mathrm{~S}$ \\
\hline Formula weight & 180.21 & 194.24 \\
\hline Crystal system & Monoclinic & Monoclinic \\
\hline Space group & $P 2_{1} / n$ & $C 2 / c$ \\
\hline$a(\AA)$ & $7.2483(3)$ & $11.3081(3)$ \\
\hline$b(\AA)$ & $8.0417(4)$ & $10.9999(2)$ \\
\hline$c(\AA)$ & $14.1246(7)$ & $16.1373(5)$ \\
\hline$\alpha\left(^{\circ}\right)$ & 90 & 90 \\
\hline$\beta\left(^{\circ}\right)$ & $92.028(3)$ & $106.099(2)$ \\
\hline$\gamma\left({ }^{\circ}\right)$ & 90 & 90 \\
\hline Volume $\left(\AA^{3}\right)$ & $822.79(7)$ & 1928.57(9) \\
\hline $\mathrm{T}(\mathrm{K})$ & $120(2)$ & $120(2)$ \\
\hline$Z / Z^{\prime}$ & $4 / 1$ & $8 / 1$ \\
\hline$D_{\text {calc }}\left(\mathrm{Mg} / \mathrm{m}^{3}\right)$ & 1.455 & 1.338 \\
\hline$\mu\left(\mathrm{mm}^{-1}\right)$ & 0.343 & 0.298 \\
\hline$\theta_{\min }-\theta_{\max }\left({ }^{\circ}\right)$ & $3.206-27.553$ & $2.91-27.48$ \\
\hline Refl.col/unique/ $R_{\text {int }}$ & $10286 / 1884$ / 0.0512 & $9517 / 2207 / 0.0678$ \\
\hline$R_{1} / w R_{2}(I>2 \sigma(I))$ & $0.0397 / 0.0866$ & $0.0519 / 0.1147$ \\
\hline$R_{1} / w R_{2}$ (all data) & $0.0541 / 0.0932$ & $0.0663 / 0.1254$ \\
\hline GoF & 1.029 & 1.067 \\
\hline
\end{tabular}




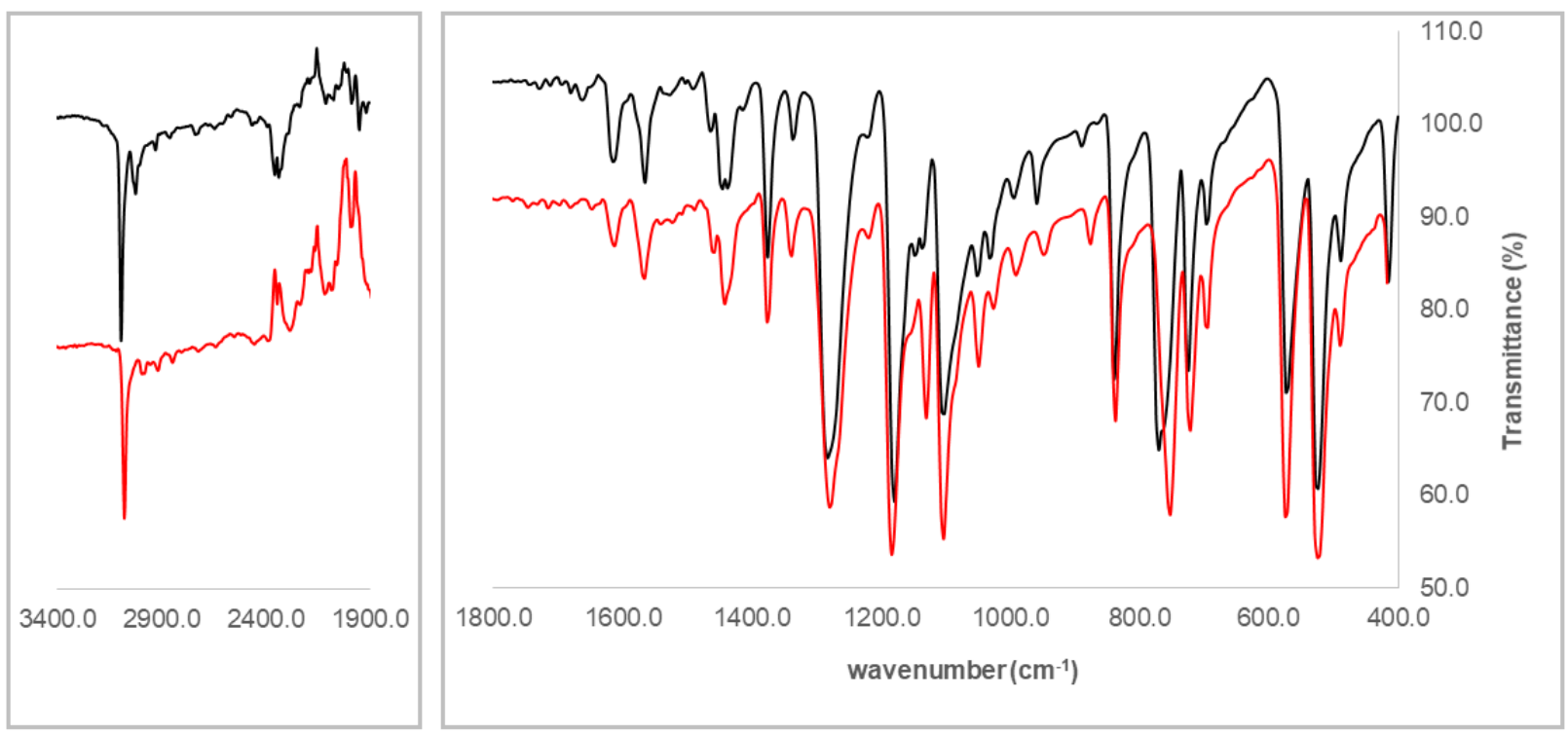

Figure S5. FT-IR spectra for 3-Me $\alpha$ (black) and 3-Me $\beta$ (red).

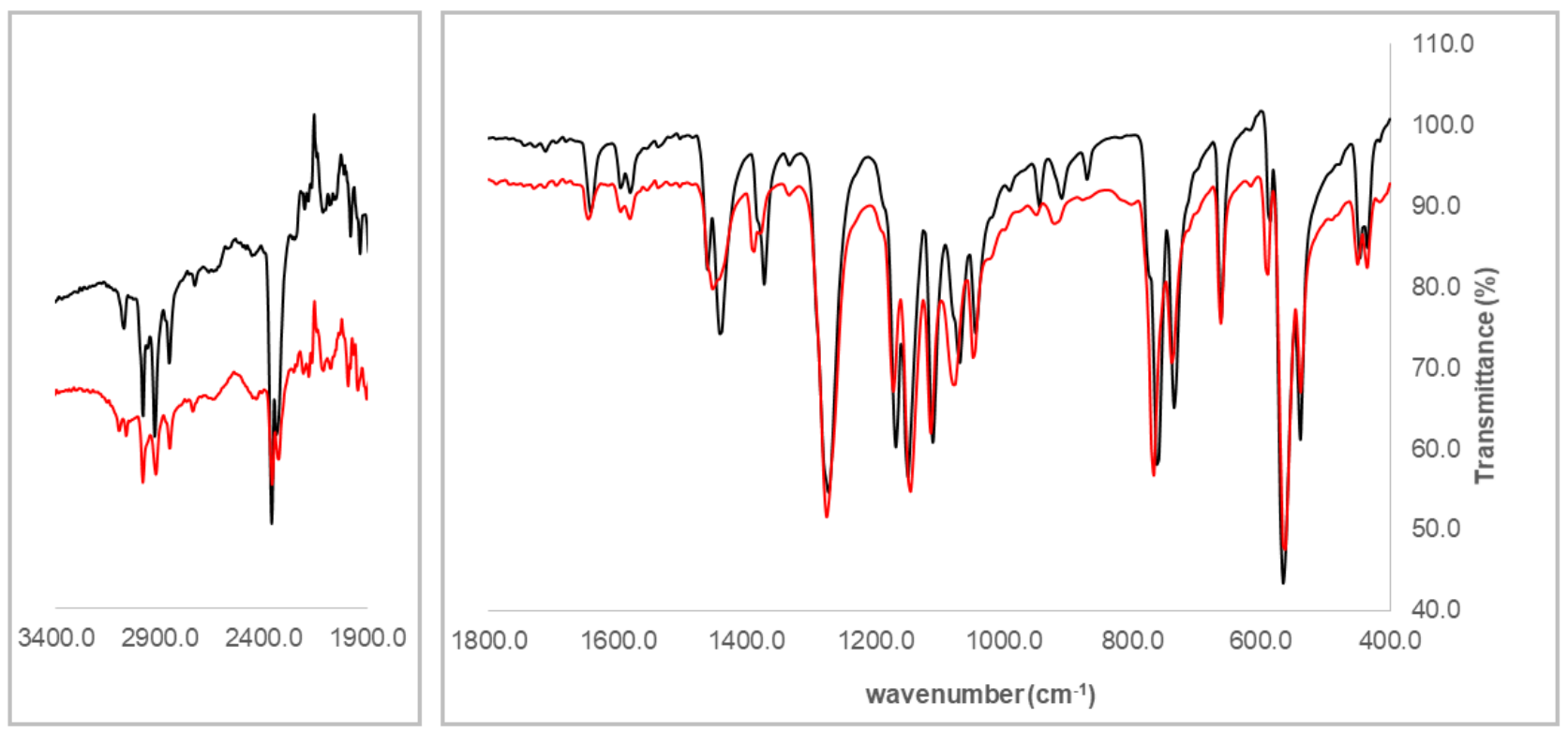

Figure S6. FT-IR spectra for 23-Me $\alpha$ (black) and 23-Me $\beta$ (red) 


\section{S3. XPac Analysis}

Table S4. Comparison 2-Me01-10 / 2-Me01-10

\begin{tabular}{|c|c|c|}
\hline \multicolumn{3}{|c|}{ COSP } \\
\hline Comparison & Results & Description \\
\hline 2-Me01 / 2-Me02 & 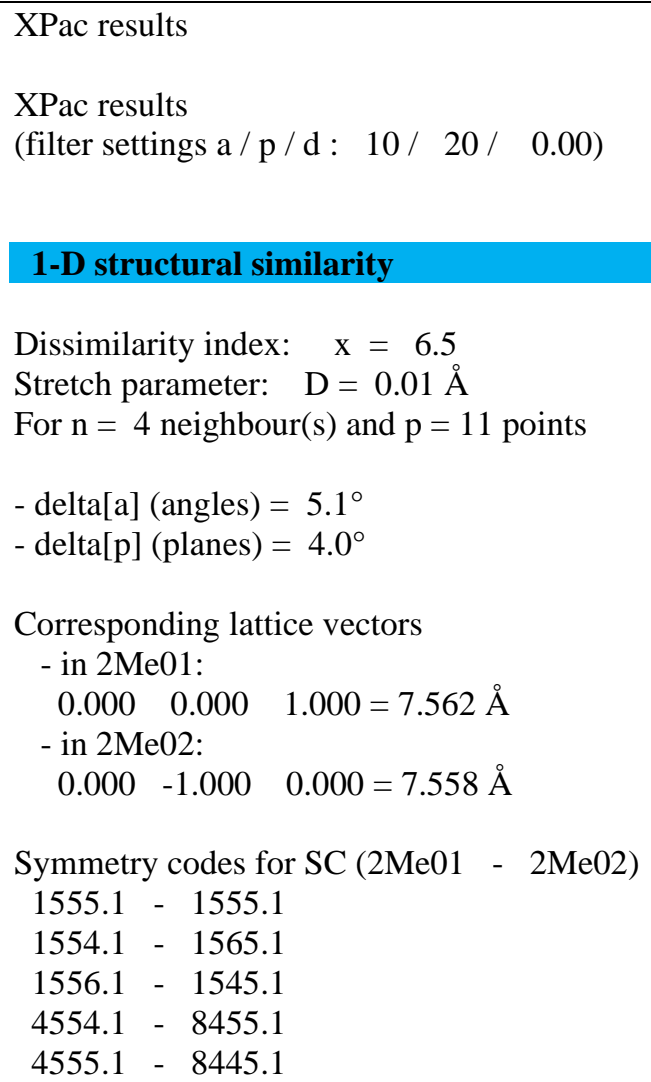 & Similarity S1 \\
\hline
\end{tabular}




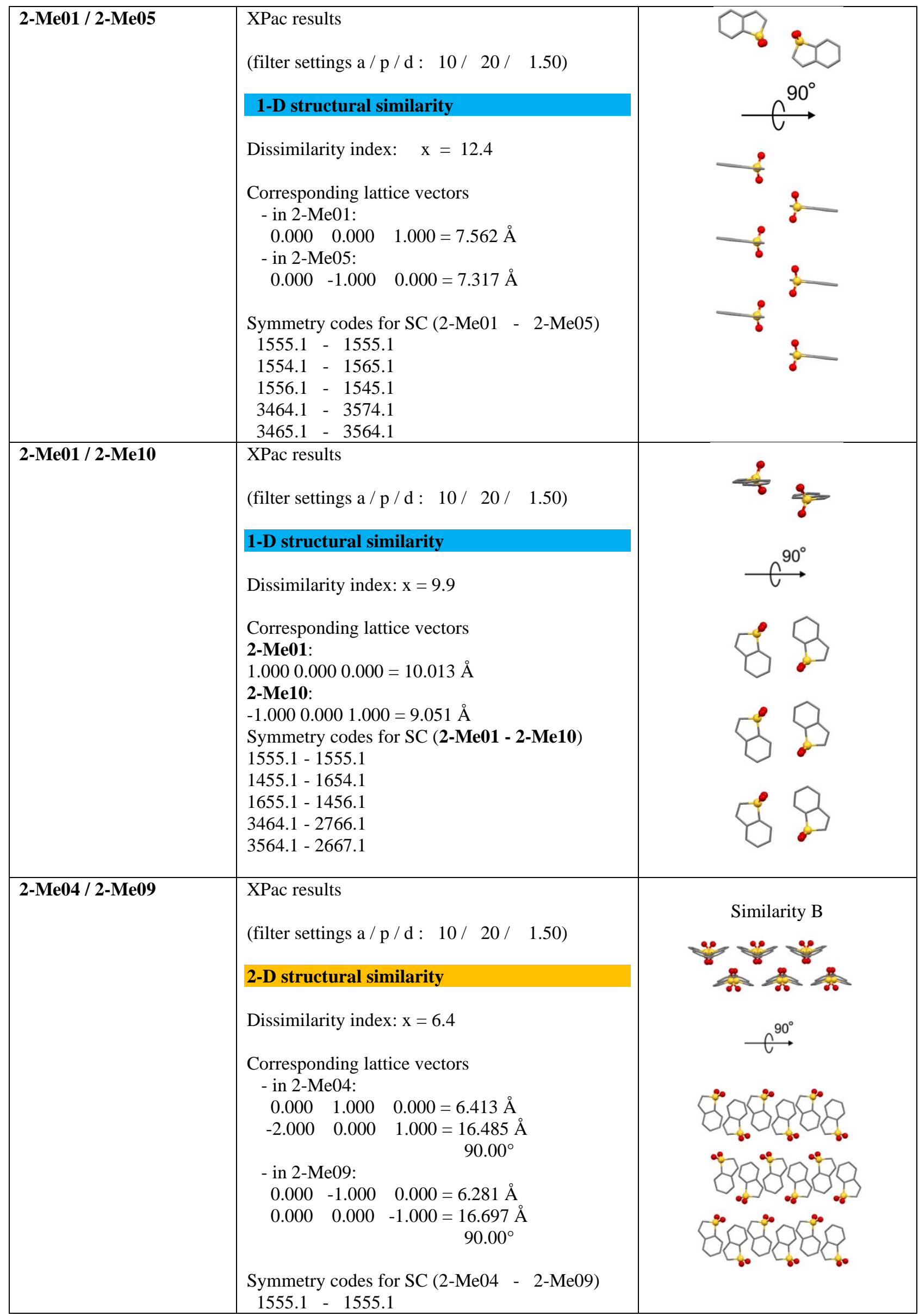




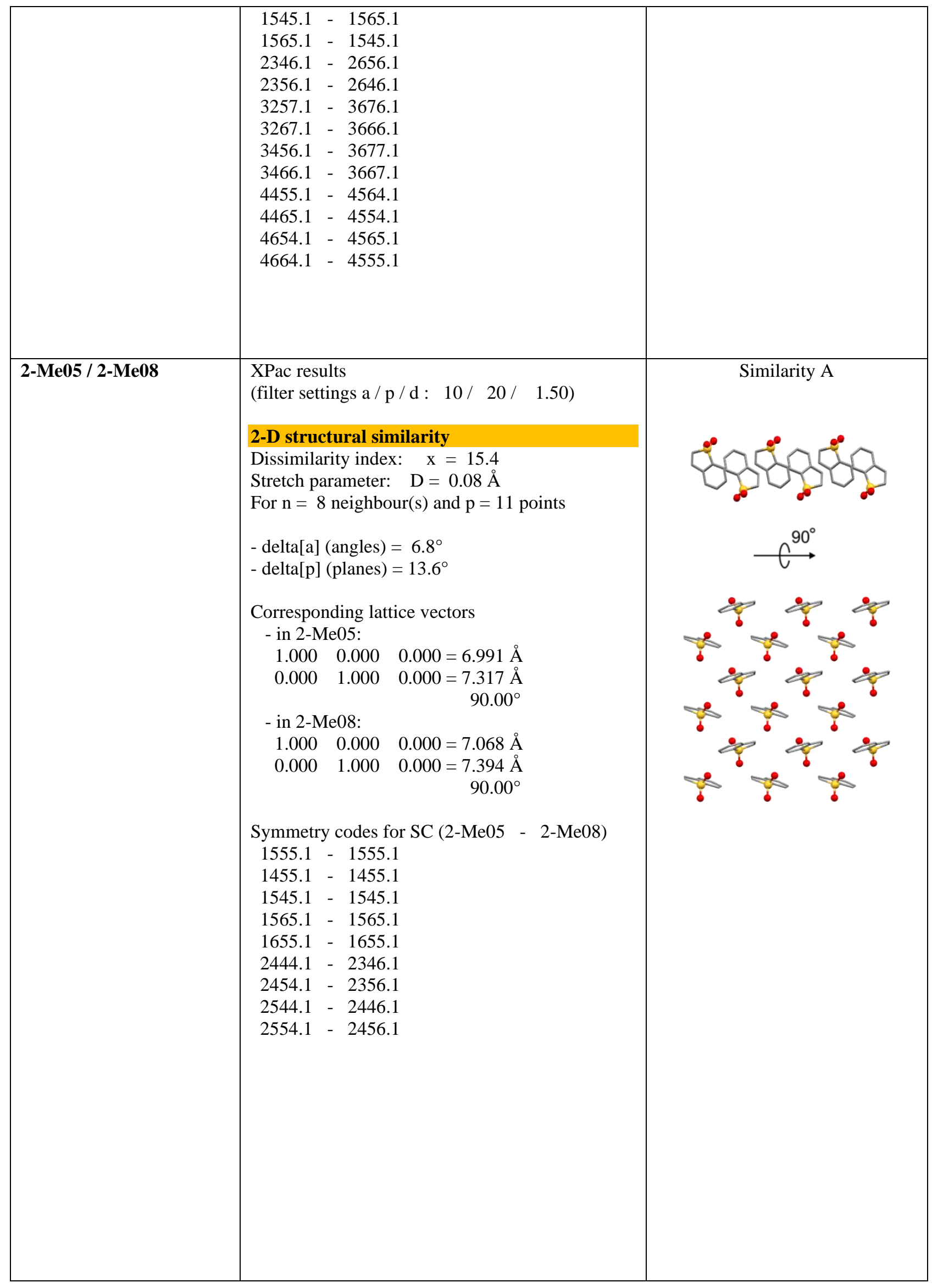




\begin{tabular}{|l|l|l|l|}
\hline 2-Me06 / 2-Me10 & 2-D structural similarity* \\
Corresponding lattice vectors \\
- - in 2-Me06: \\
$-1.0000 .0000 .000=7.800 \AA$ \\
$1.0000 .0001 .000=8.966 \AA$ \\
- in 2-Me10: \\
$0.000 \quad-1.000 \quad 0.000=7.621 \AA$ \\
$-1.000 \quad 0.000 \quad 1.000=9.051 \AA$
\end{tabular}

Table S5. Comparison 3-Me01-10 / 3-Me01-10.

\begin{tabular}{|c|c|c|}
\hline \multicolumn{3}{|c|}{ COSP } \\
\hline Comparison & Results & Description \\
\hline 3-Ме01 - 3-Ме03 & $\begin{array}{l}\text { XPac results } \\
\text { (filter settings a / p / }: 10 \text { / } 14 \text { / 1.50) } \\
\text { 1-D structural similarity } \\
\text { Dissimilarity index: } \mathrm{x}=2.6 \\
\text { Corresponding lattice vectors } \\
\text { 3-Me01: } \\
0.0000 .0001 .000=7.660 \AA \\
\text { 3-Me03: } \\
0.000 \text { - } 1.0000 .000=7.619 \AA \\
\text { Symmetry codes for SC (3-Me01 - 3-Me03) } \\
\text { 1555.1 - } 1555.1 \\
\text { 4554.1 - } 8355.1 \\
4555.1 \text { - } 8345.1\end{array}$ & Similarity S1 \\
\hline
\end{tabular}




\begin{tabular}{|c|c|c|}
\hline 3-Me02 - 3-Me04 & 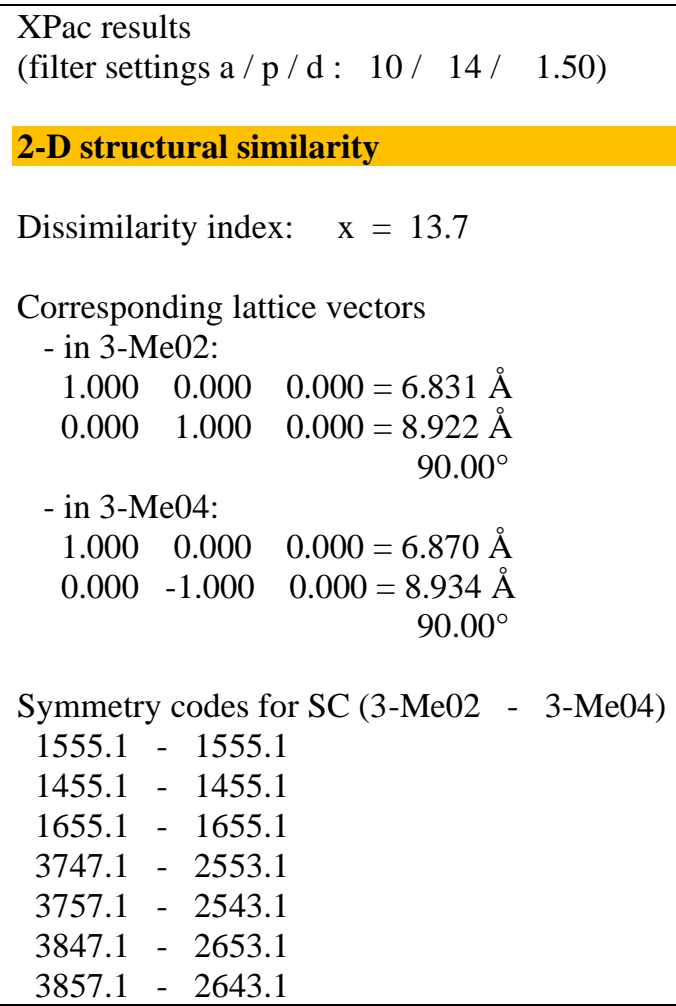 & Similarity F \\
\hline 3-Me03 - 3-Me05 & 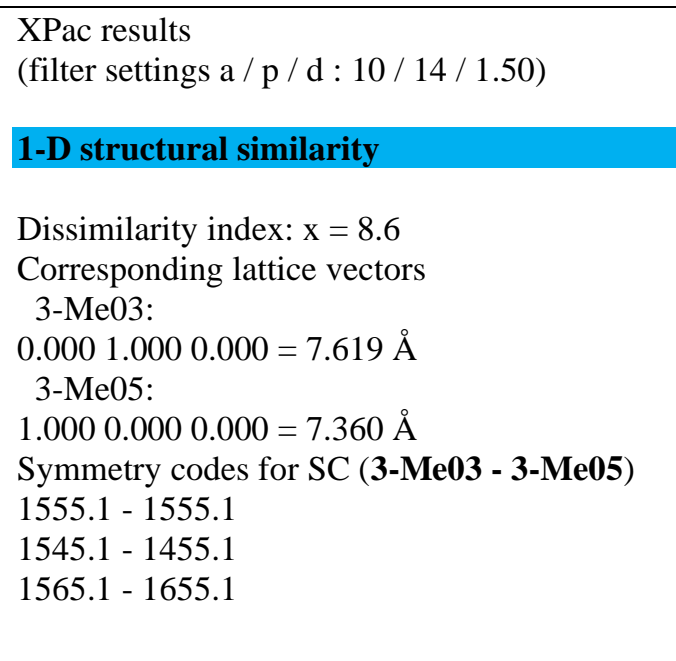 & 20 \\
\hline 3-Me03 - 3-Мe06 & 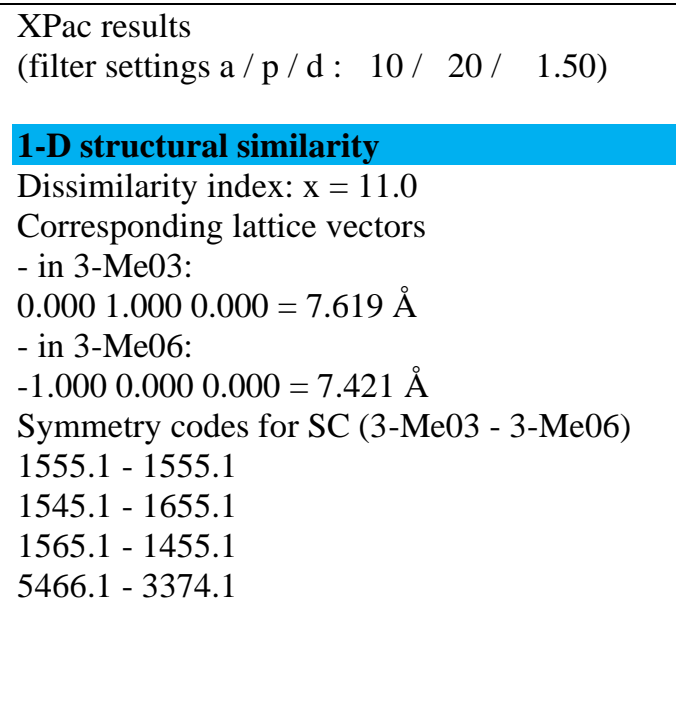 & b \\
\hline
\end{tabular}




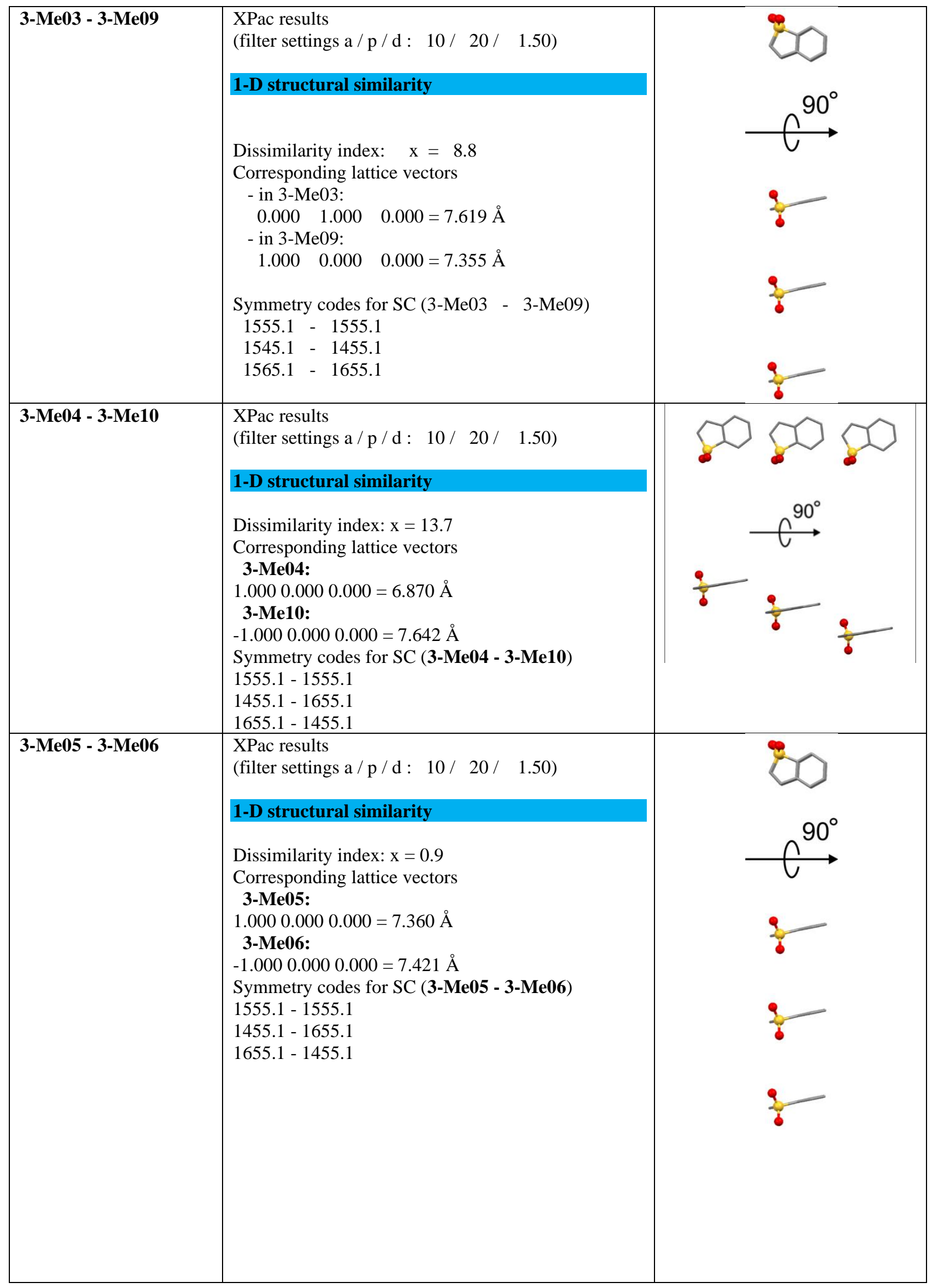




\begin{tabular}{|c|c|c|}
\hline 3-Me05 - 3-Me08 & 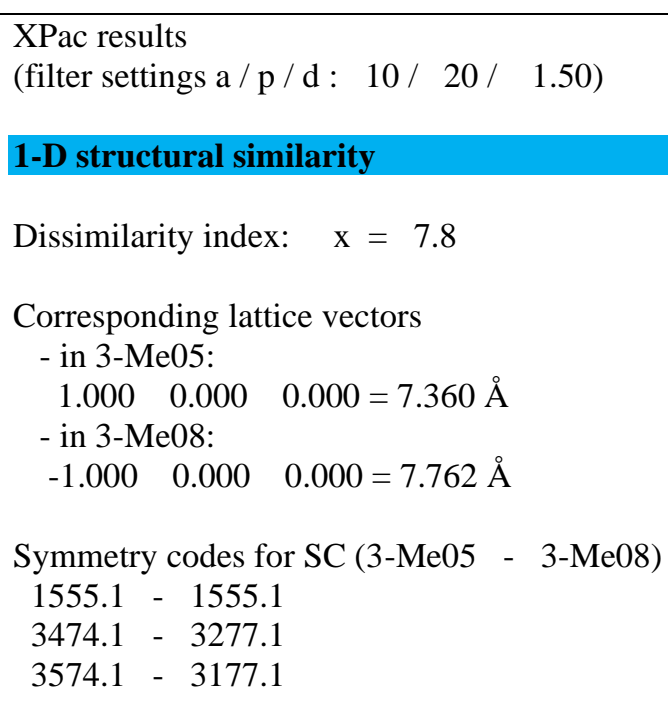 & Similarity S2 \\
\hline 3-Me05 - 3-Me09 & $\begin{array}{l}\text { XPac results } \\
\text { (filter settings a / p / : } 10 / 20 / 1.50) \\
\text { 1-D structural similarity } \\
\text { Dissimilarity index: } \mathrm{x}=1.9 \\
\text { Corresponding lattice vectors } \\
\text { 3-Me05: } \\
\text { 1.000 0.000 } 0.000=7.360 \AA \\
\text { 3-Me09: } \\
\text { 1.000 } 0.0000 .000=7.355 \AA \\
\text { Symmetry codes for SC (3-Me05 - 3-Me09) } \\
1555.1-1555.1 \\
1455.1-1455.1 \\
1655.1-1655.1\end{array}$ & \\
\hline 3-Me06 - 3-Me08 & $\begin{array}{l}\text { XPac results } \\
\text { (filter settings a / p / d : } 10 / 20 / 1.50) \\
\text { 1-D structural similarity } \\
\text { Dissimilarity index: } \mathrm{x}=8.0 \\
\text { Corresponding lattice vectors } \\
\text { 3-Me06: } \\
\text { 1.000 0.000 } 0.000=7.421 \AA \\
\text { 3-Me08: } \\
\text { 1.000 } 0.0000 .000=7.762 \AA \\
\text { Symmetry codes for SC (3-Me06 - 3-Me08) } \\
1555.1 \text { - } 1555.1 \\
3264.1 \text { - } 3177.1 \\
3364.1 \text { - } 3277.1\end{array}$ & Similarity S2 \\
\hline
\end{tabular}




\begin{tabular}{|c|c|c|}
\hline 3-Me06 - 3-Me09 & 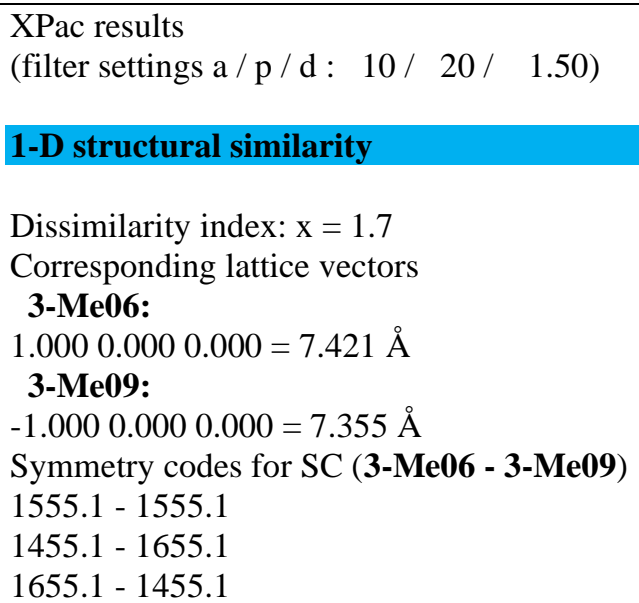 & \\
\hline 3-Me07 - 3-Me08 & $\begin{array}{l}\text { XPac results } \\
\text { (filter settings a / p / : } 10 / 20 / 1.50) \\
\text { 1-D structural similarity } \\
\text { Dissimilarity index: } \mathrm{x}=7.9 \\
\text { Corresponding lattice vectors } \\
\text { 3-Me07: } \\
\text { 1.000 0.000 } 0.000=8.066 \AA \\
\text { 3-Me08: } \\
\text { 1.000 0.000 - } 1.000=8.279 \AA \\
\text { Symmetry codes for SC }(\mathbf{3 - M e 0 7 ~ - ~ 3 - M e 0 8 ) ~} \\
\text { 1555.1 - } 1555.1 \\
\text { 1455.1 - } 1456.1 \\
\text { 1655.1 - } 1654.1 \\
\text { 3256.1 - } 3277.1\end{array}$ & \\
\hline
\end{tabular}


Table S6. Comparison 23-Me01-10 / 23-Me01-10.

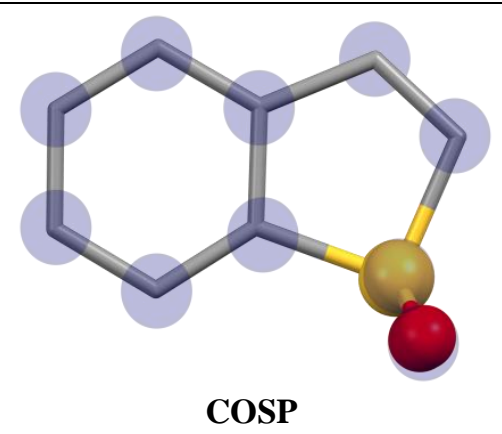

\begin{tabular}{|c|c|c|}
\hline \multirow{2}{*}{$\begin{array}{r}\text { Comparison } \\
\text { 23-Me01 - 23-Me02 }\end{array}$} & Results & Description \\
\hline & 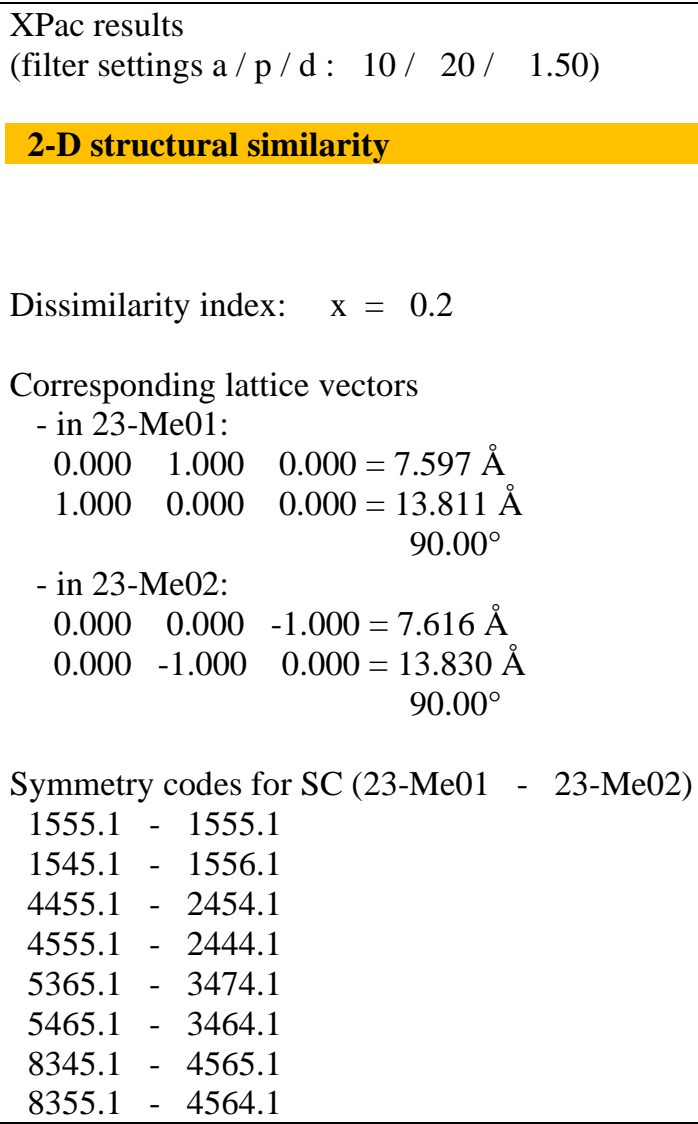 & Similarity D \\
\hline 23-Me01 - 23-Me03 & 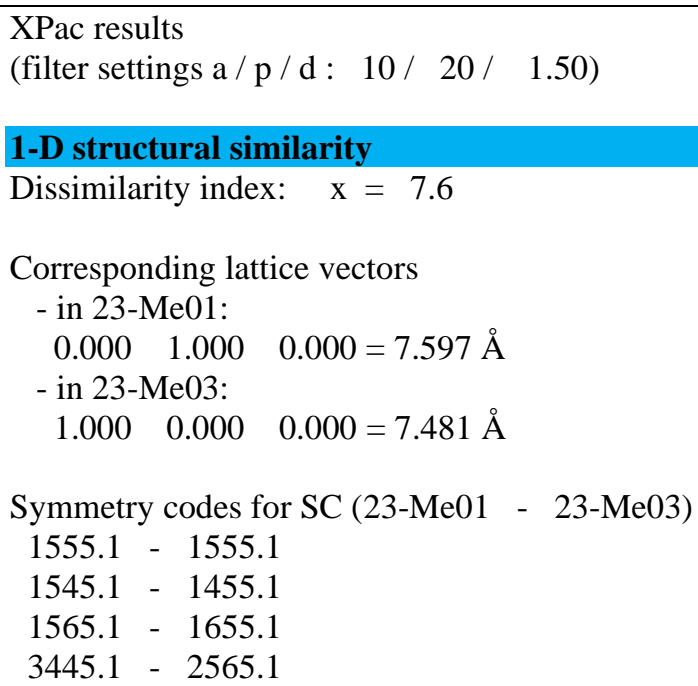 & do \\
\hline
\end{tabular}




\begin{tabular}{|c|c|c|}
\hline 23-Me01 - 23-Me04 & $\begin{array}{l}\text { XPac results } \\
\text { (filter settings a / p / }: 10 / 20 / 1.50) \\
\text { 1-D structural similarity } \\
\text { Dissimilarity index: } x=13.5 \\
\\
\text { Corresponding lattice vectors } \\
\text { - in 23-Me01: } \\
0.0001 .0000 .000=7.597 \AA \\
\text { - in } 23-\mathrm{Me} 04: \\
\text { 2.000 } 0.0000 .000=14.878 \AA \\
\text { Symmetry codes for SC }(23-\mathrm{Me} 01-23-\mathrm{Me} 04) \\
1555.1-1555.1 \\
1545.1-1455.1 \\
1565.1-1655.1 \\
3445.1-3364.1 \\
\end{array}$ & \\
\hline 23-Me01 - 23-Me06 & $\begin{array}{l}\text { XPac results } \\
\text { (filter settings a / } / \mathrm{d}: 10 / 20 / 1.50) \\
\text { 1-D structural similarity } \\
\text { Dissimilarity index: } \mathrm{x}=1.1 \\
\text { Stretch parameter: } \mathrm{D}=0.17 \AA \\
\text { For } \mathrm{n}=4 \text { neighbour(s) and } \mathrm{p}=12 \text { points } \\
\text { - delta[a] (angles) }=0.6^{\circ} \\
\text { - delta[p] (planes) }=0.8^{\circ} \\
\text { Corresponding lattice vectors } \\
\text { - in } 23-\mathrm{Me} 01: \\
0.0001 .0000 .000=7.597 \AA \\
\text { - in } 23-\mathrm{Me} 06: \\
1.0000 .0000 .000=7.411 \AA \\
\text { Symmetry codes for } \mathrm{SC}(23-\mathrm{Me} 01-23-\mathrm{Me} 06) \\
1555.1-1555.1 \\
1545.1-1455.1 \\
1565.1-1655.1 \\
3445.1-4466.1 \\
3455.1-4566.1\end{array}$ & 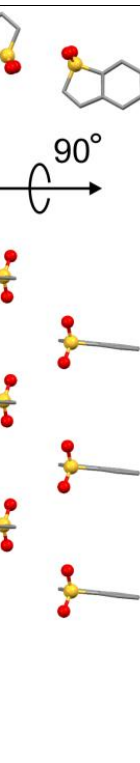 \\
\hline 23-Me01 - 23-Me07 & 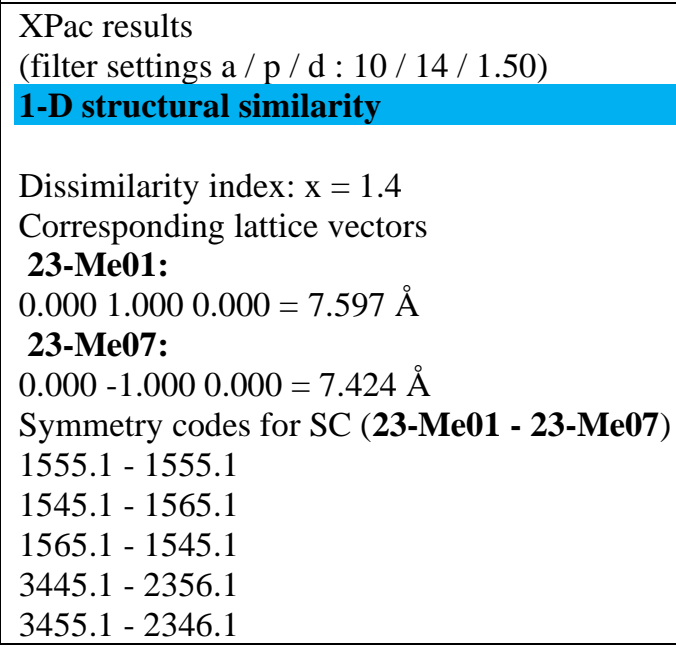 & $C^{90}$ \\
\hline
\end{tabular}




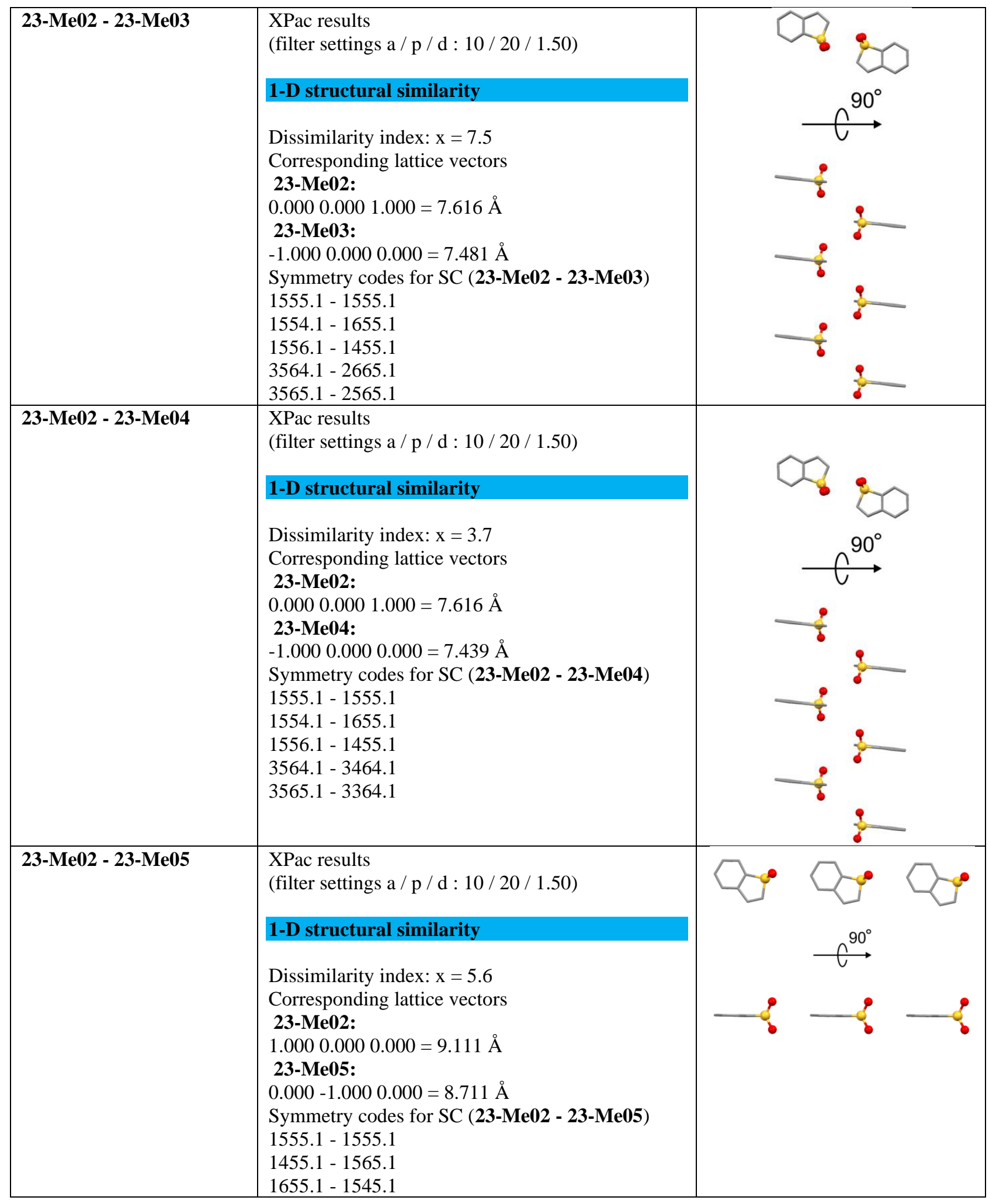




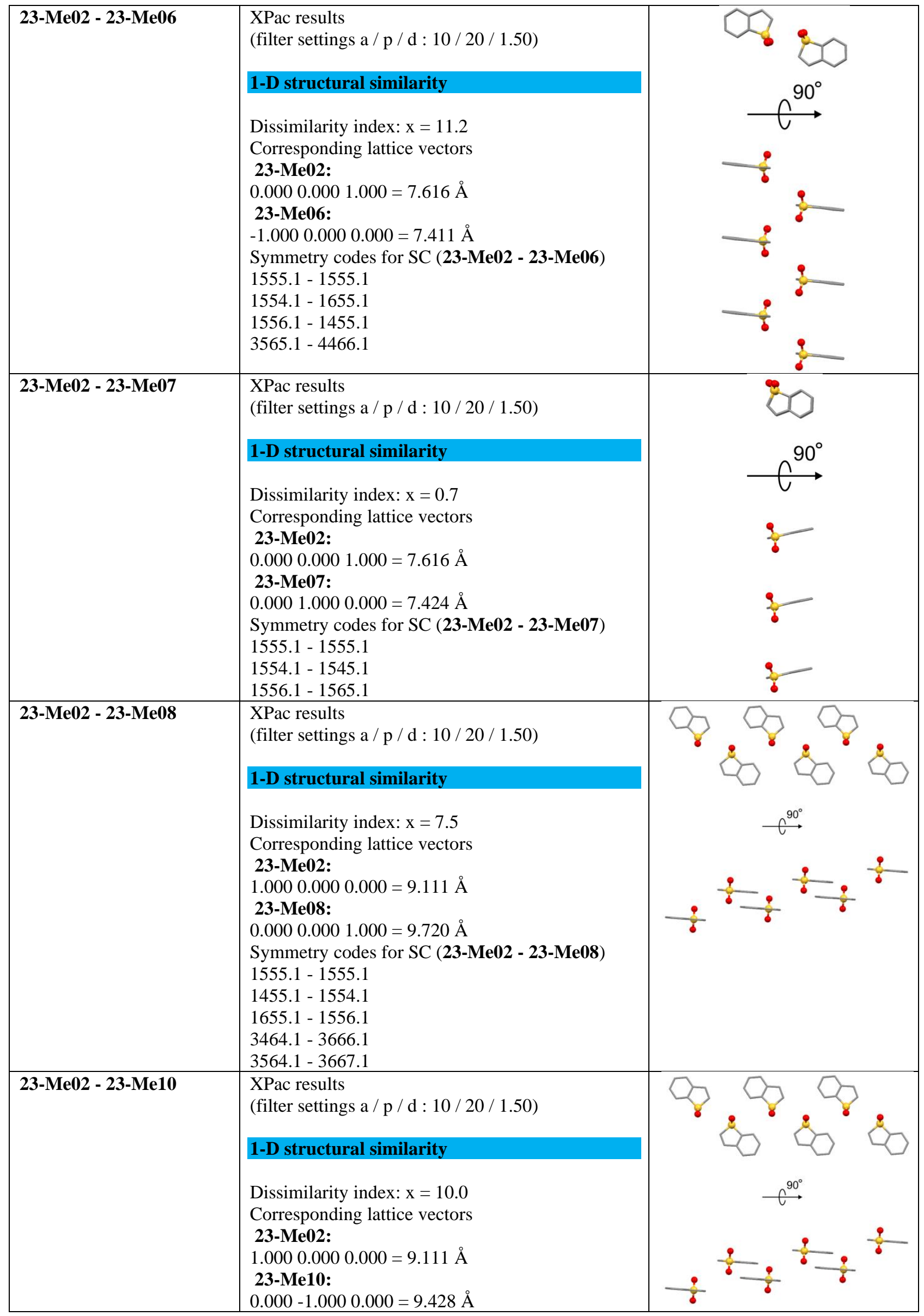




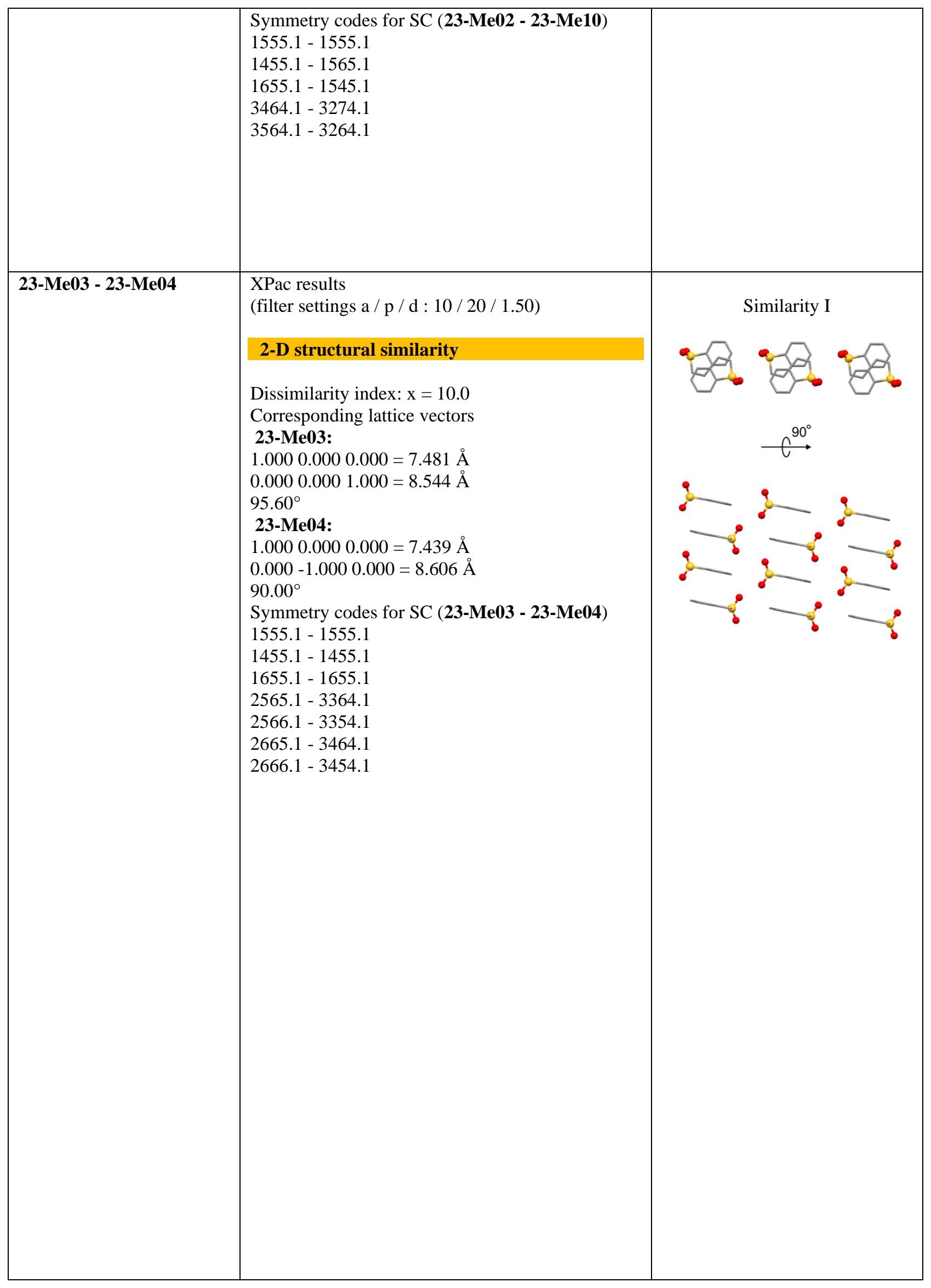




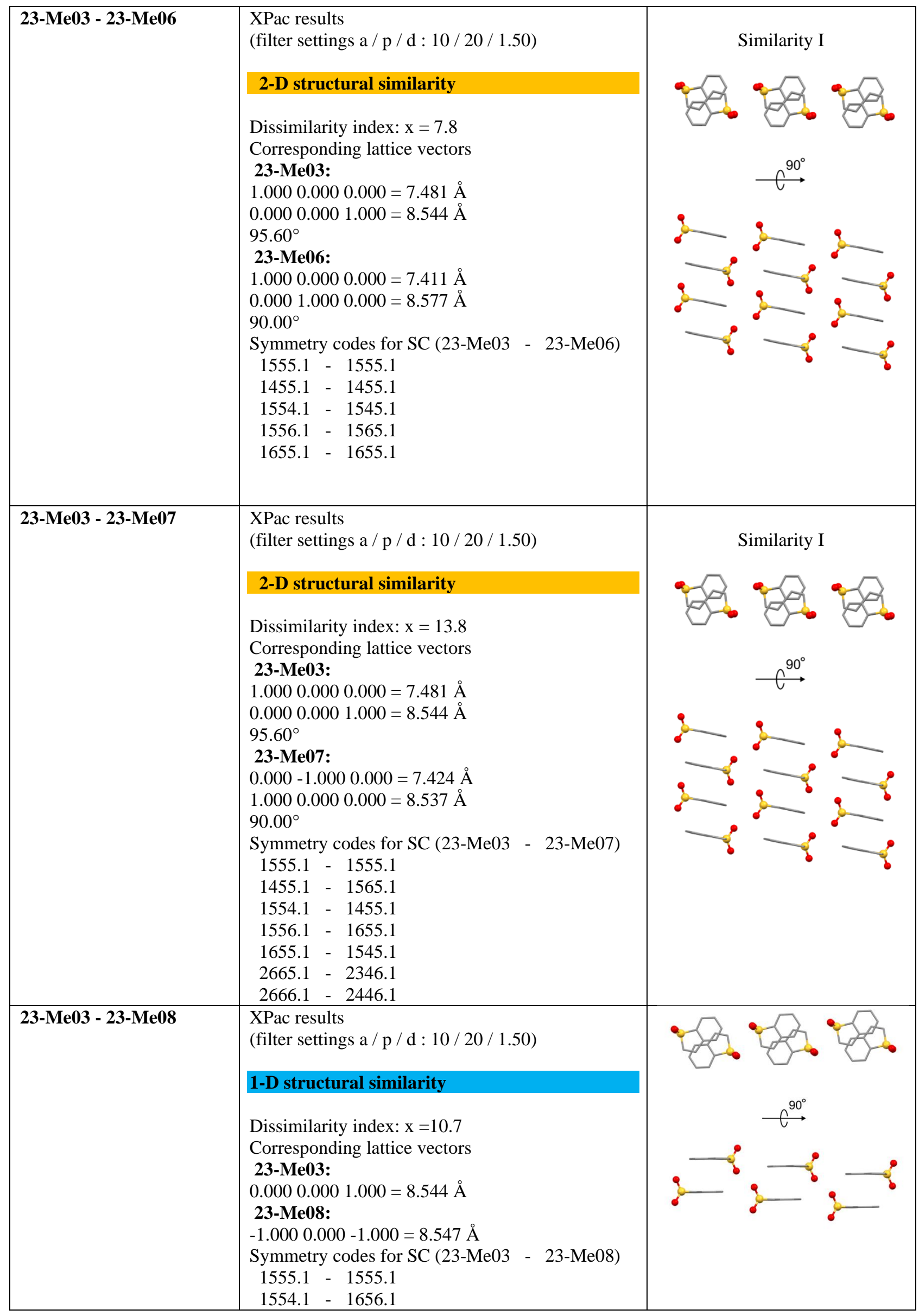




\begin{tabular}{|c|c|c|}
\hline & $\begin{array}{l}1556.1-1454.1 \\
2665.1-3667.1 \\
2666.1-3566.1\end{array}$ & \\
\hline 23-Me04 - 23-Me05 & $\begin{array}{l}\text { XPac results } \\
\text { (filter settings a / p / d : } 10 \text { / } 20 \text { / 1.50) } \\
\text { 1-D structural similarity } \\
\text { Dissimilarity index: } x=6.7 \\
\text { Corresponding lattice vectors } \\
\text { 23-Me04: } \\
\text { 1.000 0.000 } 0.000=7.439 \AA \\
\mathbf{2 3 - M e 0 5 :} \\
\text { 1.000 0.000 } 0.000=7.595 \AA \\
\text { Symmetry codes for SC (23-Me04 - 23-Me05) } \\
\text { 1555.1 - } 1555.1 \\
3354.1 \text { - } 3365.1 \\
3454.1-3465.1\end{array}$ & Similarity S2 \\
\hline 23-Мe04 - 23-Мe06 & $\begin{array}{l}\text { XPac results } \\
\text { (filter settings a / p / } \mathrm{d}: 10 \text { / } 20 \text { / 1.50) } \\
\text { 2-D structural similarity } \\
\text { Dissimilarity index: } \mathrm{x}=14.4 \\
\text { Corresponding lattice vectors } \\
\mathbf{2 3 - M e 0 4 :} \\
1.0000 .0000 .000=7.439 \AA \\
0.0001 .0000 .000=8.606 \AA \\
90.00^{\circ} \\
\mathbf{2 3 - M e 0 6 :} \\
1.0000 .0000 .000=7.411 \AA \\
0.000-1.0000 .000=8.577 \AA \\
90.00^{\circ} \\
\text { Symmetry codes for SC }(23-\mathrm{Me} 04-23-\mathrm{Me} 06) \\
1555.1-1555.1 \\
1455.1-1455.1 \\
1545.1-1565.1 \\
1565.1-1545.1 \\
1655.1-1655.1 \\
3354.1-4476.1 \\
3364.1-4466.1\end{array}$ & Simila \\
\hline
\end{tabular}




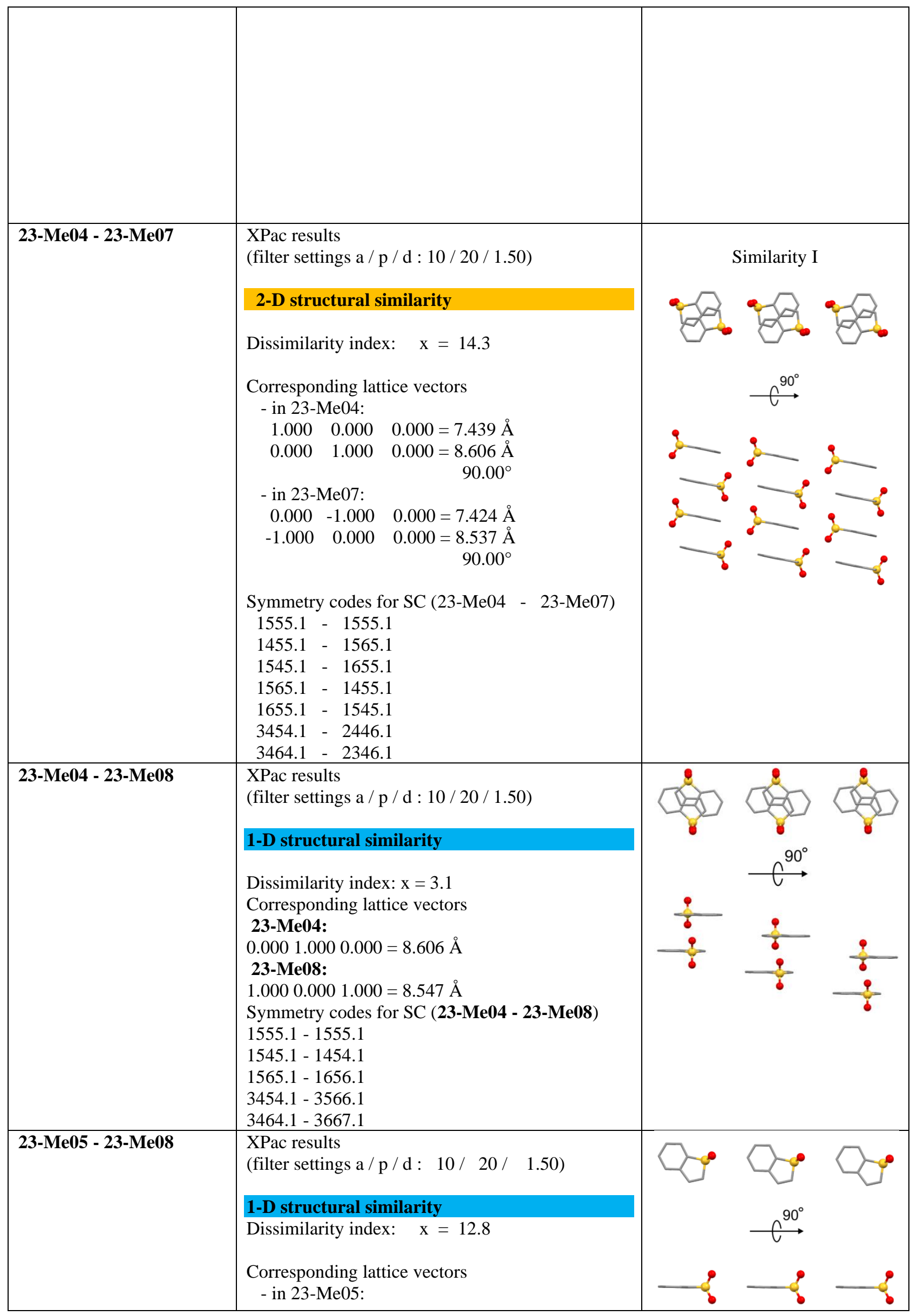




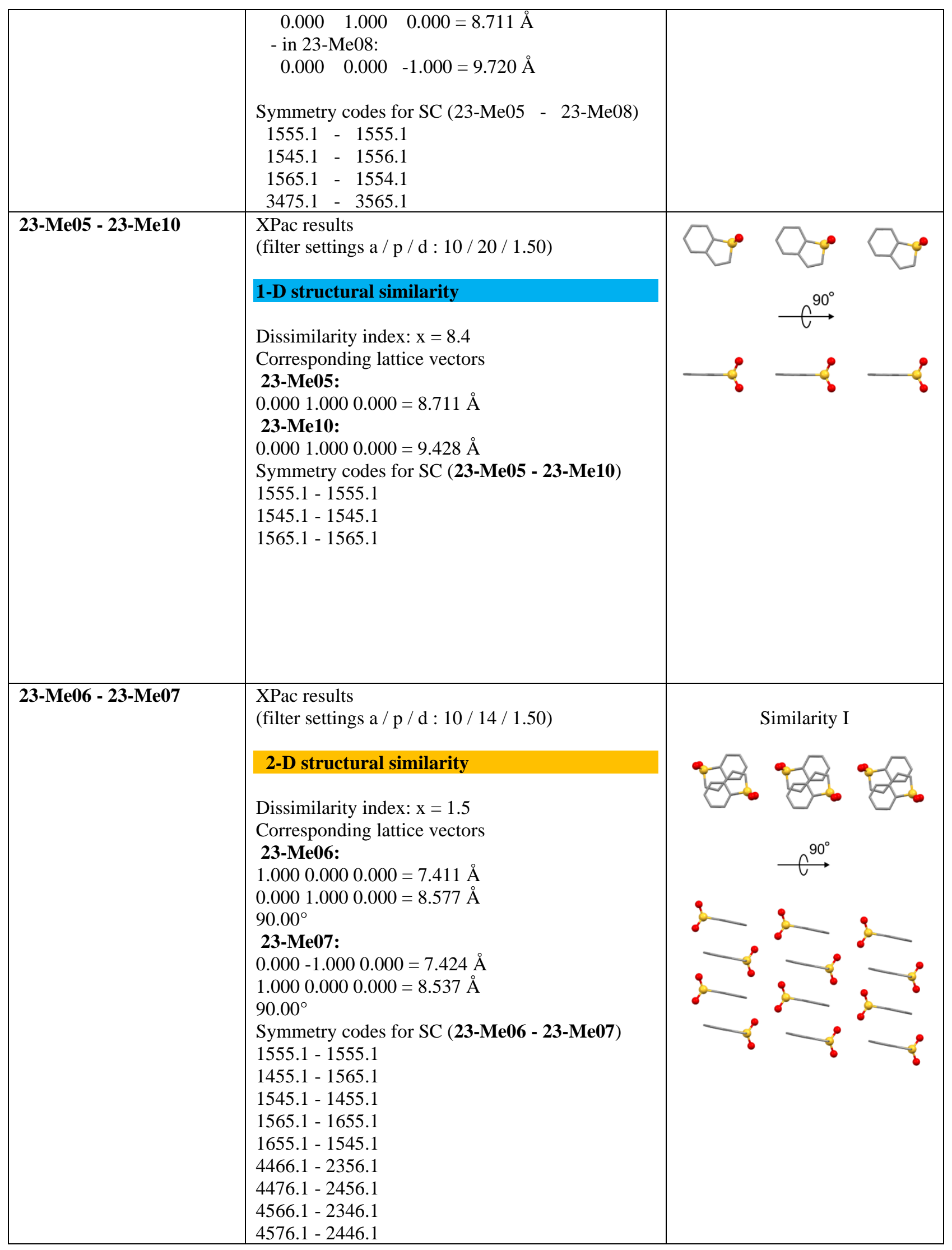




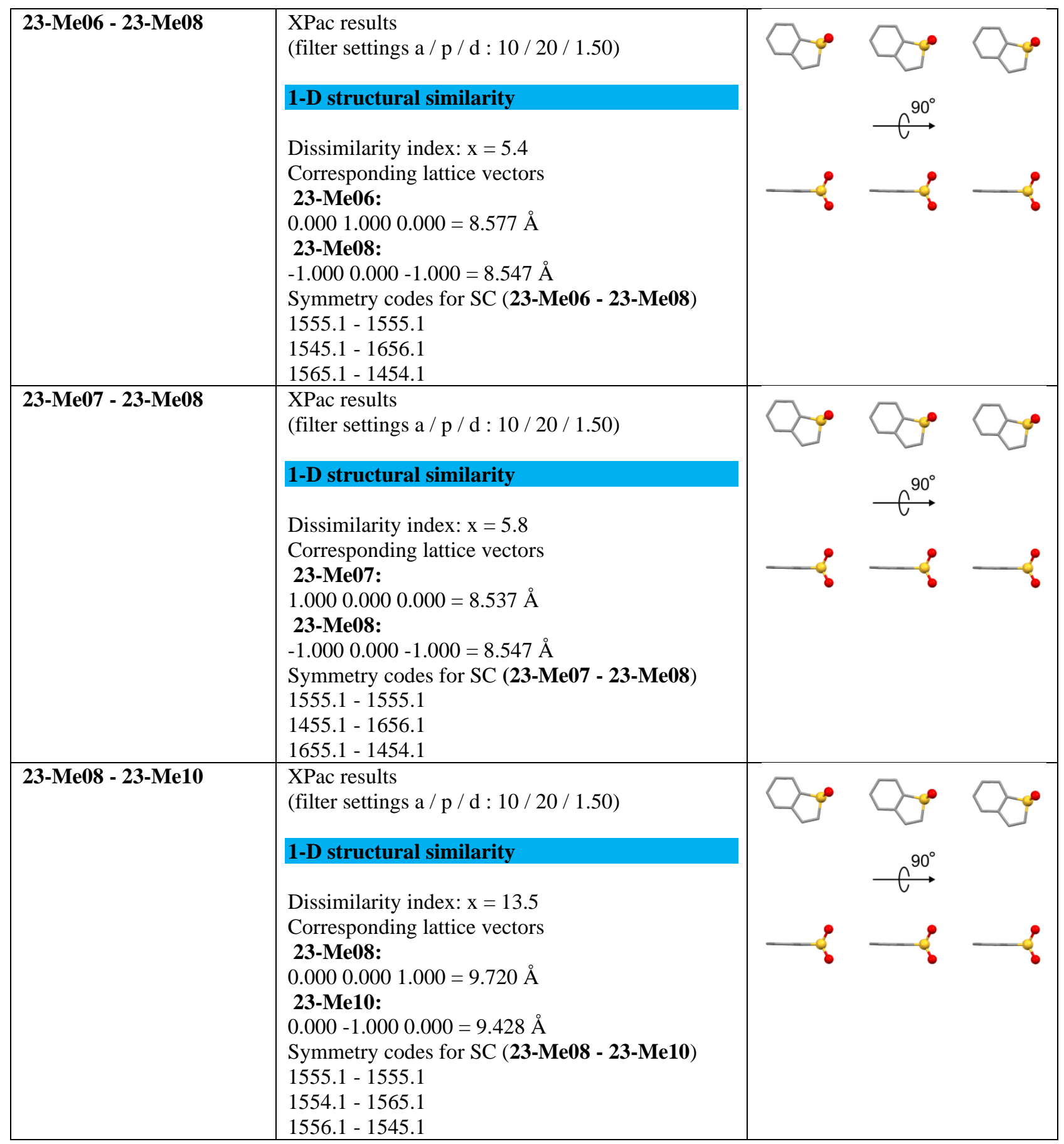




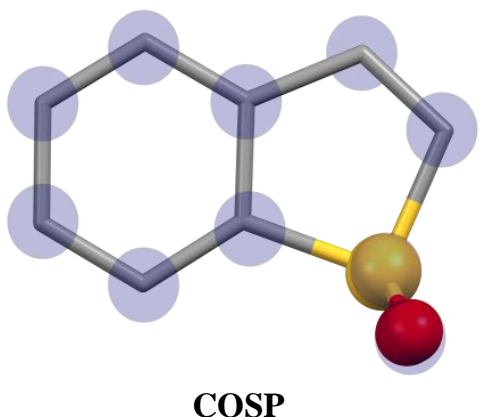

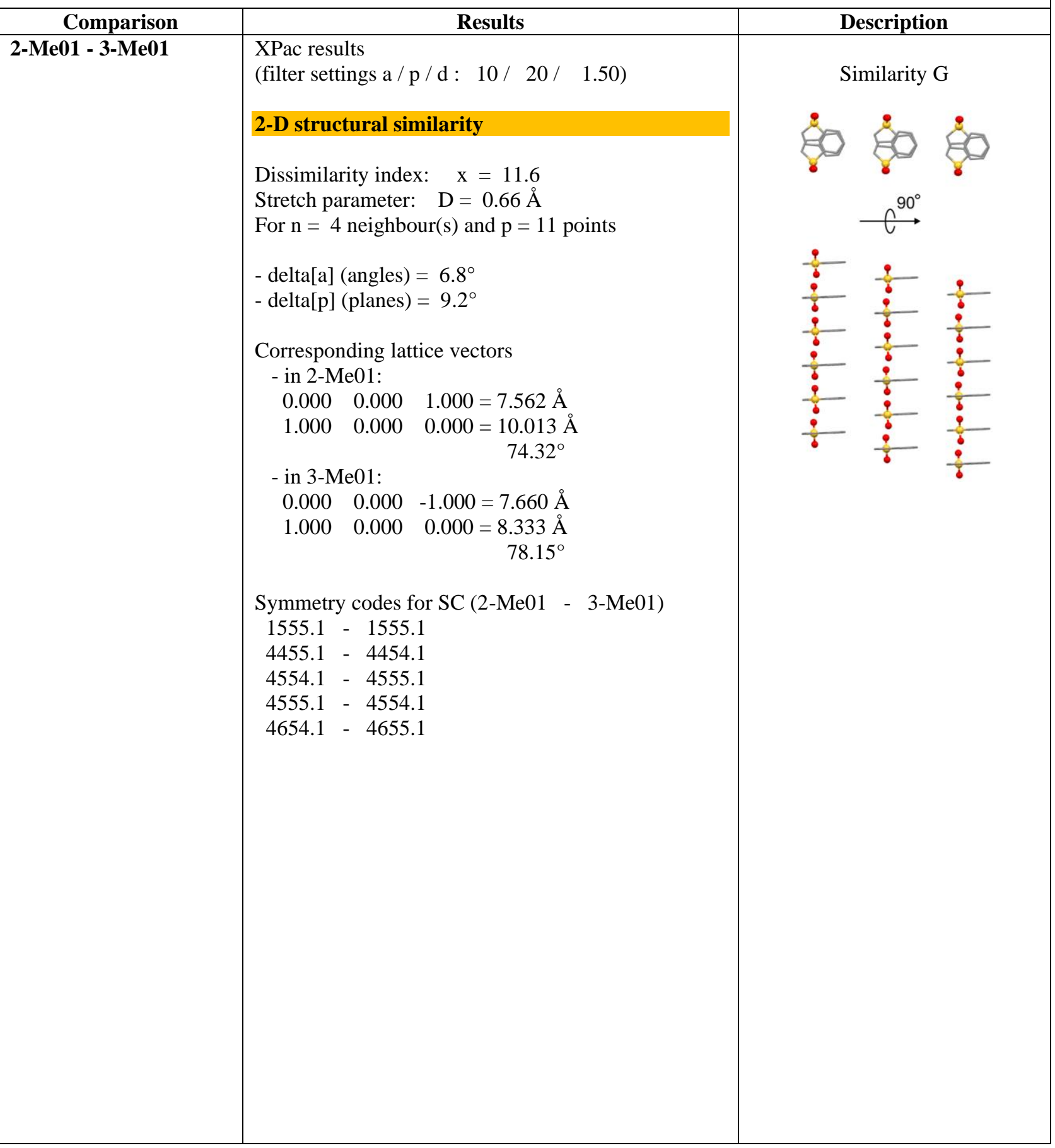




\begin{tabular}{|c|c|c|}
\hline 2-Me01 - 3-Me03 & 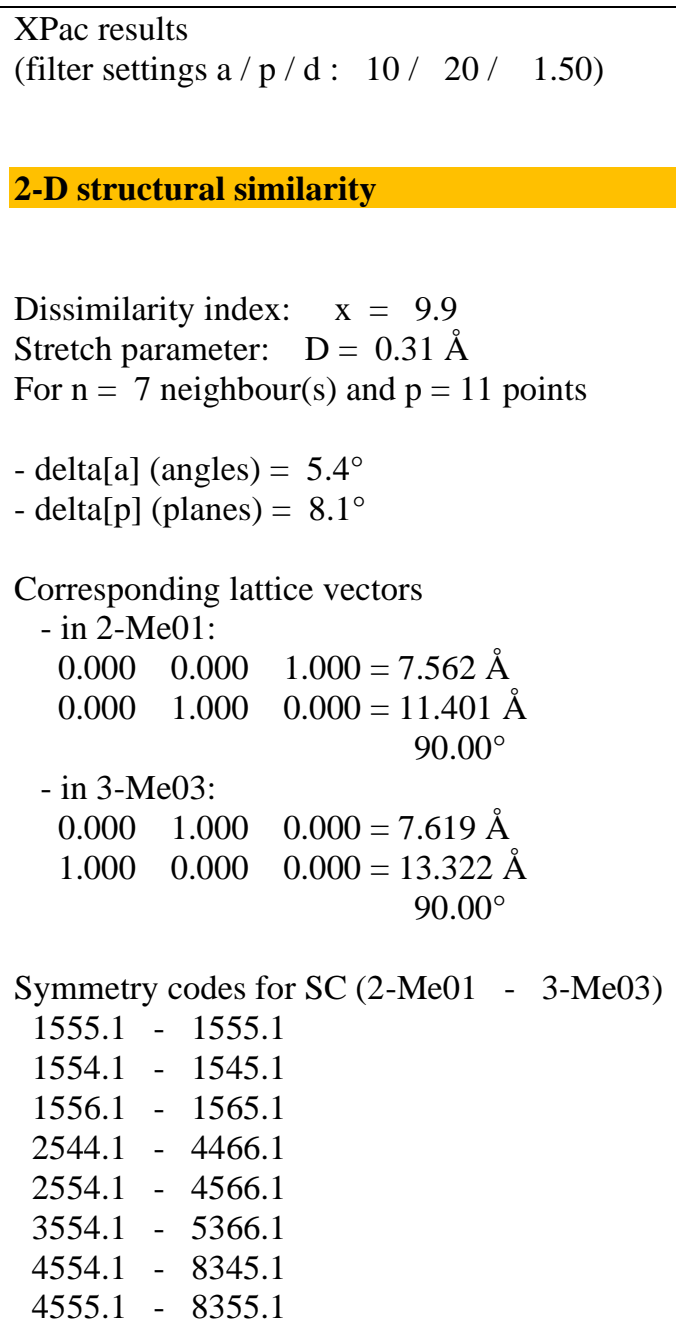 & Similarity D \\
\hline 2-Me01 - 3-Me05 & 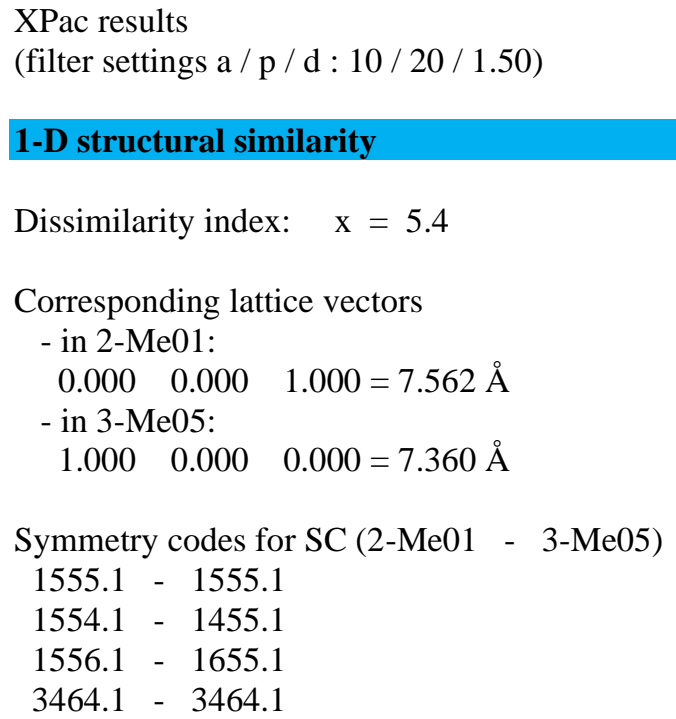 & $\overbrace{\frac{2}{8}}^{\stackrel{9}{90^{\circ}}}$ \\
\hline
\end{tabular}




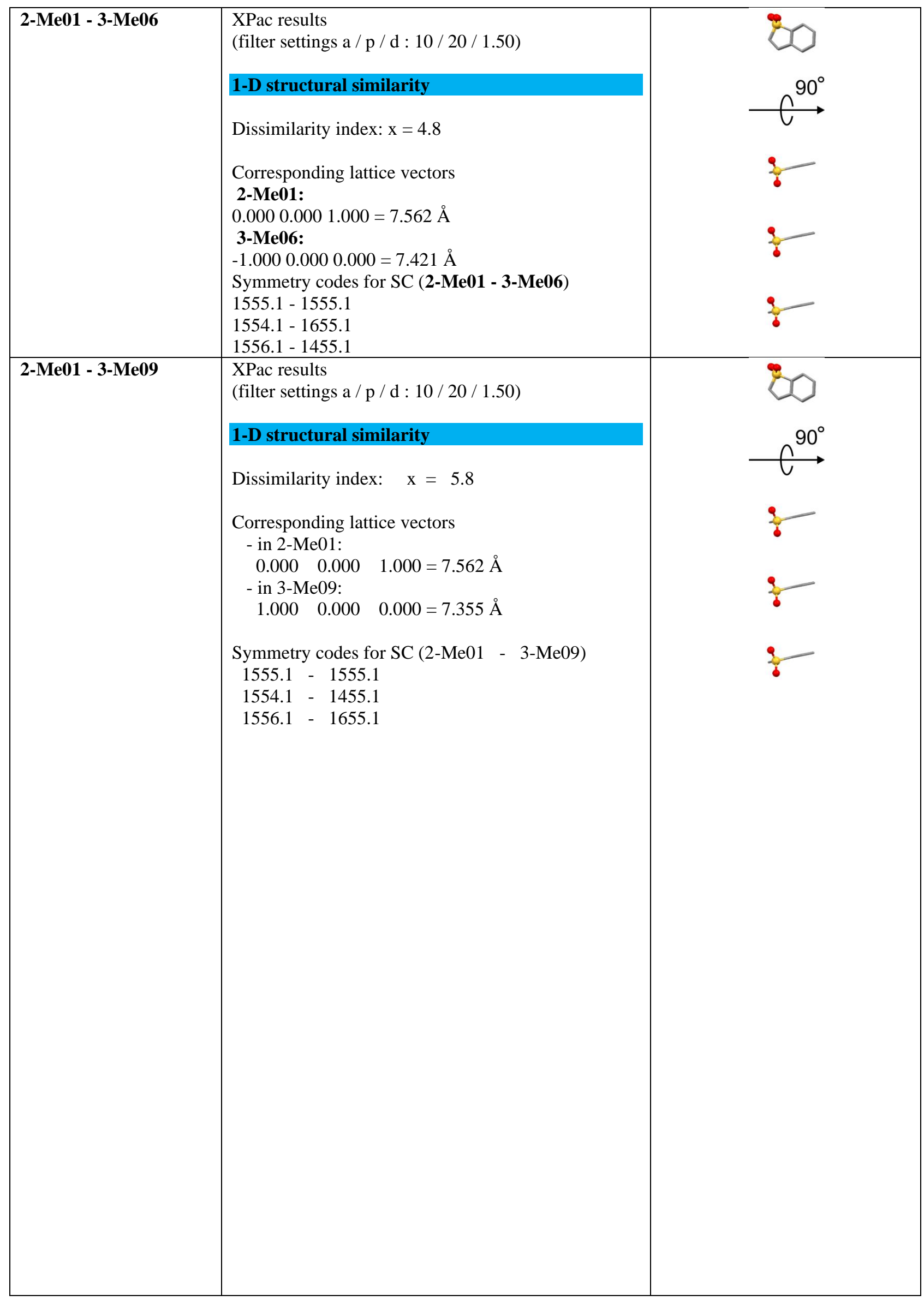




\begin{tabular}{|c|c|c|}
\hline 2-Me02 - 3-Me01 & 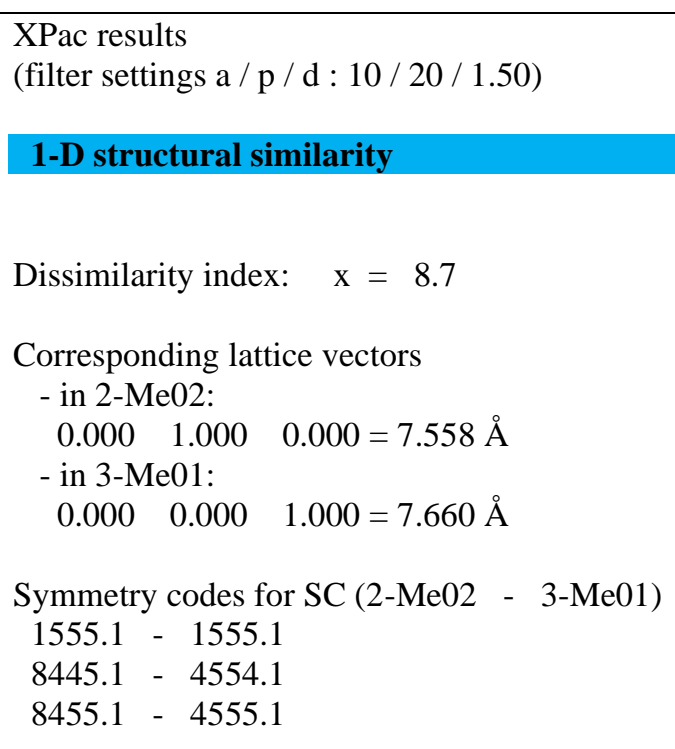 & Similarity S1 \\
\hline 2-Me02 - 3-Me03 & 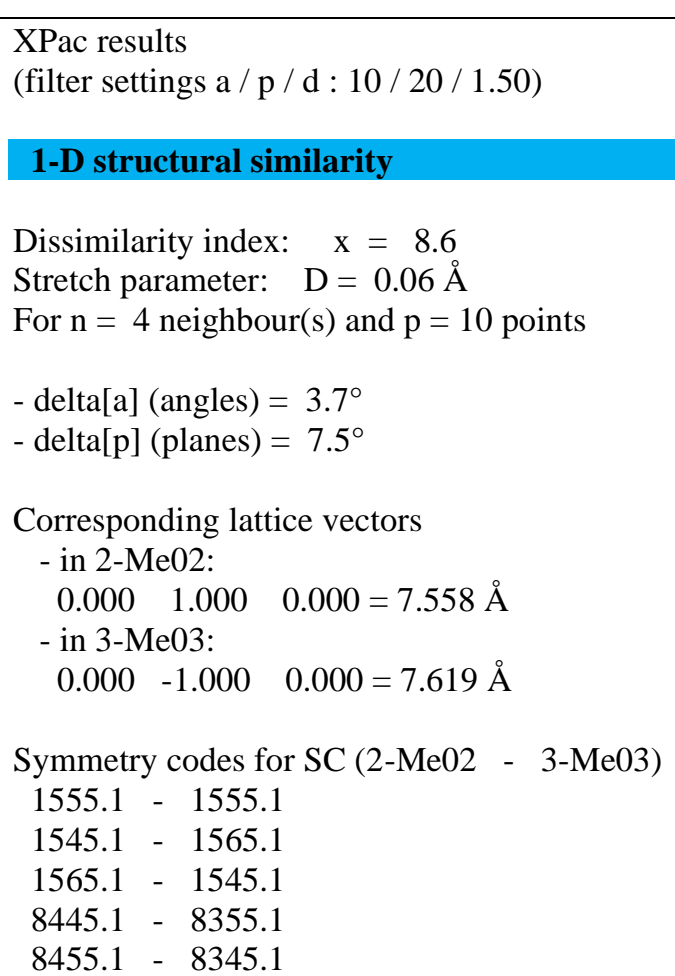 & Similarity S1 \\
\hline 2-Me02 - 3-Me06 & $\begin{array}{l}\text { XPac results } \\
\text { (filter settings a / p / d : } 10 \text { / } 20 \text { / 1.50) } \\
\text { 1-D structural similarity } \\
\text { Dissimilarity index: } \mathrm{x}=11.2 \\
\text { Corresponding lattice vectors } \\
\text { 2-Me02: } \\
0.0001 .0000 .000=7.558 \AA \\
\text { 3-Me06: } \\
\text { 1.000 0.000 } 0.000=7.421 \AA \\
\text { Symmetry codes for SC (2-Me02 - 3-Me06) } \\
\text { 1555.1 - } 1555.1 \\
1545.1 \text { - } 1455.1 \\
1565.1 \text { - } 1655.1\end{array}$ & \% \\
\hline
\end{tabular}




\begin{tabular}{|c|c|c|}
\hline 2-Me02 - 3-Me09 & 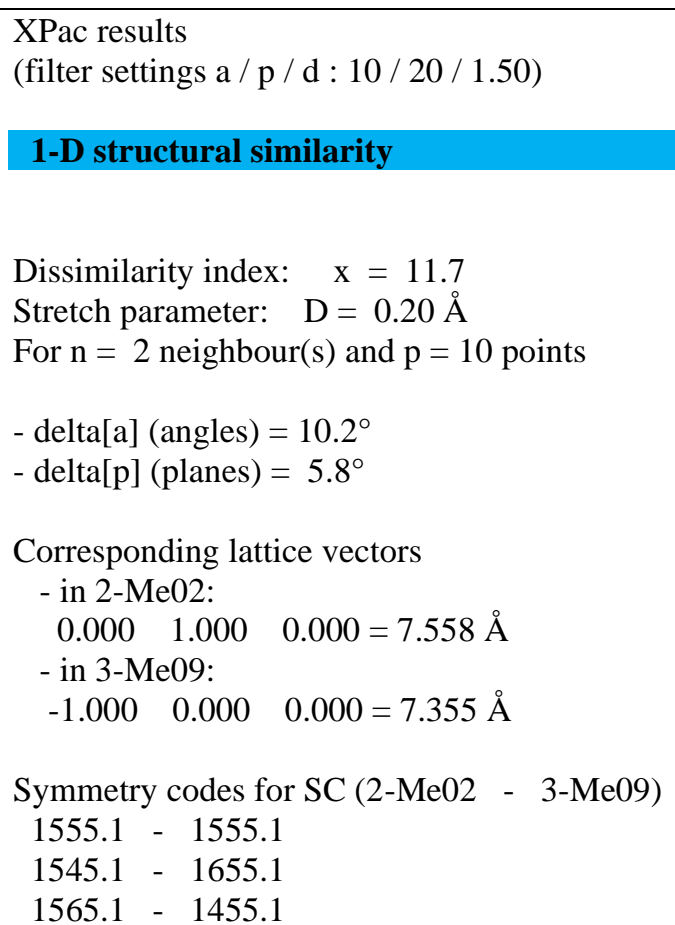 & \\
\hline 2-Me05 - 3-Me03 & 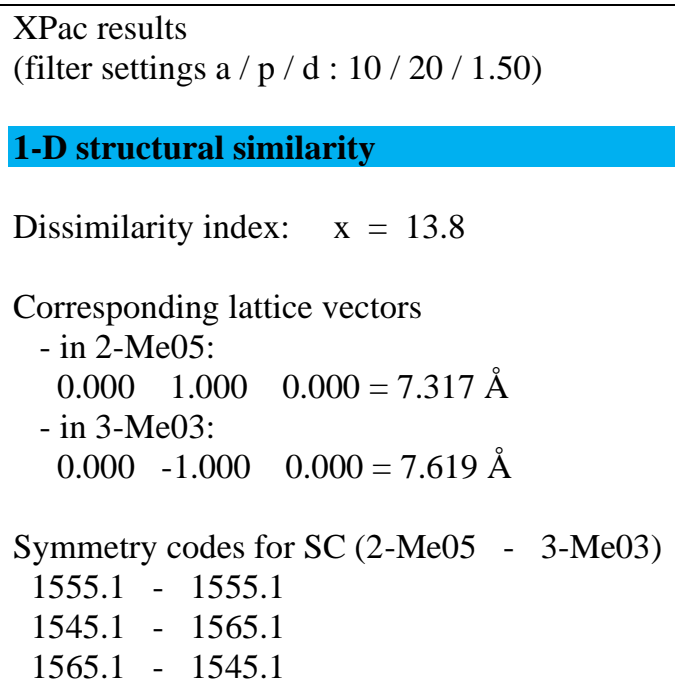 & 8. \\
\hline
\end{tabular}




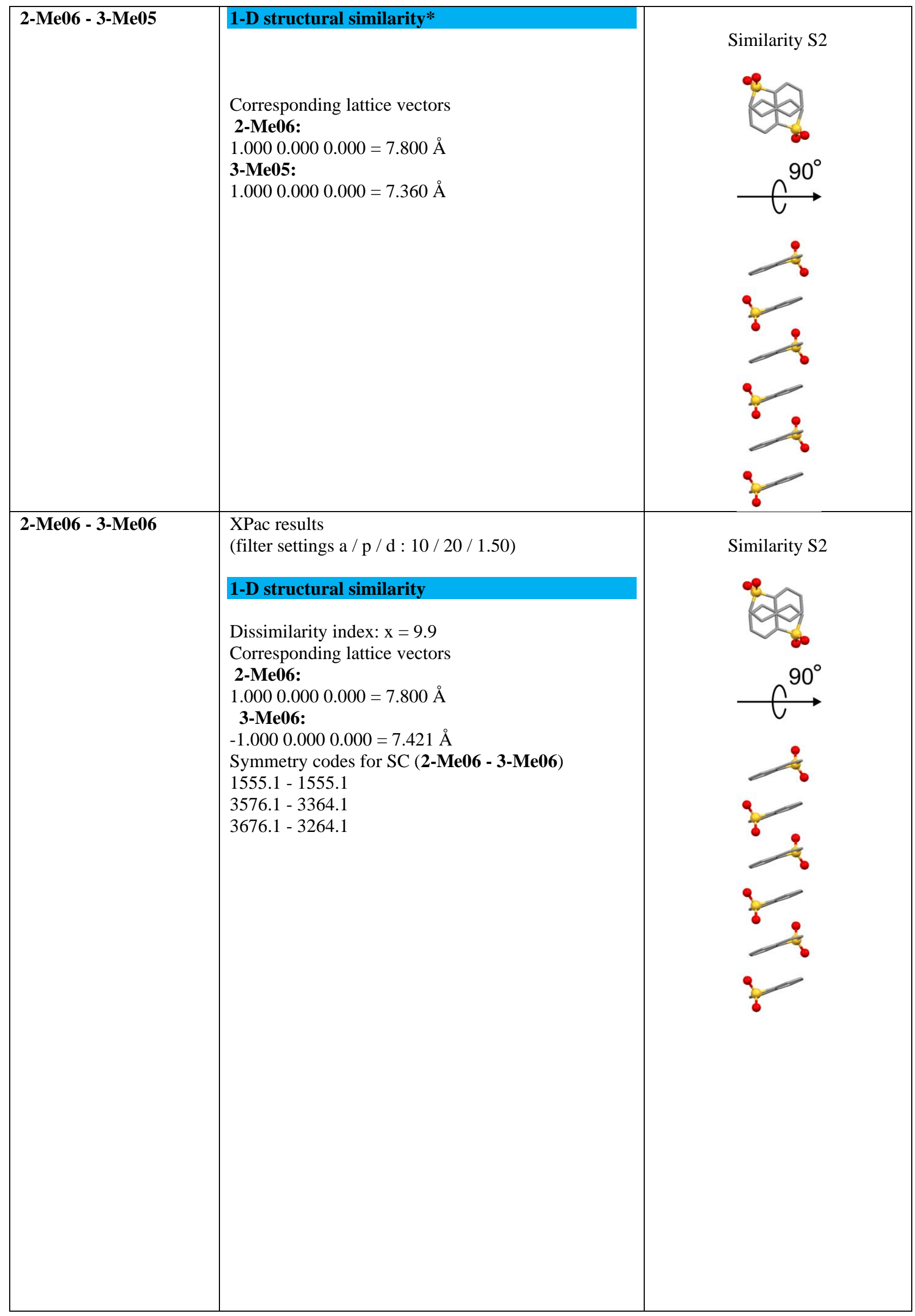




\begin{tabular}{|c|c|c|}
\hline 2-Me06 - 3-Me08 & $\begin{array}{l}\text { XPac results } \\
\text { (filter settings a / p / d : } 10 \text { / } 20 \text { / 1.50) } \\
\text { 1-D structural similarity } \\
\text { Dissimilarity index: } \mathrm{x}=7.1 \\
\text { Corresponding lattice vectors } \\
\text { 2-Me06: } \\
\text { 1.000 0.000 } 0.000=7.800 \AA \\
\text { 3-Me08: } \\
-1.0000 .0000 .000=7.762 \AA \\
\text { Symmetry codes for SC (2-Me06 - 3-Me08) } \\
1555.1 \text { - } 1555.1 \\
3576.1 \text { - } 3277.1 \\
3676.1-3177.1\end{array}$ & Similarity S2 \\
\hline 2-Me06 - 3-Me10 & 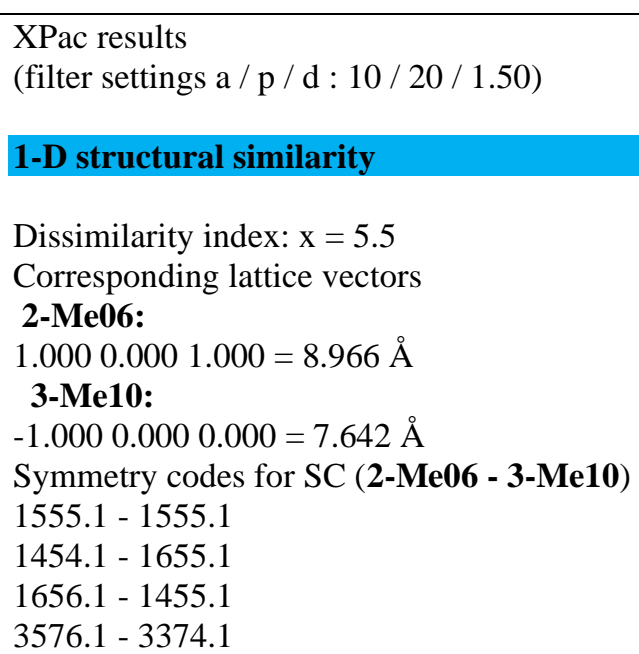 & b \\
\hline 2-Me10 - 3-Me01 & $\begin{array}{l}\text { XPac results } \\
\text { (filter settings a / p / d : } 10 \text { / } 20 \text { / 1.50) } \\
\text { 1-D structural similarity } \\
\text { Dissimilarity index: } \mathrm{x}=6.7 \\
\text { Corresponding lattice vectors } \\
\text { 2-Me10: } \\
\text { 1.000 0.000 }-1.000=9.051 \AA \\
\text { 3-Me01: } \\
-1.0000 .0000 .000=8.333 \AA \\
\text { Symmetry codes for SC (2-Me10 - 3-Me01) } \\
1555.1 \text { - } 1555.1 \\
\text { 1456.1 - } 1655.1 \\
1654.1-1455.1\end{array}$ & 5 \\
\hline
\end{tabular}




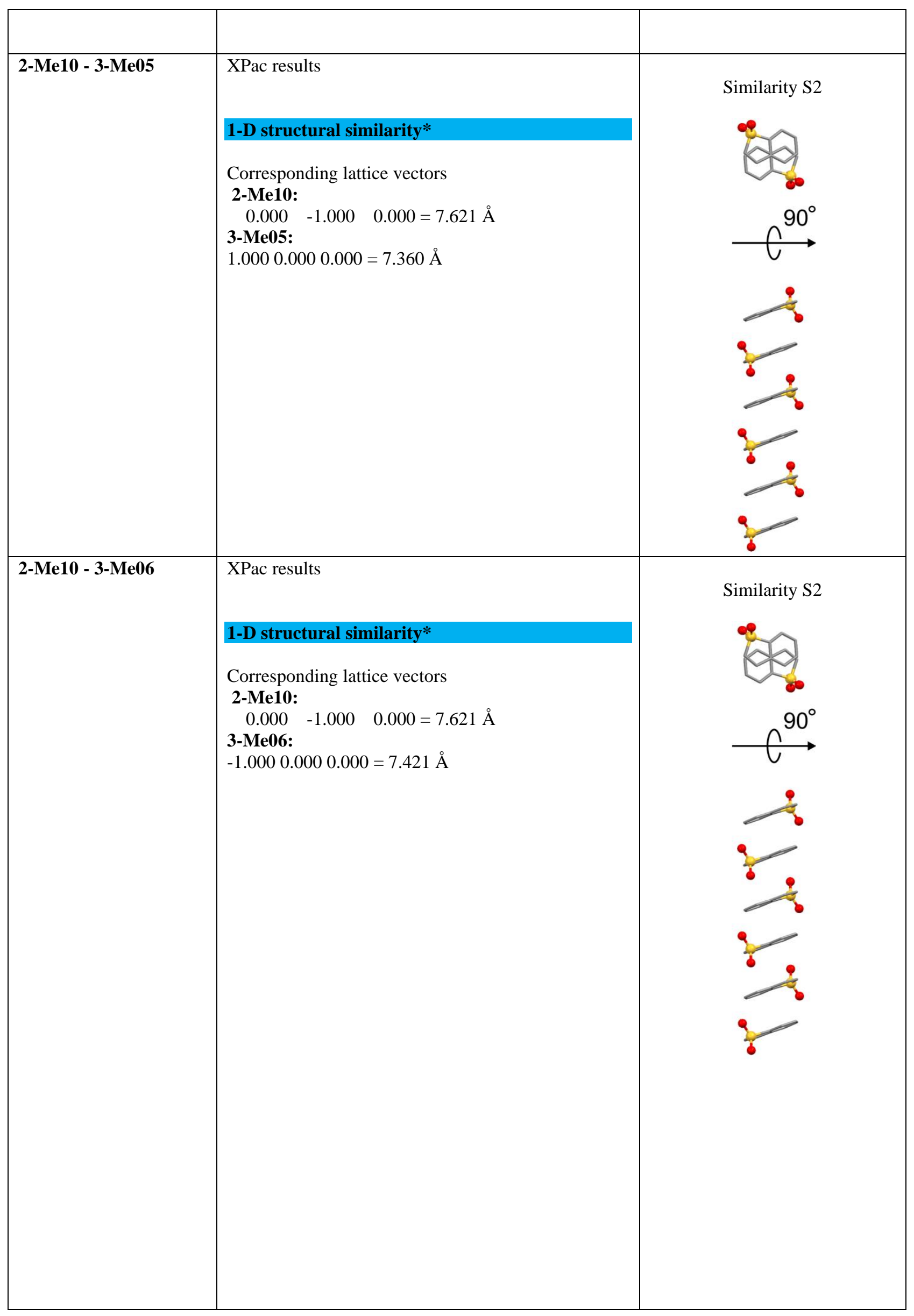




\begin{tabular}{|l|l|l|}
\hline 2-Me10 - 3-Me08 & XPac results & \\
1-D structural similarity* & Similarity S2 \\
Corresponding lattice vectors & 2-Me10: \\
$0.000 \quad-1.000 \quad 0.000=7.621 \AA$ \\
3-Me08: \\
$-1.0000 .0000 .000=7.762 \AA$
\end{tabular}


Table S8. Comparison 23-Me_01-10 / 2-Me_01-10.

\begin{tabular}{|c|c|c|}
\hline & COSP & \\
\hline 23-Me01 - 2-Me01 & $\begin{array}{l}\text { XPac results } \\
\text { (filter settings a / p / d : } 10 / 20 / 1.50) \\
\text { 2-D structural similarity } \\
\text { Dissimilarity index: } x=10.5 \\
\text { Corresponding lattice vectors } \\
\text { 23-Me01: } \\
0.0001 .0000 .000=7.597 \AA \\
1.0000 .0000 .000=13.811 \AA \\
90.00^{\circ} \\
\text { 2-Me01: } \\
0.0000 .000-1.000=7.562 \AA \\
0.0001 .0000 .000=11.401 \AA \\
90.00^{\circ} \\
\text { Symmetry codes for SC (23-Me01 - 2- } \\
\text { Me01) } \\
1555.1 \text { - } 1555.1 \\
1545.1 \text { - } 1556.1 \\
1565.1 \text { - } 1554.1 \\
4455.1 \text { - } 2544.1 \\
4555.1 \text { - } 2554.1 \\
5365.1 \text { - } 3554.1 \\
5465.1 \text { - } 3564.1\end{array}$ & Similarity D \\
\hline 23-Me01 - 2-Me02 & 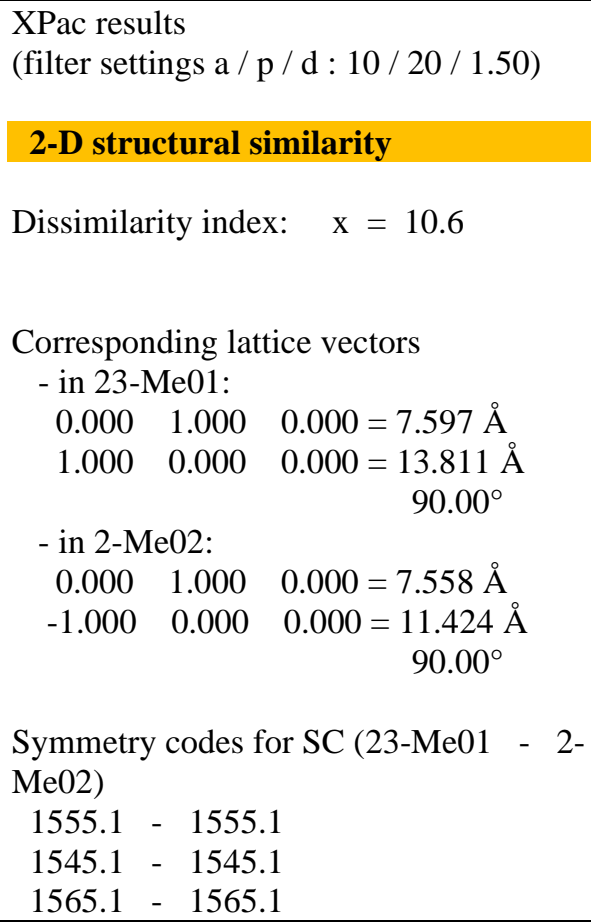 & $\uparrow^{90^{\circ}}$ \\
\hline
\end{tabular}




\begin{tabular}{|c|c|c|}
\hline & $\begin{array}{l}3445.1-3445.1 \\
3455.1-3455.1 \\
6455.1-6555.1 \\
6555.1-6455.1 \\
8345.1-8445.1 \\
8355.1-8455.1\end{array}$ & \\
\hline 23-Me01 - 2-Me05 & 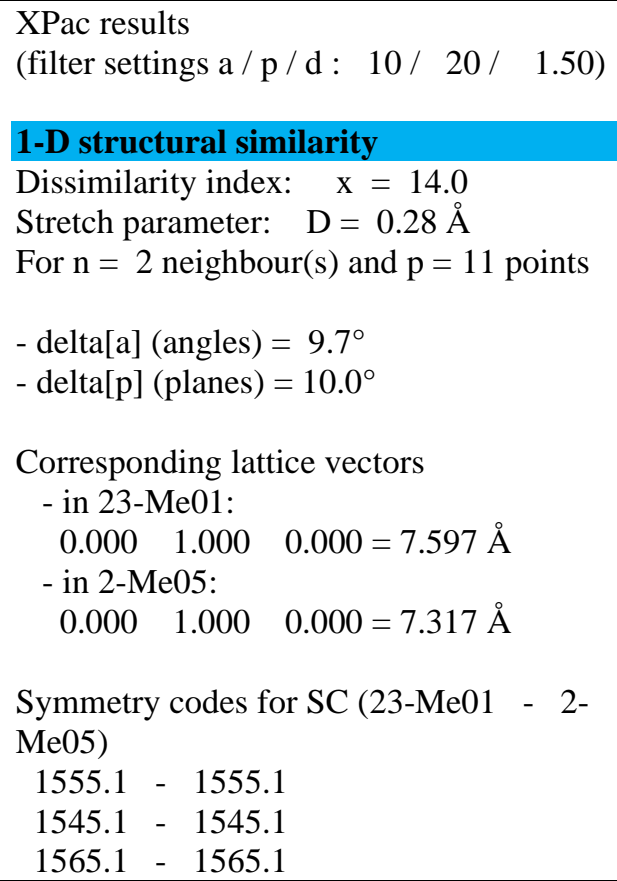 & b \\
\hline 23-Me01 - 2-Me08 & 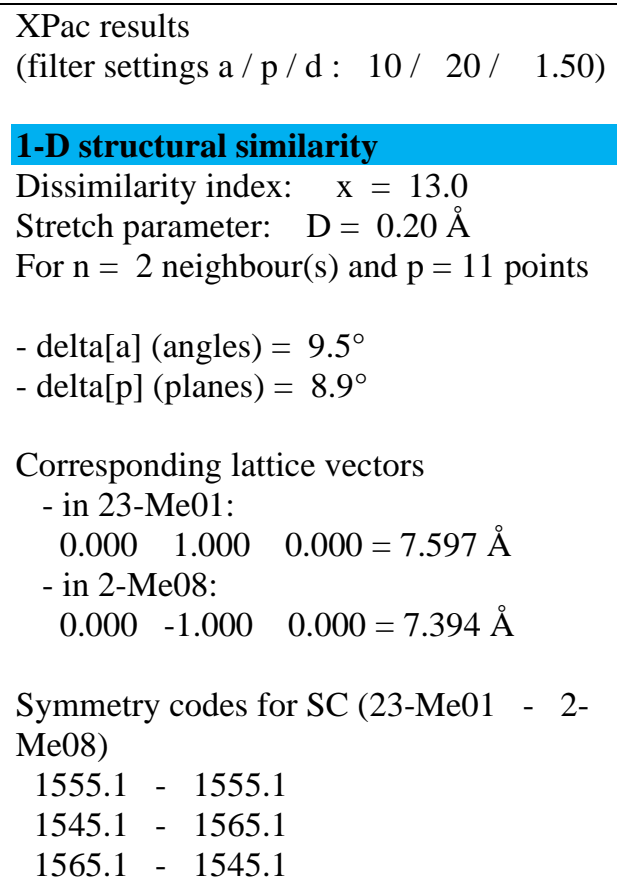 & 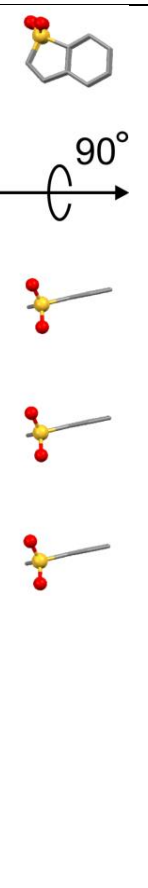 \\
\hline 23-Me02 - 2-Me01 & XPac results & \\
\hline
\end{tabular}




\begin{tabular}{|c|c|c|}
\hline & $\begin{array}{l}\text { (filter settings a / } \mathrm{p} / \mathrm{d}: 10 / 20 / 4.00) \\
\text { 3-D structural similarity } \\
\text { Dissimilarity index: } \mathrm{x}=11.7 \\
\text { Corresponding lattice vectors } \\
\text { 23-Me02: } \\
0.0000 .0001 .000=7.616 \AA \\
0.0001 .0000 .000=13.830 \AA \\
90.00^{\circ} \\
\text { 2-Me01: } \\
0.0000 .0001 .000=7.562 \AA \\
0.000-1.0000 .000=11.401 \AA \\
90.00^{\circ} \\
\text { Symmetry codes for SC }(23-\mathrm{Me} 02-2- \\
\text { Me01) } \\
1555.1-1555.1 \\
1554.1-1554.1 \\
1556.1-1556.1 \\
2444.1-2554.1 \\
2454.1-2544.1 \\
3464.1-3564.1 \\
3474.1-3554.1\end{array}$ & \\
\hline 23-Me02 - 2-Me02 & 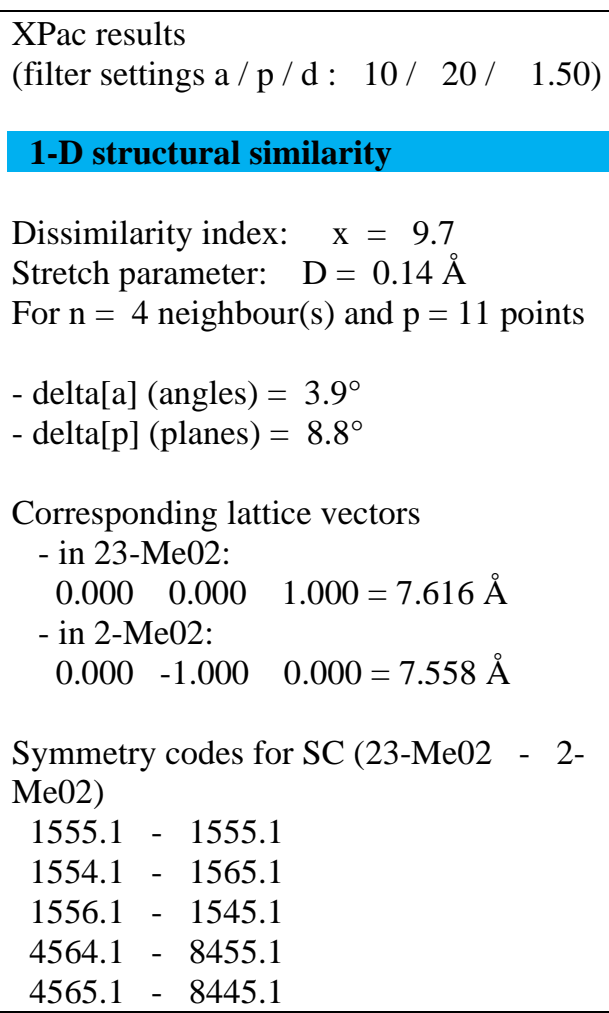 & Similarity S1 \\
\hline
\end{tabular}




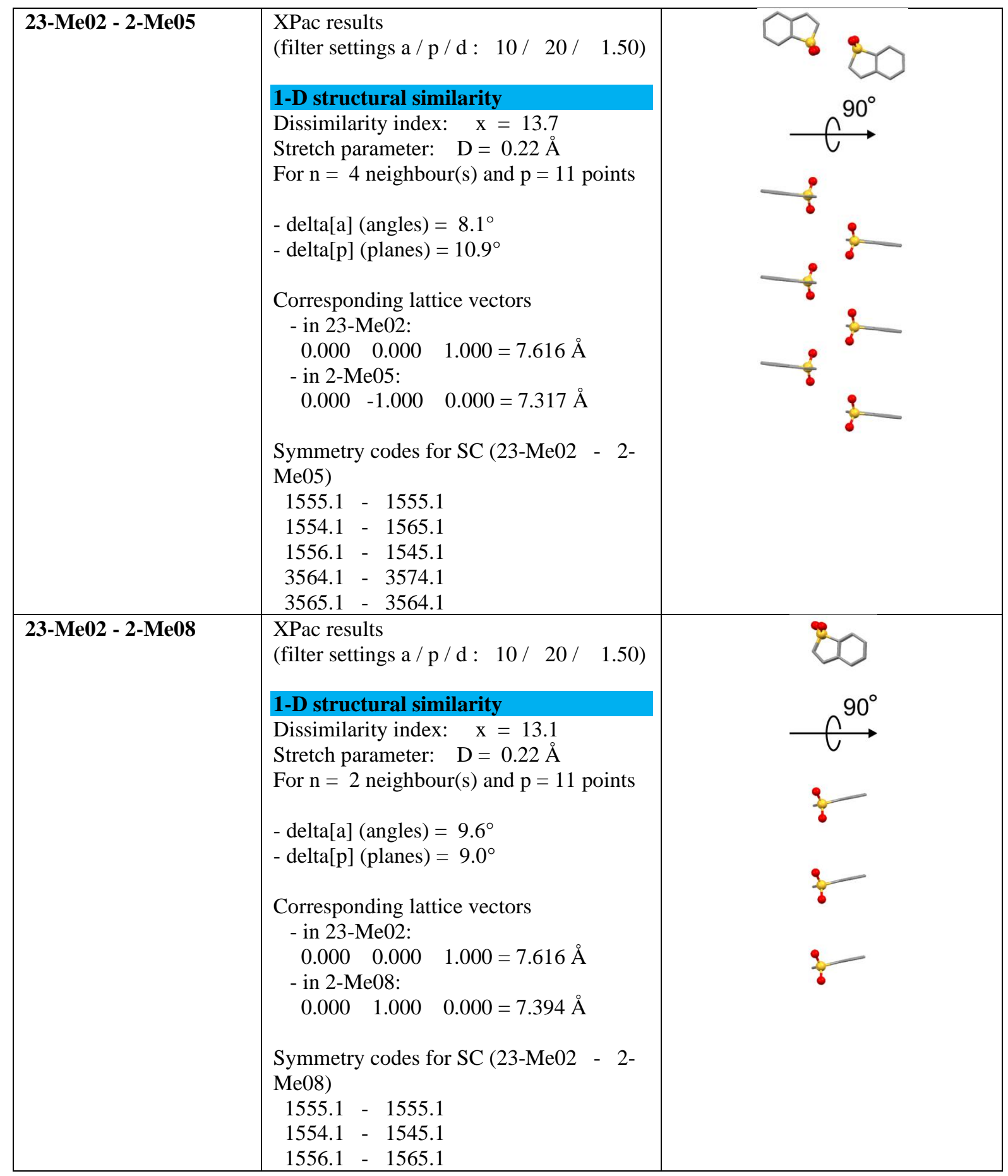




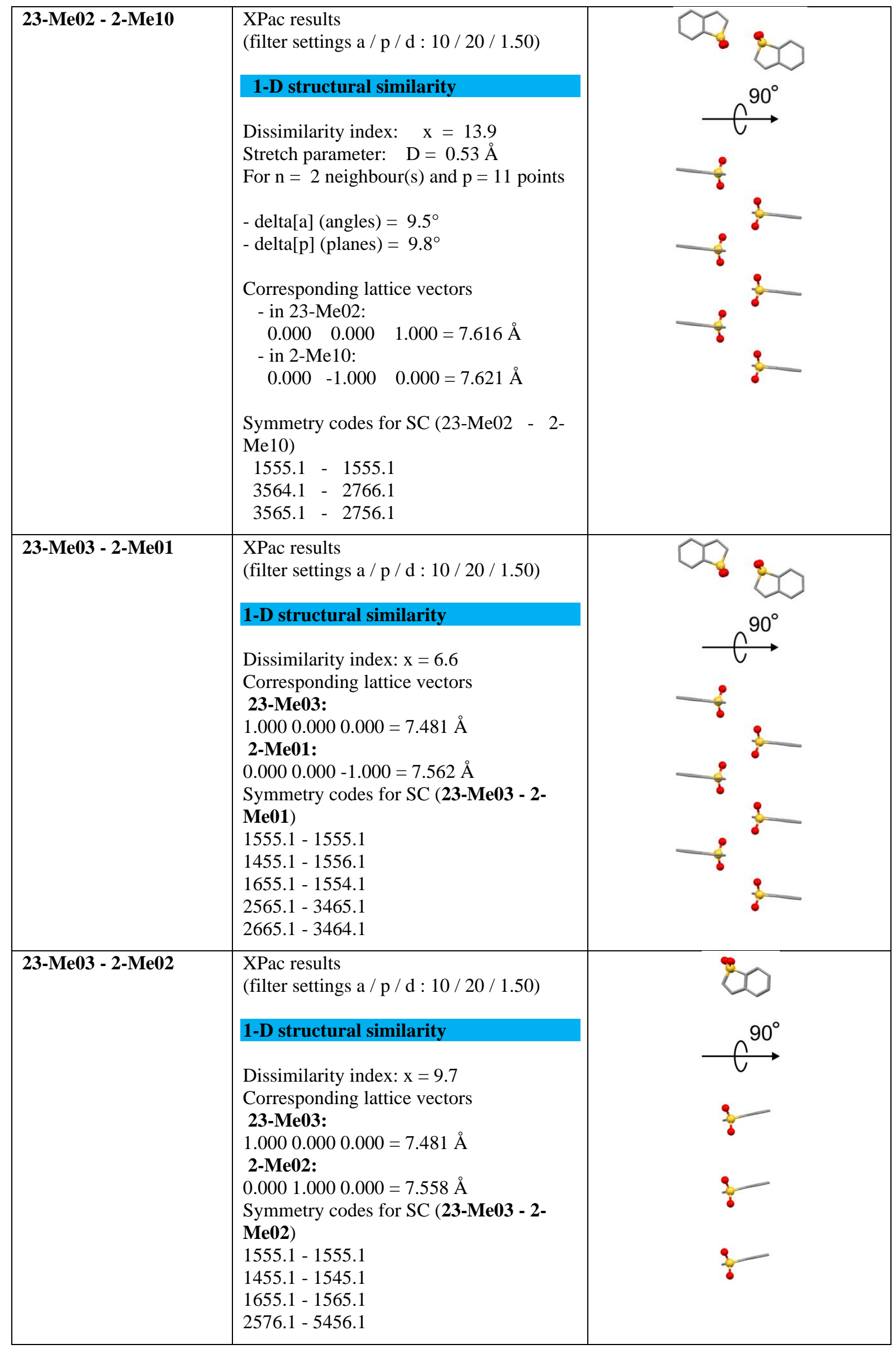




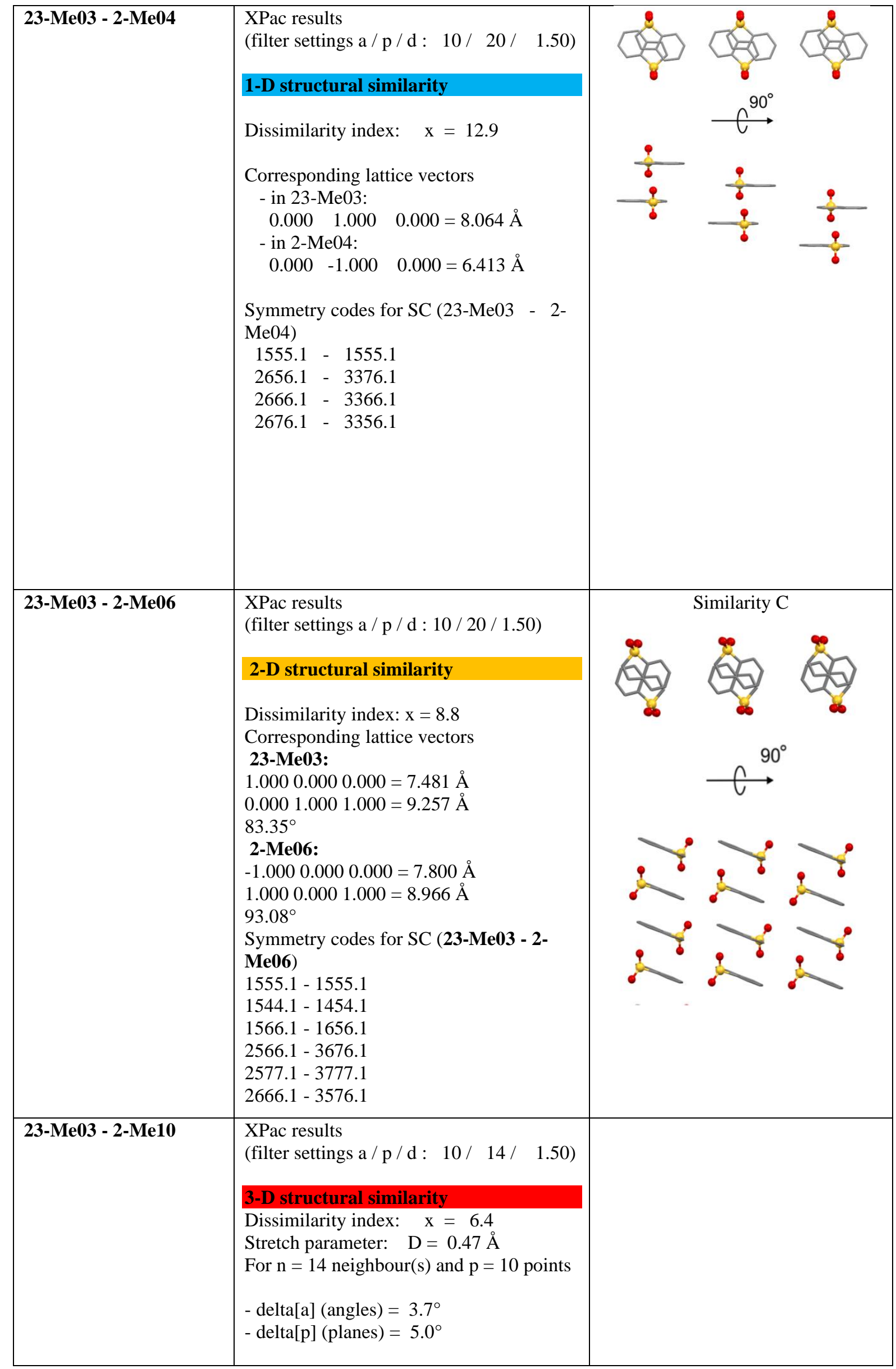




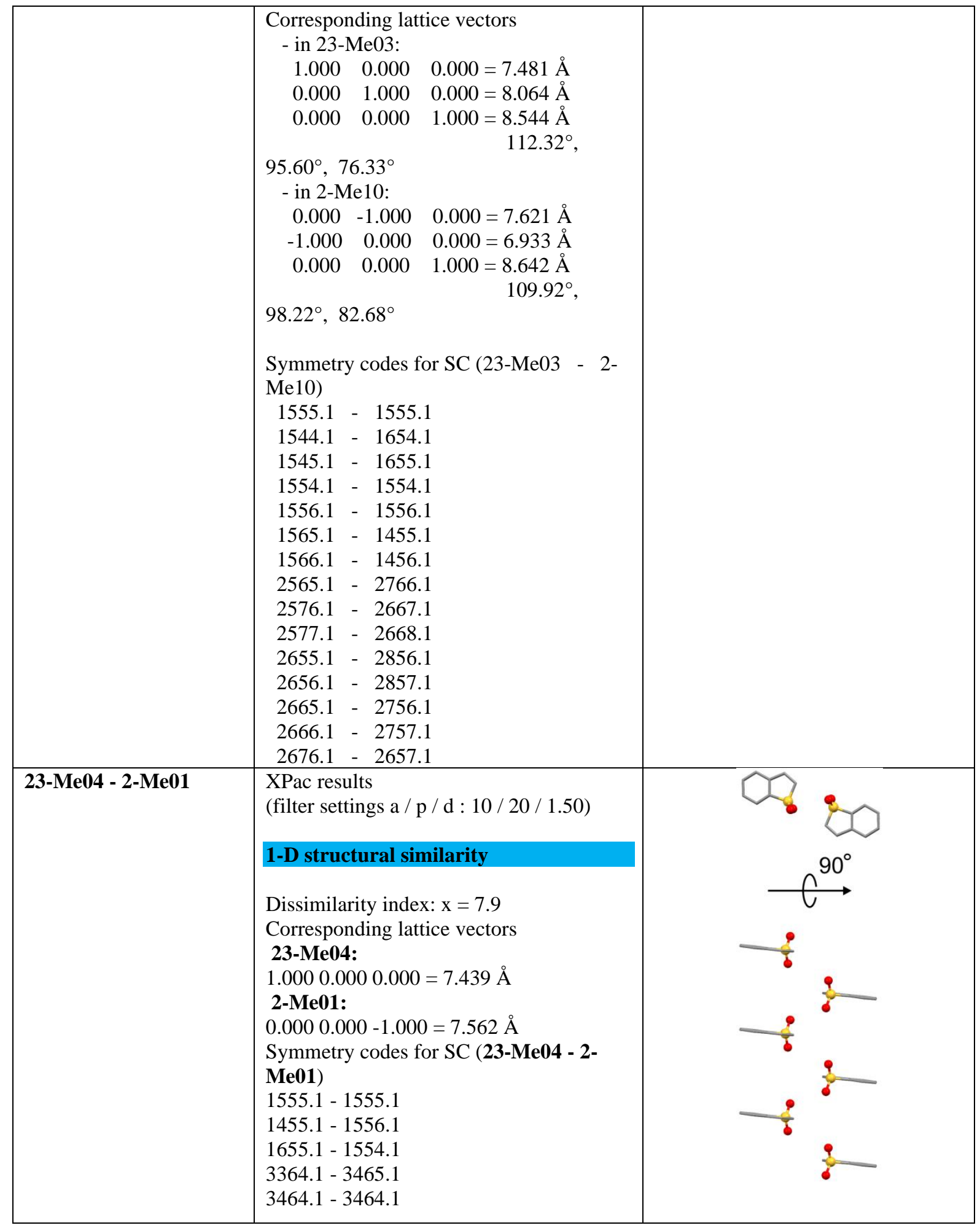




\begin{tabular}{|c|c|c|}
\hline 23-Me04 - 2-Me02 & 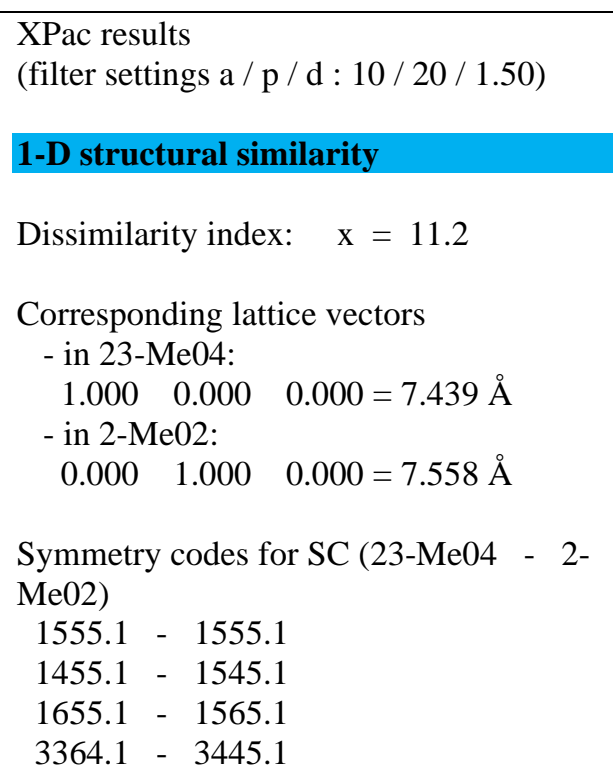 & $\underset{\frac{2}{2}}{\stackrel{2}{2}}$ \\
\hline 23-Me04 - 2-Me05 & 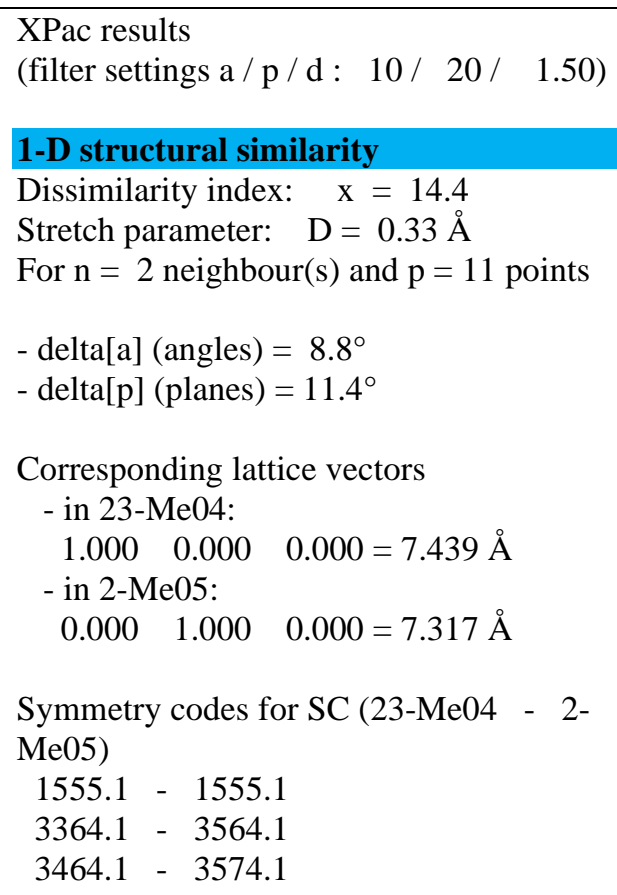 & 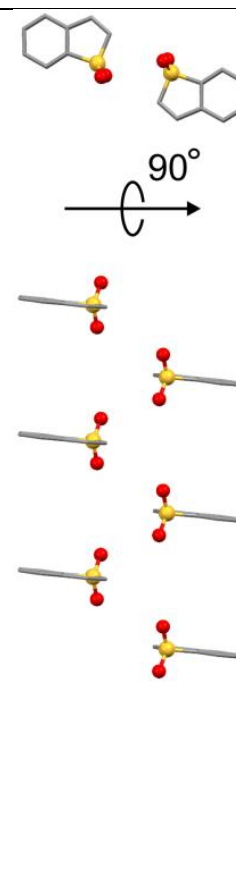 \\
\hline
\end{tabular}




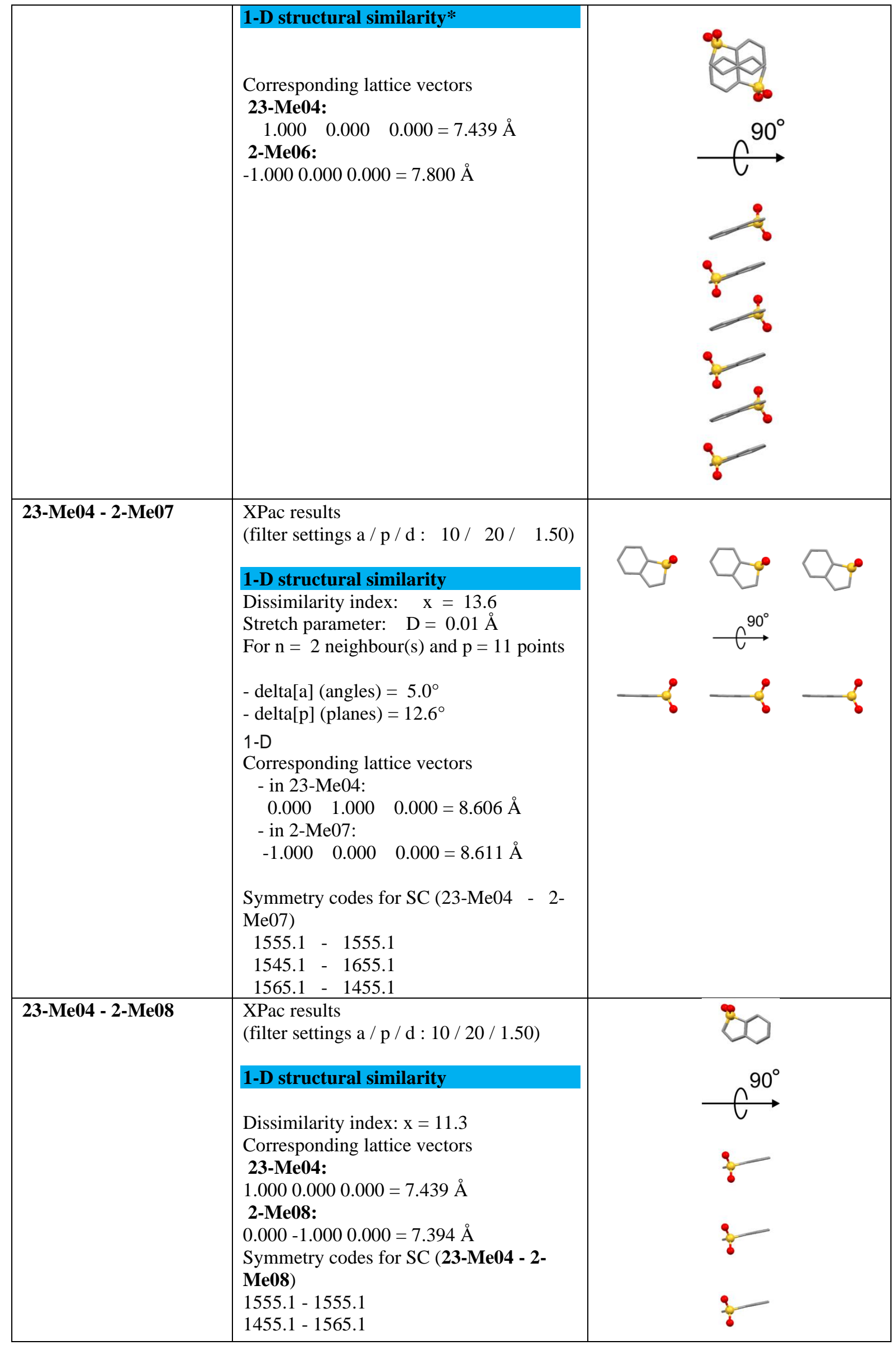




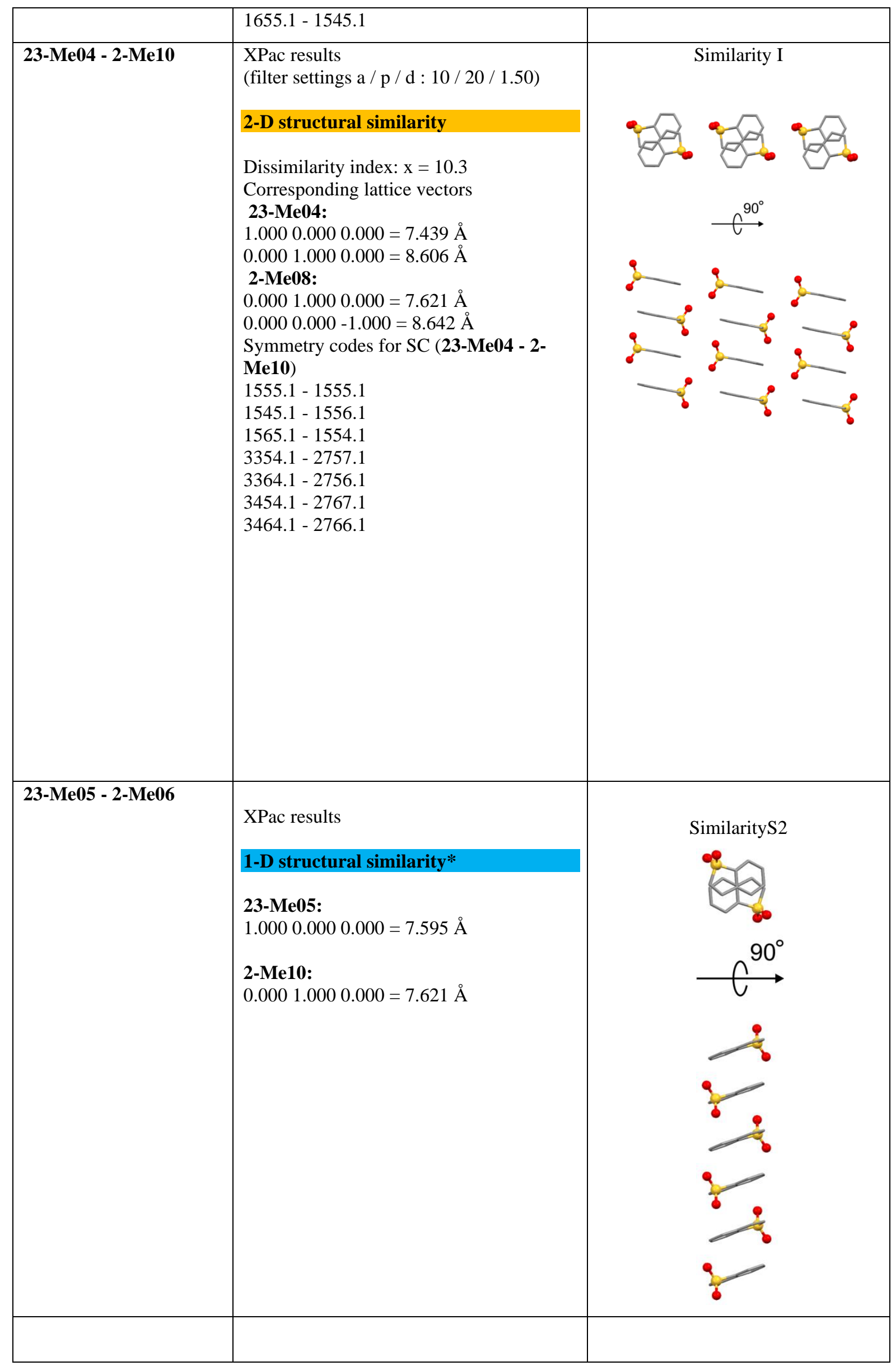




\begin{tabular}{|c|c|c|}
\hline 23-Me05 - 2-Me10 & $\begin{array}{l}\text { XPac results } \\
\text { 1-D structural similarity* } \\
\text { Corresponding lattice vectors } \\
\text { 23-Me05: } \\
\text { 1.000 0.000 } 0.000=7.595 \AA \\
\text { 2-Me10: } \\
0.0001 .0000 .000=7.621 \AA\end{array}$ & Similarity S2 \\
\hline 23-Me06 - 2-Me01 & 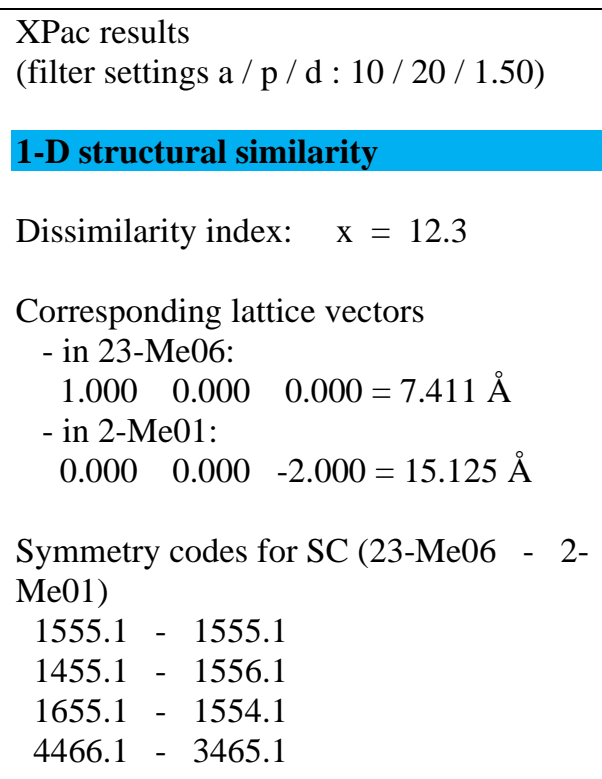 & $\frac{5}{6}$ \\
\hline 23-Me06 - 2-Me02 & $\begin{array}{l}\text { XPac results } \\
\text { (filter settings a / p / d : } 10 / 20 \text { / 1.50) } \\
\text { 1-D structural similarity } \\
\text { Dissimilarity index: } x=4.0 \\
\text { Corresponding lattice vectors } \\
\text { 23-Me06: } \\
\text { 1.000 0.000 } 0.000=7.411 \AA \\
\text { 2-Me02: } \\
0.0001 .0000 .000=7.558 \AA \\
\text { Symmetry codes for SC }(\mathbf{2 3 - M e 0 6 - 2 -} \\
\text { Me02) } \\
\text { 1555.1 - } 1555.1 \\
\text { 1455.1 - } 1545.1 \\
1655.1-1565.1 \\
4466.1-3445.1\end{array}$ & $\frac{1}{6} \frac{1}{2}$ \\
\hline
\end{tabular}




\begin{tabular}{|c|c|c|}
\hline & $4566.1-3455.1$ & \\
\hline 23-Me06 - 2-Me05 & 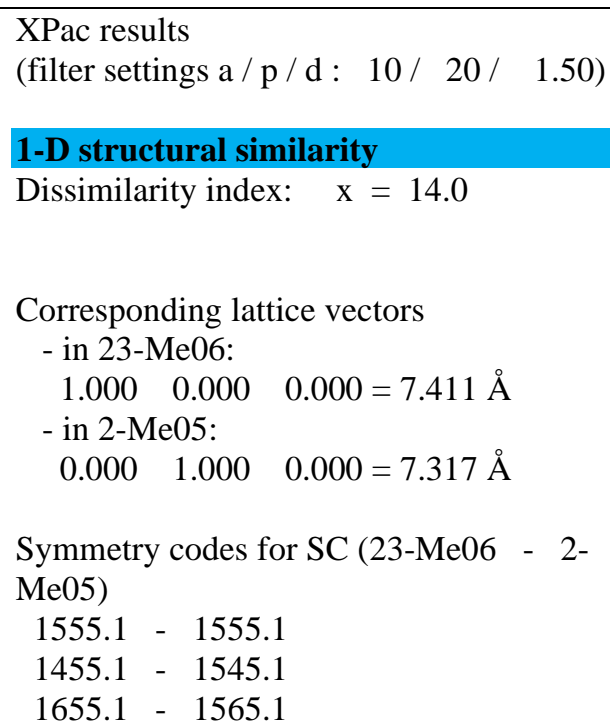 & \\
\hline 23-Me06 - 2-Me08 & 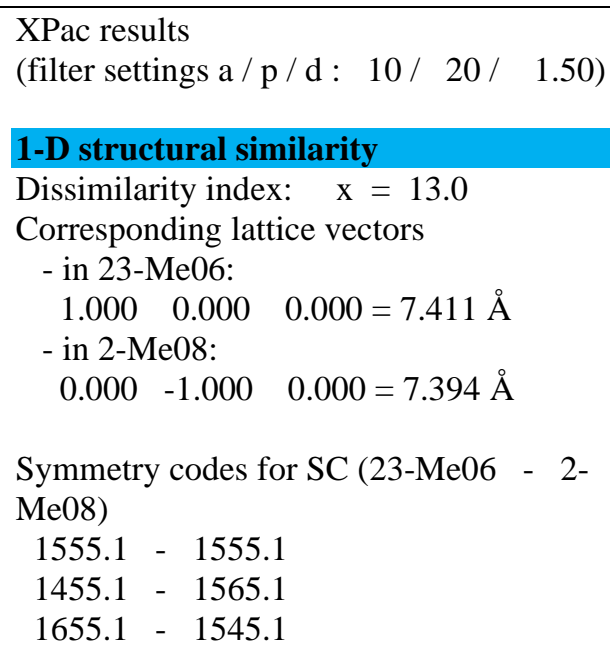 & 3 \\
\hline 23-Me07 - 2-Me01 & 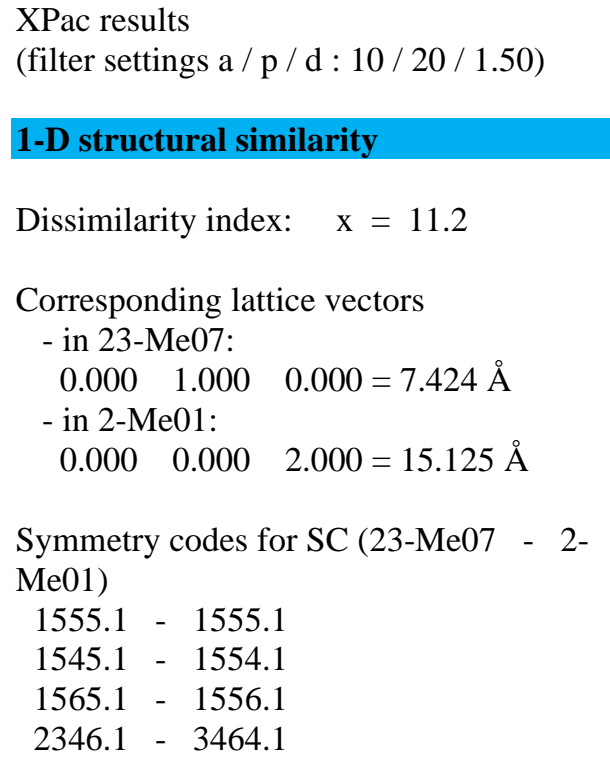 & $C_{\frac{2}{6}}^{90}$ \\
\hline
\end{tabular}




\begin{tabular}{|c|c|c|}
\hline 23-Me07 - 2-Me02 & 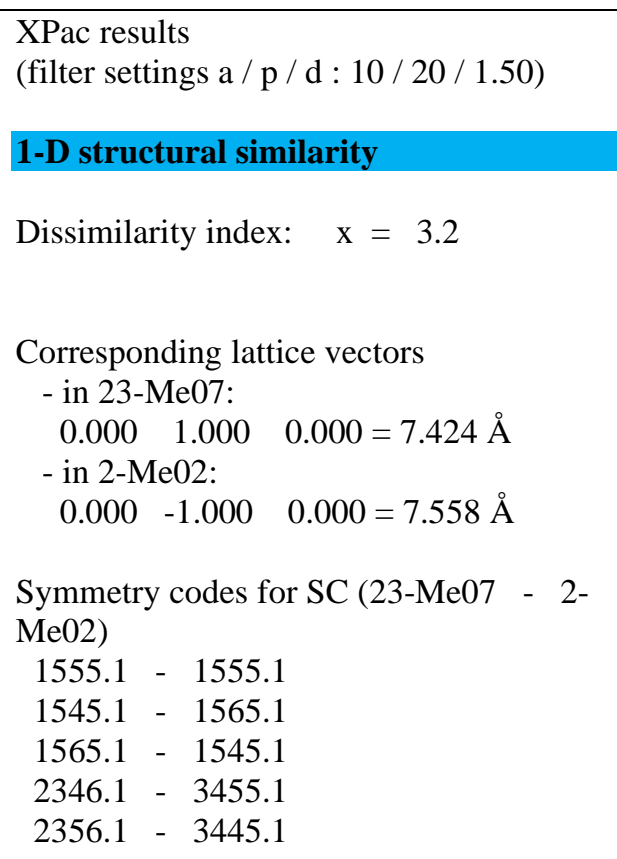 & $\frac{2}{8} \frac{2}{2}$ \\
\hline 23-Me07 - 2-Me05 & 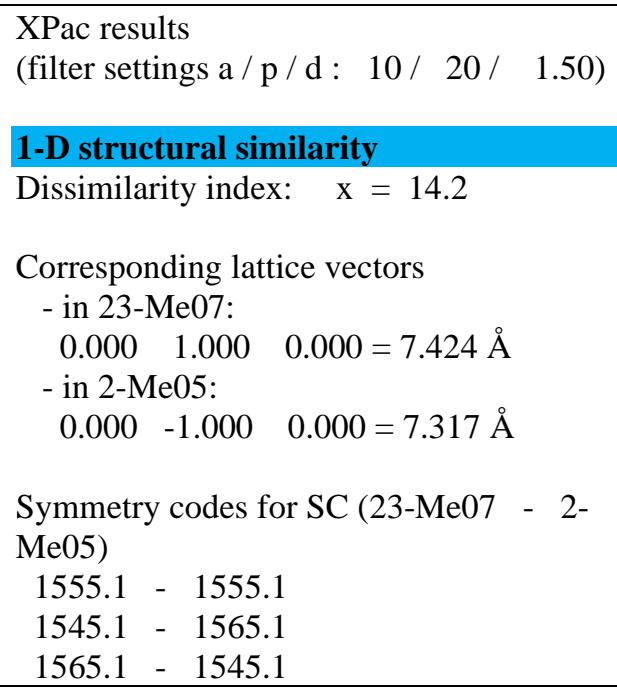 & 2 \\
\hline 23-Me07 - 2-Me08 & 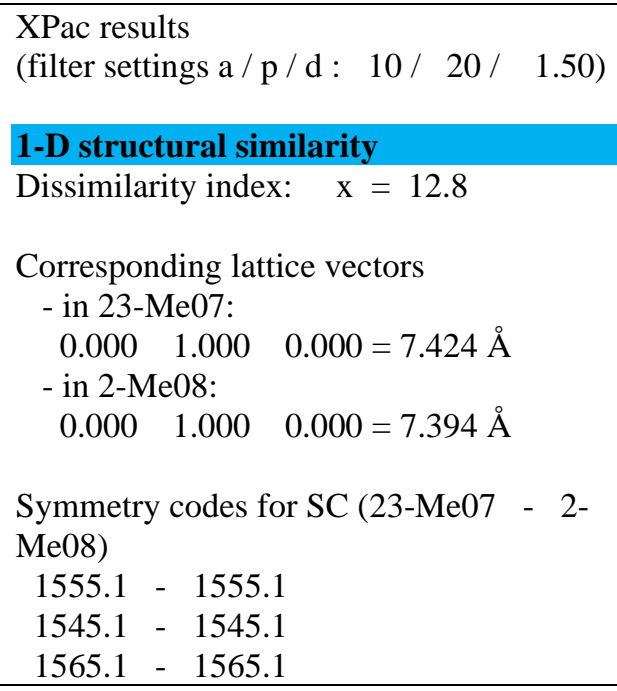 & $\frac{9}{6}$ \\
\hline 23-Me07 - 2-Me10 & $\begin{array}{l}\text { XPac results } \\
\text { (filter settings a / p / : } 15 / 20 / 4.00 \text { ) }\end{array}$ & Similarity I \\
\hline
\end{tabular}




\begin{tabular}{|c|c|c|}
\hline & 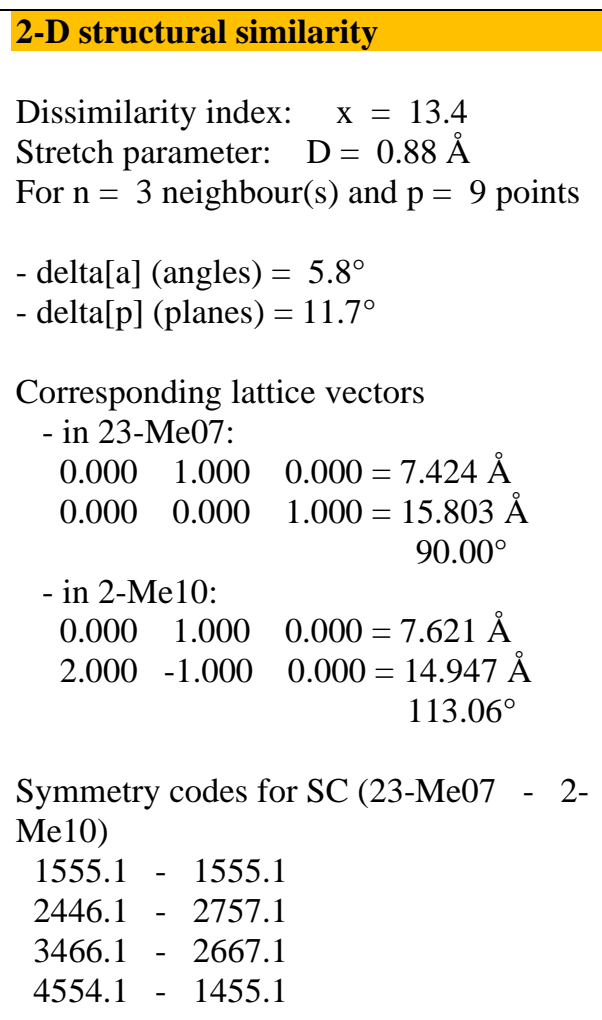 & $\underset{\infty}{\stackrel{9}{9}{ }_{\infty}^{\circ}}$ \\
\hline 23-Me08 - 2-Me01 & 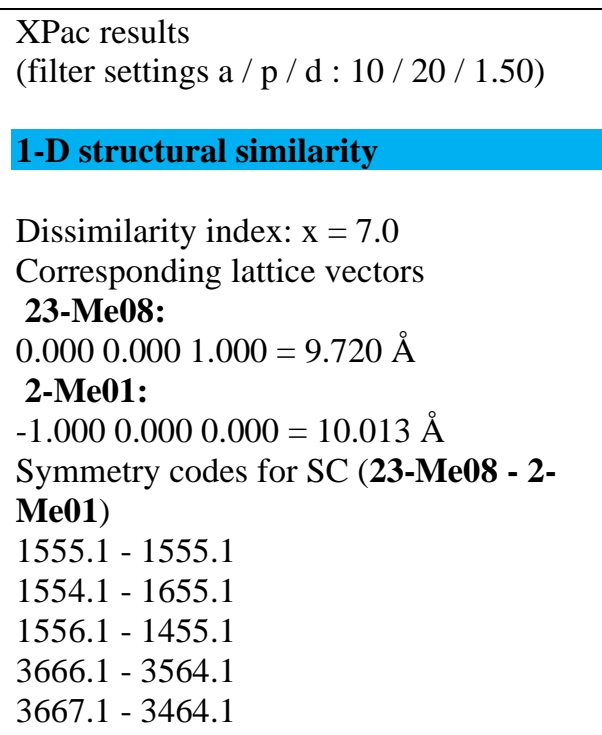 & 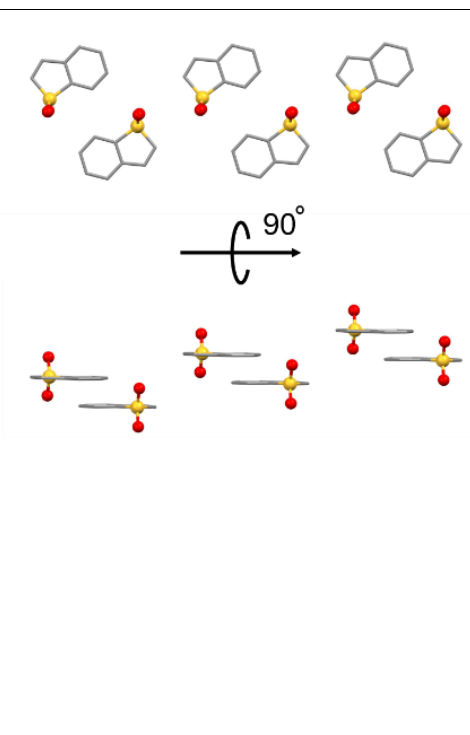 \\
\hline 23-Me08 - 2-Me07 & $\begin{array}{l}\text { XPac results } \\
\text { (filter settings a / p / d : } 10 \text { / } 20 \text { / 1.50) } \\
\text { 1-D structural similarity } \\
\text { Dissimilarity index: } x=12.8 \\
\text { Corresponding lattice vectors } \\
\text { 23-Me08: } \\
\text { 1.000 0.000 } 1.000=8.547 \AA \\
\text { 2-Me07: } \\
\text {-1.000 0.000 } 0.000=8.611 \AA \\
\text { Symmetry codes for SC (23-Me08 - 2- } \\
\text { Me07) } \\
\text { 1555.1 - } 1555.1 \\
\text { 1454.1 - } 1655.1\end{array}$ & $\frac{8}{6}$ \\
\hline
\end{tabular}




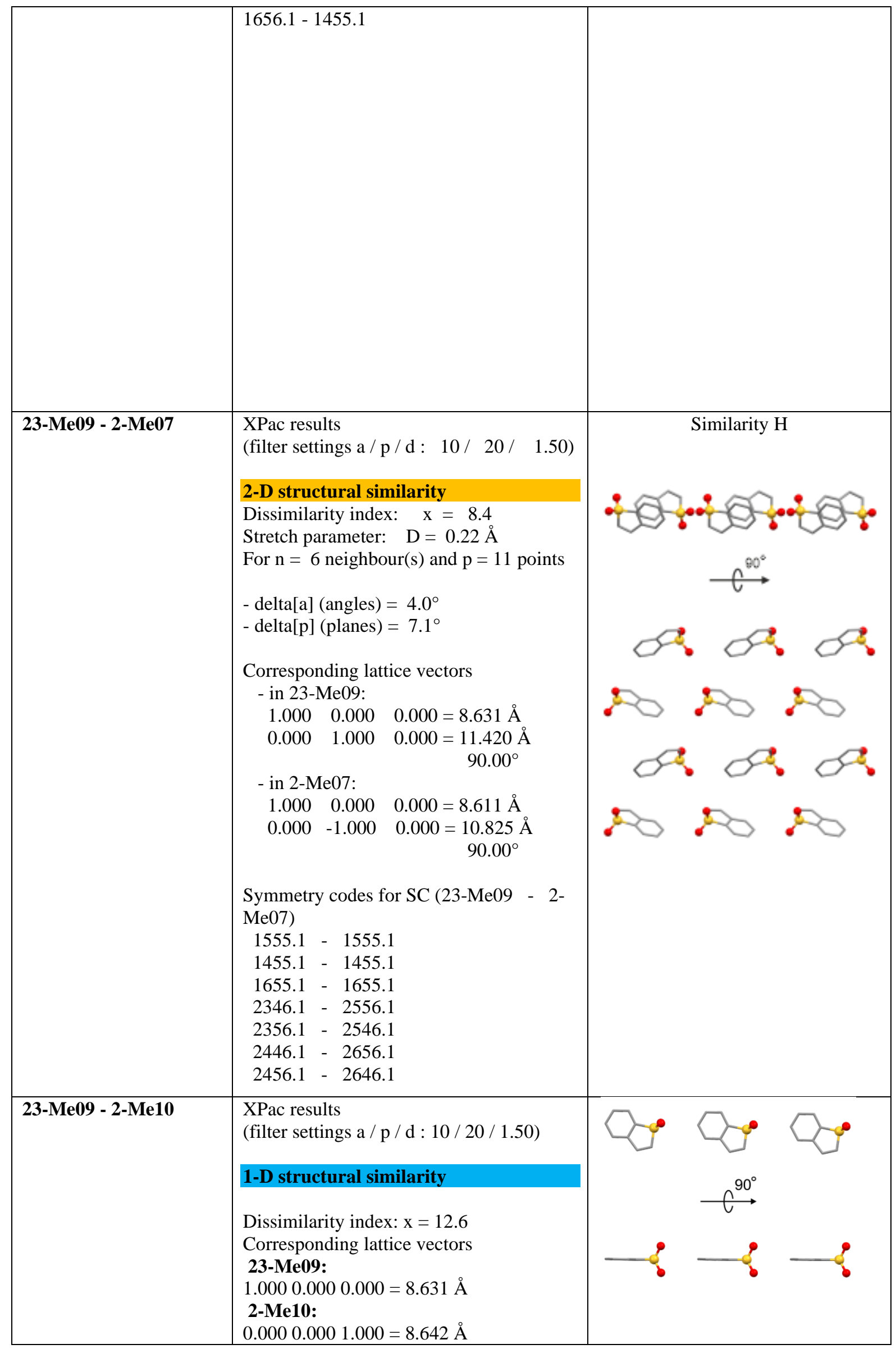




\begin{tabular}{|l|l|l|}
\hline & Symmetry codes for SC (23-Me09 - 2- & \\
Me10) & $1555.1-1555.1$ & \\
& $1455.1-1554.1$ & \\
$1655.1-1556.1$ & \\
\hline * Not identified within the filter parameters available in XPac & \\
\hline
\end{tabular}

Table S9. Comparison 23-Me_01-10 / 3-Me_01-10.

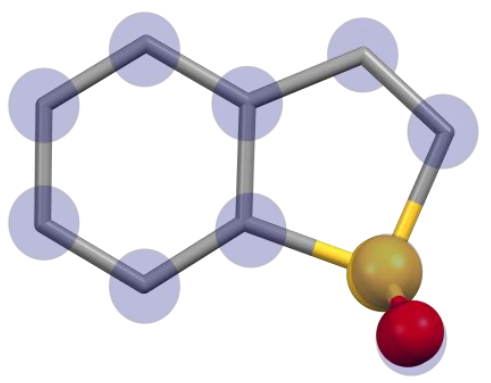

COSP

\begin{tabular}{|c|c|c|}
\hline Comparison & Results & Description \\
\hline 23-Me01 - 3-Me01 & 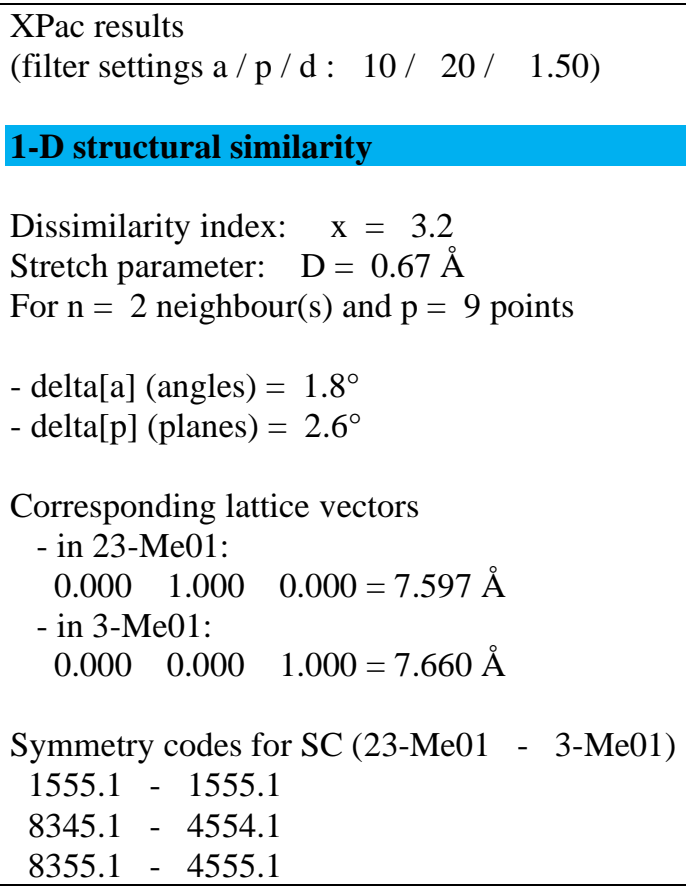 & Similarity S1 \\
\hline 23-Me01 - 3-Me03 & $\begin{array}{l}\text { XPac results } \\
\text { (filter settings a / p / d : } 12 / 18 \text { / 1.50) } \\
\text { 2-D structural similarity } \\
\text { Dissimilarity index: } x=1.9 \\
\text { Corresponding lattice vectors } \\
\text { 23-Me01: } \\
0.0001 .0000 .000=7.597 \AA \\
1.0000 .0000 .000=13.811 \AA \\
\end{array}$ & Similarity D \\
\hline
\end{tabular}




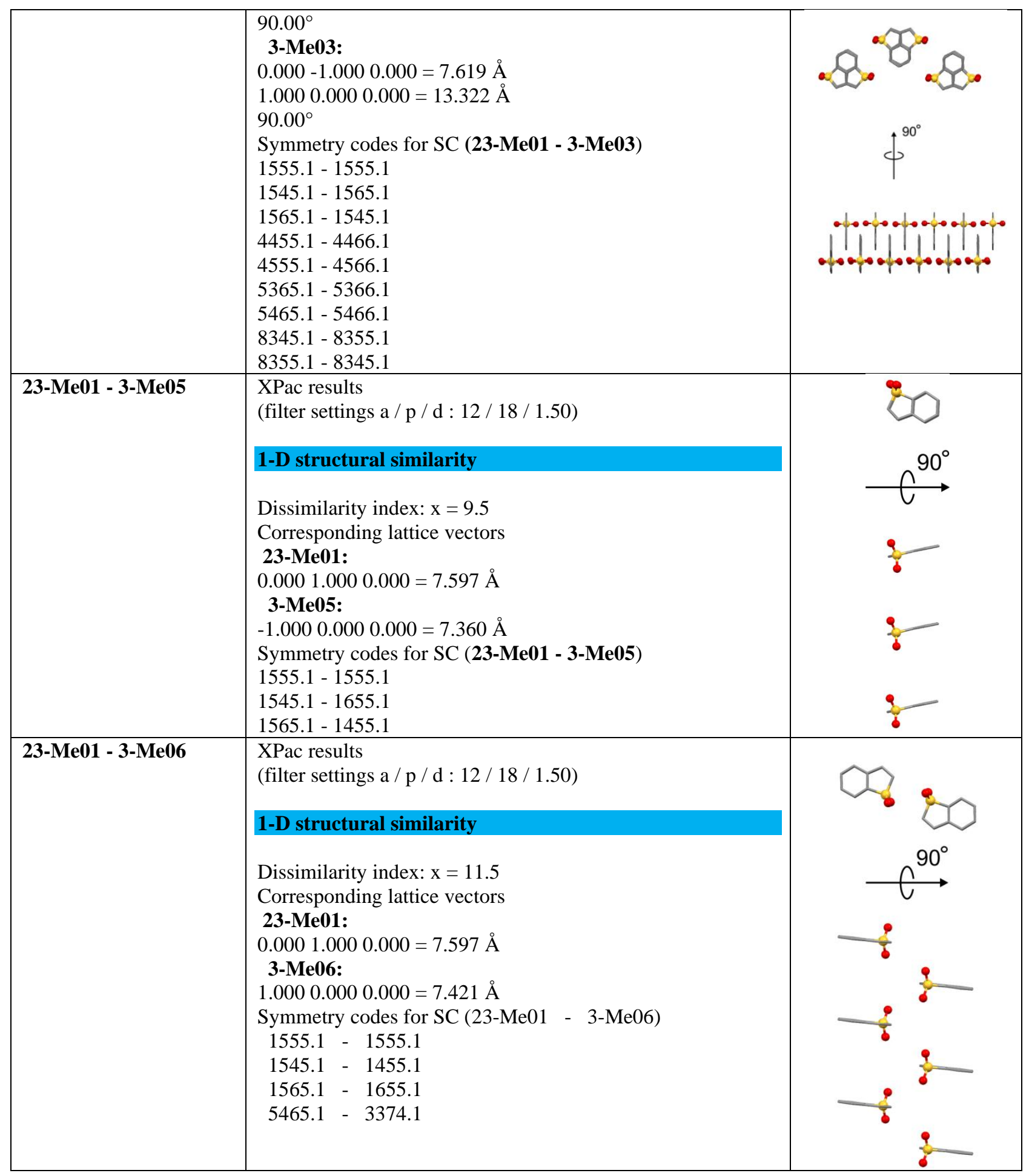




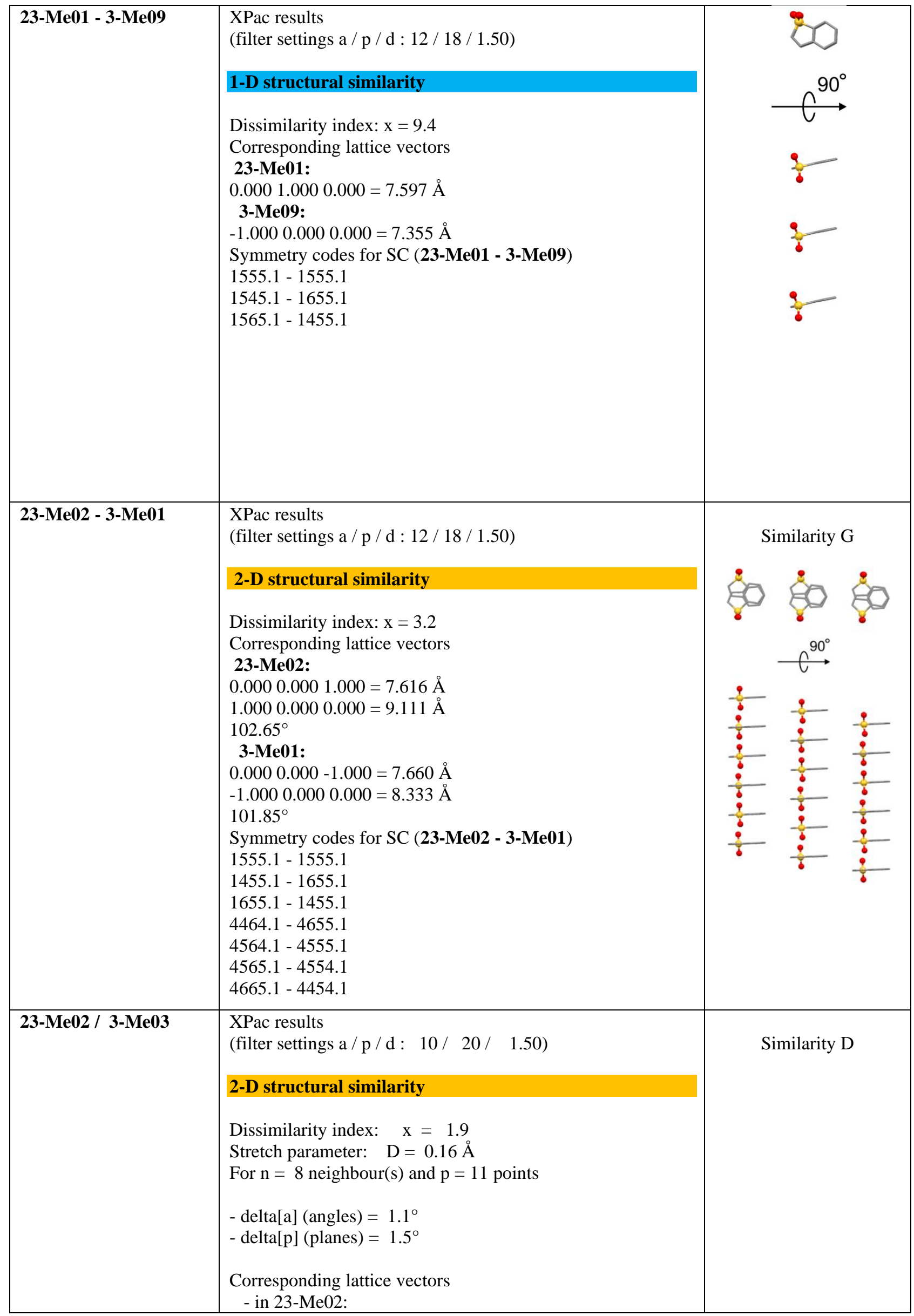




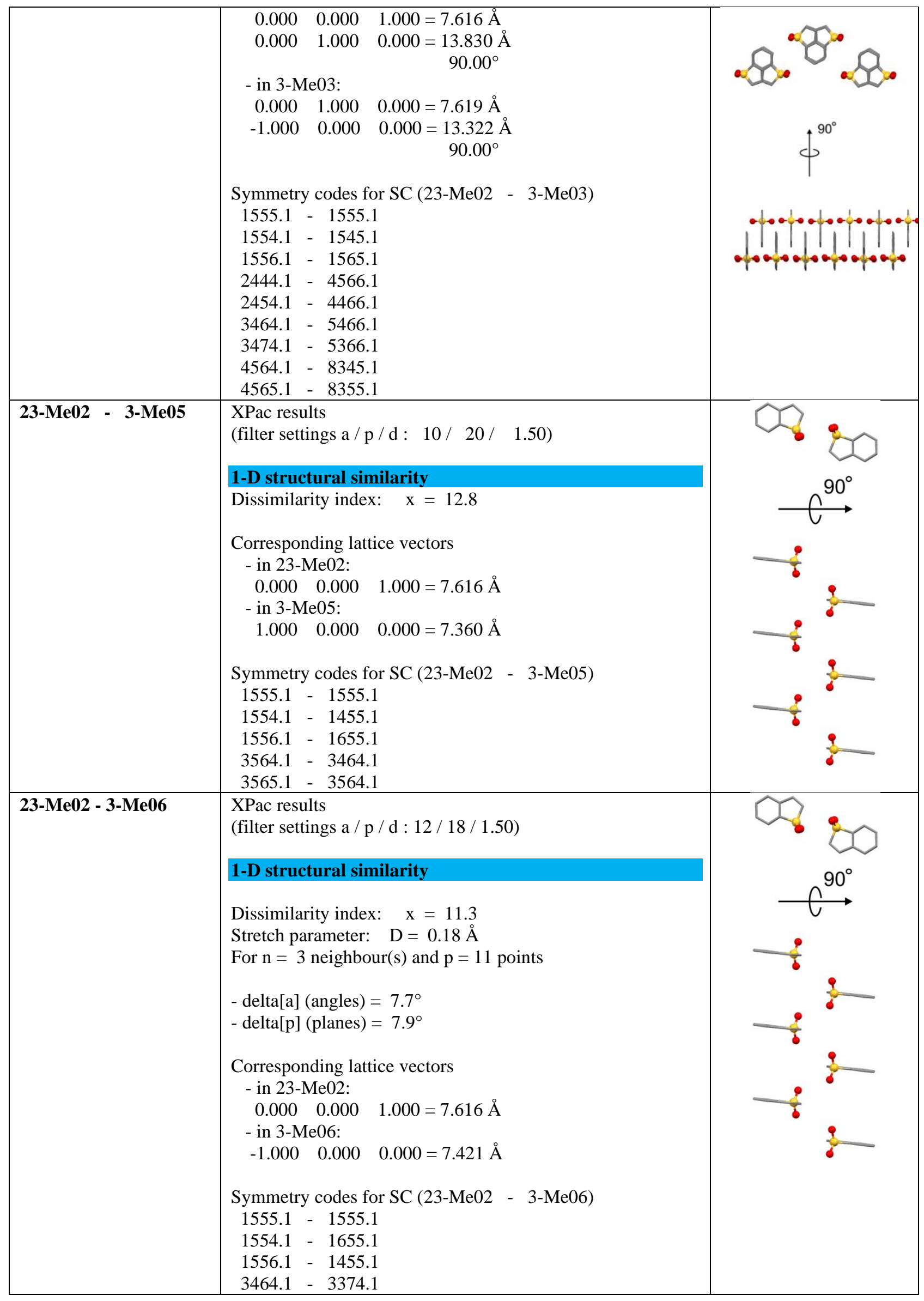




\begin{tabular}{|c|c|c|}
\hline 23-Me02 - 3-Me09 & 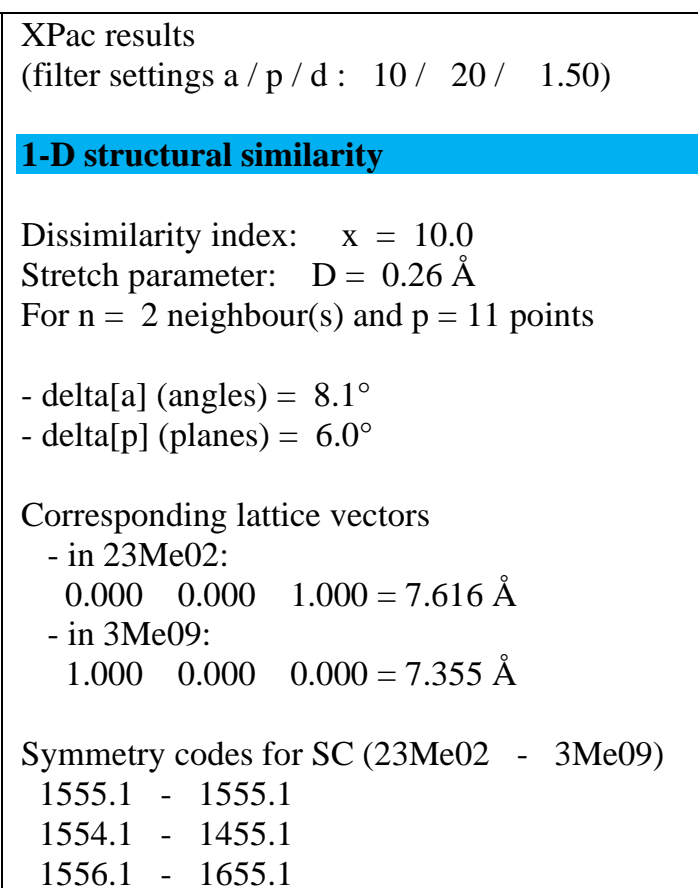 & 6 \\
\hline 23-Me03 - 3-Me03 & 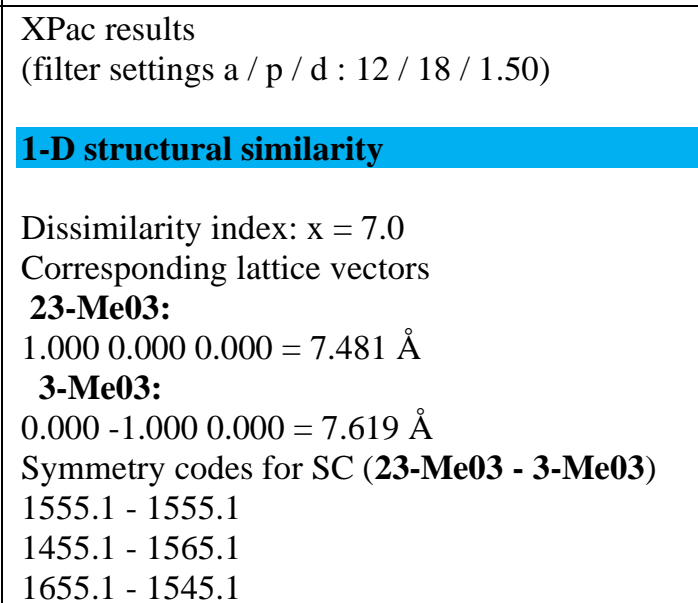 & \\
\hline 23-Me03 - 3-Me05 & 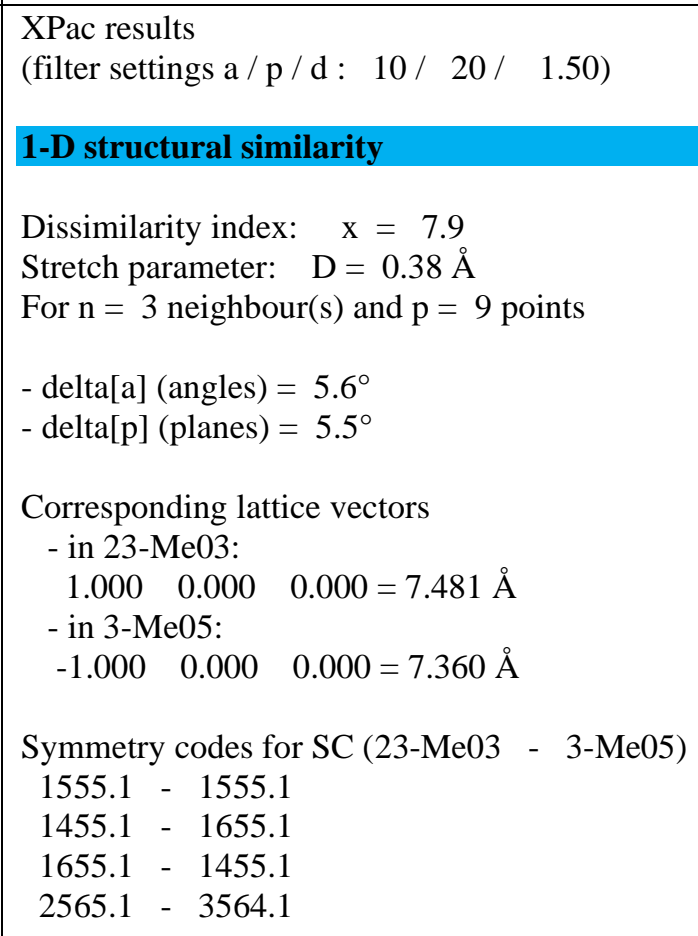 & Similarity S2 \\
\hline
\end{tabular}




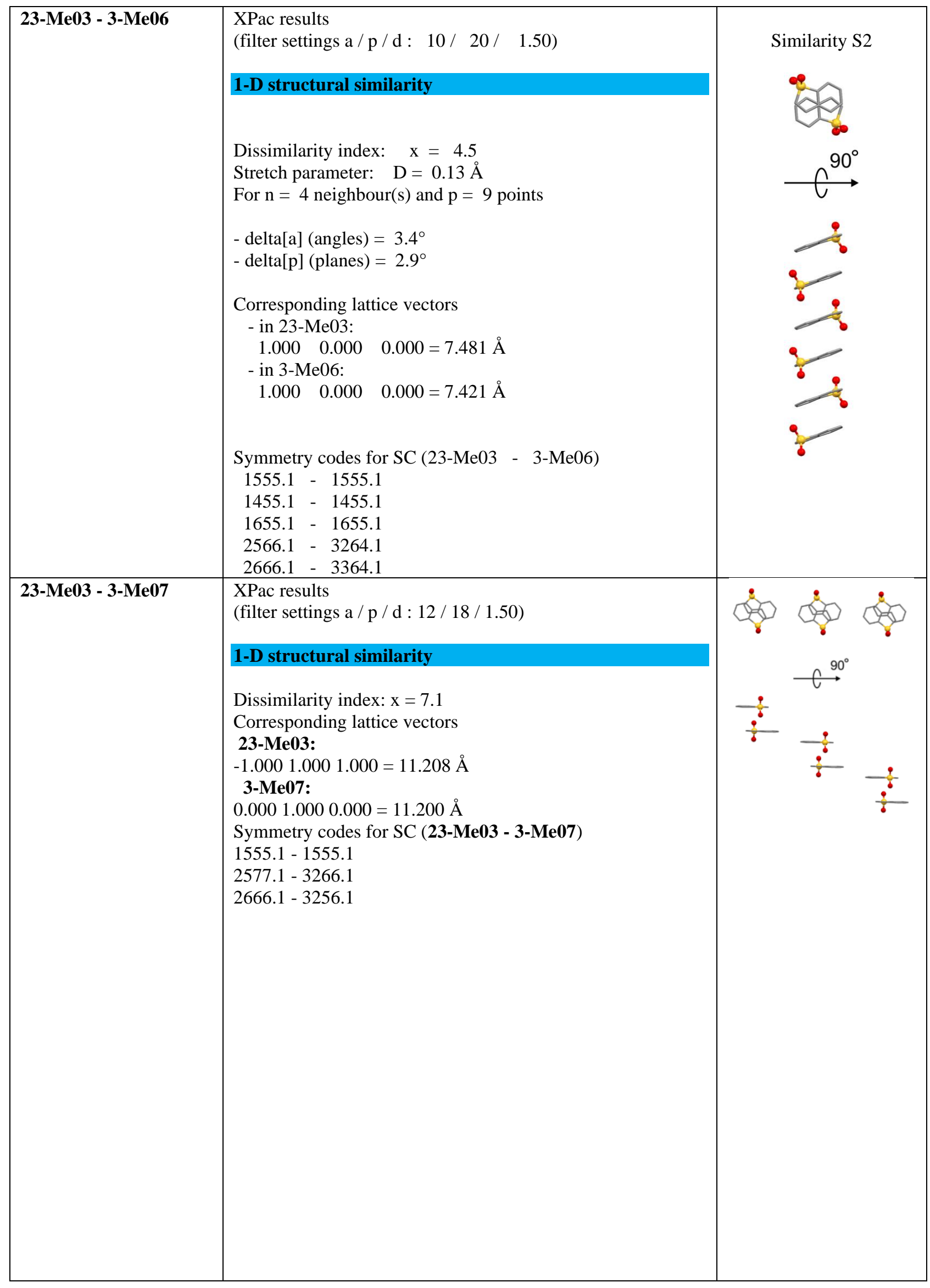




\begin{tabular}{|c|c|c|}
\hline 23-Me03 - 3-Me08 & 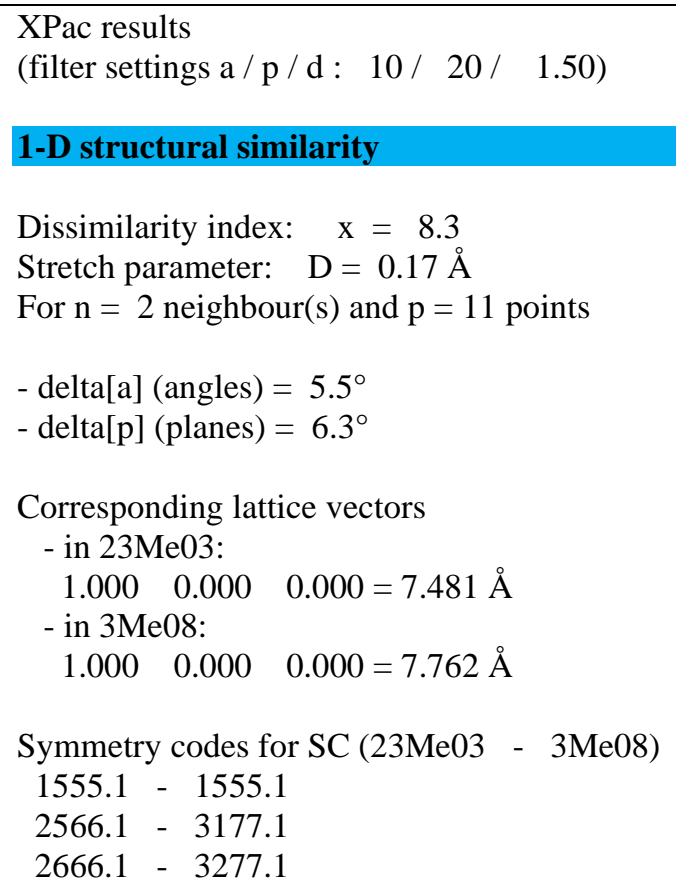 & Similarity S2 \\
\hline 23-Me03 - 3-Me09 & 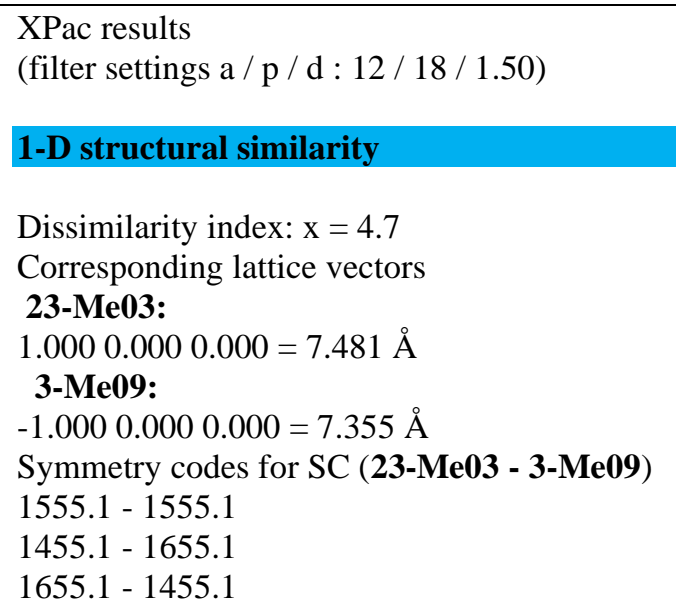 & \\
\hline 23-Me04 - 3-Me03 & 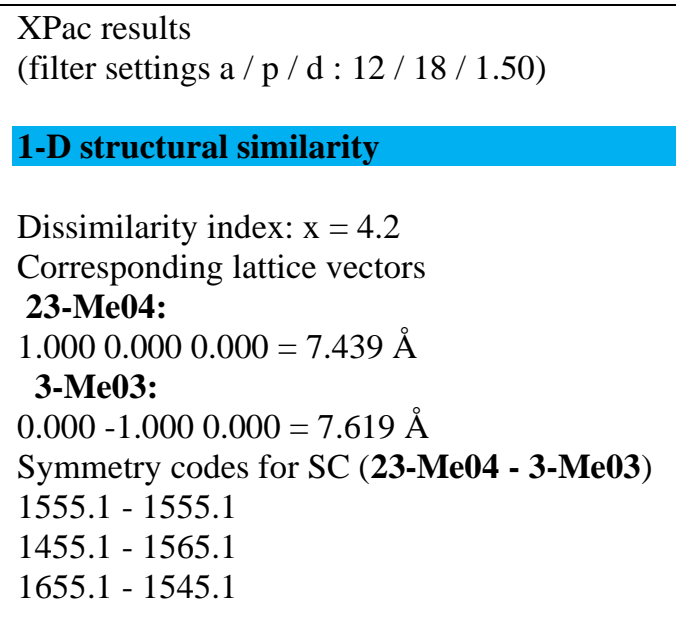 & $\frac{2}{6}$ \\
\hline
\end{tabular}




\begin{tabular}{|c|c|c|}
\hline 23-Me04 - 3-Me05 & 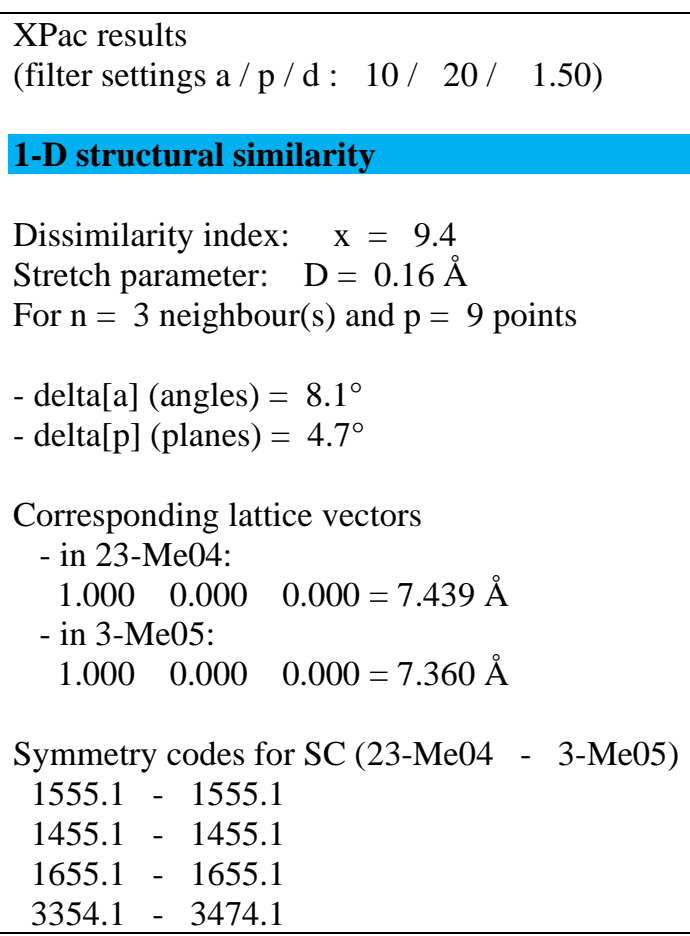 & Similarity S2 \\
\hline 23-Me04 - 3-Me06 & 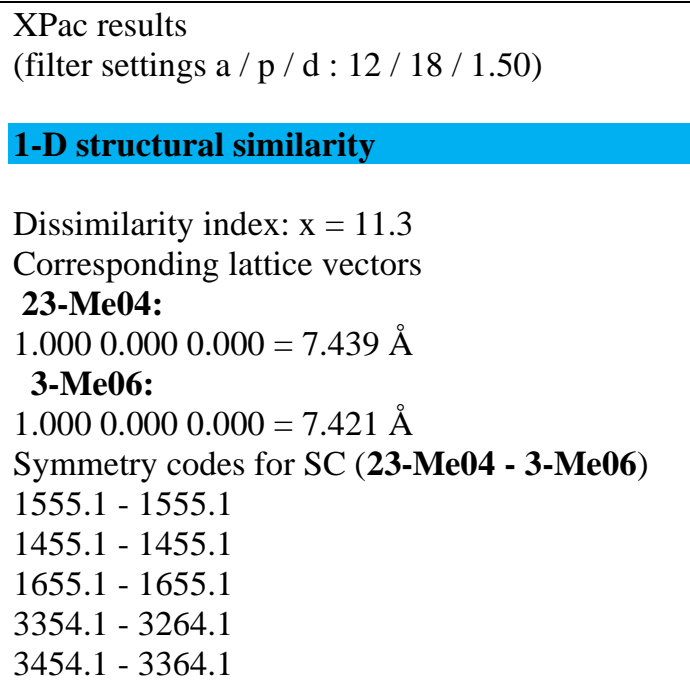 & Similarity S2 \\
\hline 23-Me04 - 3-Me08 & 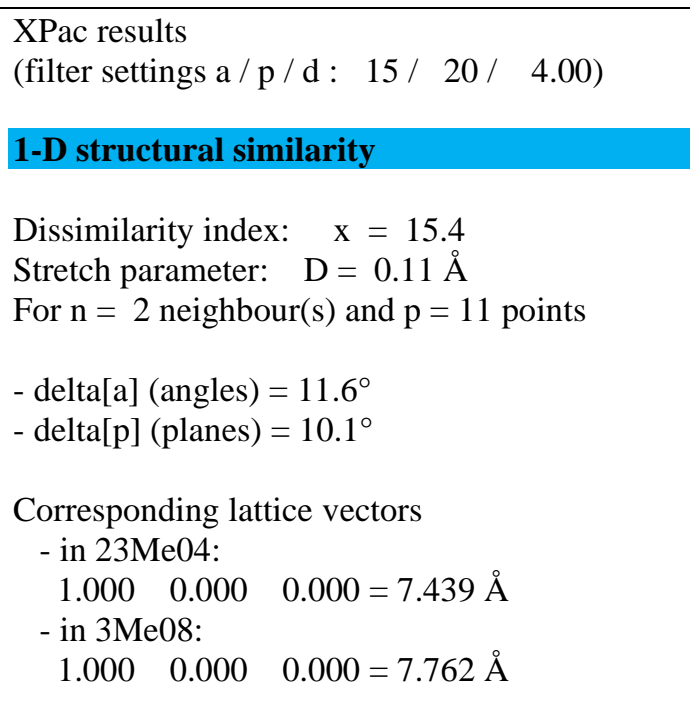 & Similarity S2 \\
\hline
\end{tabular}




\begin{tabular}{|c|c|c|}
\hline & $\begin{array}{ll}\text { Symmetry codes for SC }(23 \mathrm{Me} 04 & -3 \mathrm{Me} 08) \\
1555.1 & -1555.1 \\
3354.1 & -3177.1 \\
3454.1 & -3277.1\end{array}$ & \\
\hline 23-Me05 - 3-Me01 & $\begin{array}{l}\text { XPac results } \\
\text { (filter settings a / p / d : } 12 \text { / } 18 \text { / 1.50) } \\
\text { 1-D structural similarity } \\
\text { Dissimilarity index: } x=6.2 \\
\text { Corresponding lattice vectors } \\
\text { 23-Me05: } \\
0.0001 .0000 .000=8.711 \AA \\
\text { 3-Me01: } \\
1.0000 .0000 .000=8.333 \AA \\
\text { Symmetry codes for SC }(\mathbf{2 3 - M e 0 5 ~ - ~ 3 - M e 0 1 ) ~} \\
\text { 1555.1 - } 1555.1 \\
\text { 1545.1 - } 1455.1 \\
\text { 1565.1 - } 1655.1\end{array}$ & 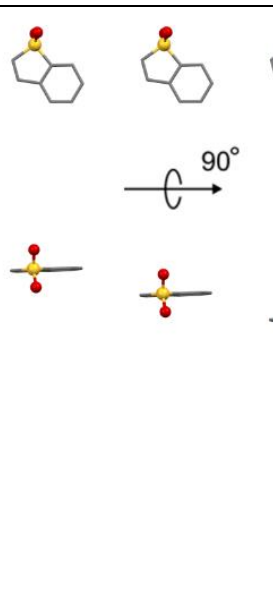 \\
\hline 23-Me05 - 3-Me05 & 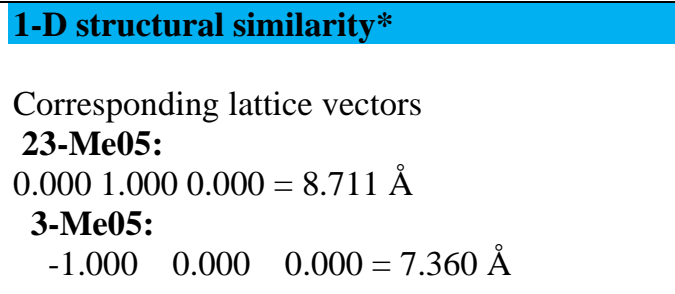 & Similarity S2 \\
\hline 23-Me05 - 3-Me06 & $\begin{array}{l}\text { 1-D structural similarity* } \\
\text { Corresponding lattice vectors } \\
\text { 23-Me05: } \\
\text { 0.000 1.000 } 0.000=8.711 \AA \\
\text { 3-Me06: } \\
\begin{array}{l}1.000 \quad 0.000 \quad 0.000=7.421 \AA\end{array}\end{array}$ & Similarity $\mathrm{S} 2$ \\
\hline
\end{tabular}




\begin{tabular}{|c|c|c|}
\hline 23-Me05 - 3-Me08 & 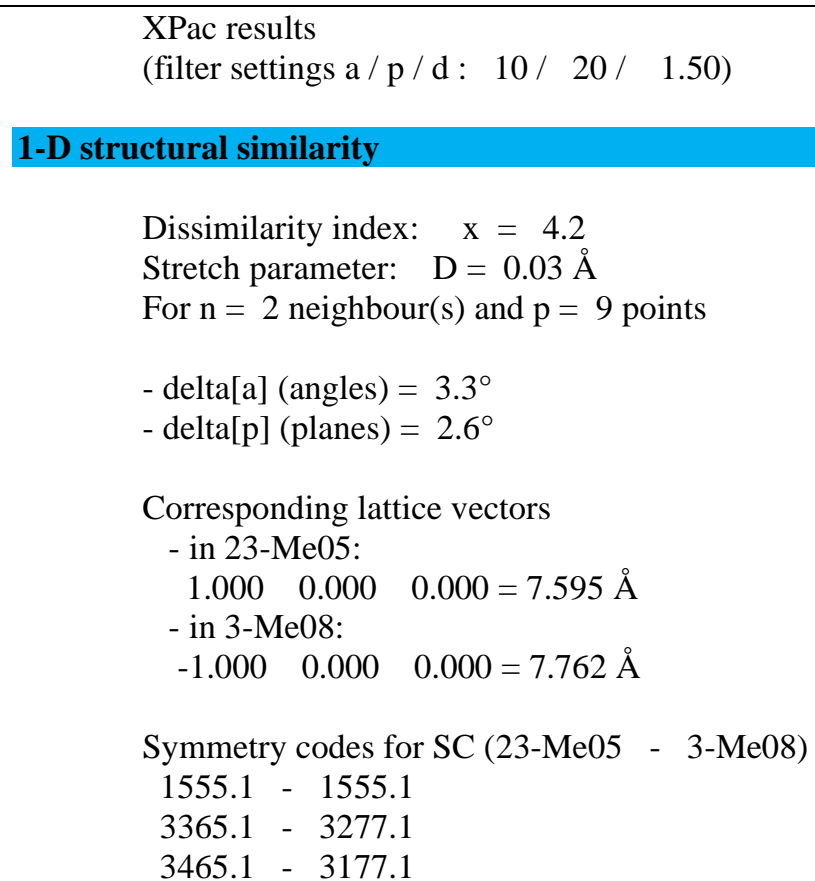 & Similarity S2 \\
\hline 23-Me06 - 3-Me03 & $\begin{array}{l}\text { XPac results } \\
\text { (filter settings a / p / d : } 12 \text { / } 18 \text { / 1.50) } \\
\text { 1-D structural similarity } \\
\text { Dissimilarity index: } \mathrm{x}=1.1 \\
\text { Corresponding lattice vectors } \\
\text { 23-Me06: } \\
\text { 1.000 0.000 } 0.000=7.411 \AA \\
\text { 3-Me03: } \\
0.000-1.0000 .000=7.619 \AA \\
\text { Symmetry codes for SC }(\mathbf{2 3 - M e 0 6 - 3 - M e 0 3 )} \\
\text { 1555.1 - } 1555.1 \\
\text { 1455.1 - } 1565.1 \\
1655.1-1545.1\end{array}$ & $\frac{2}{6}$ \\
\hline 23-Me06 - 3-Me05 & 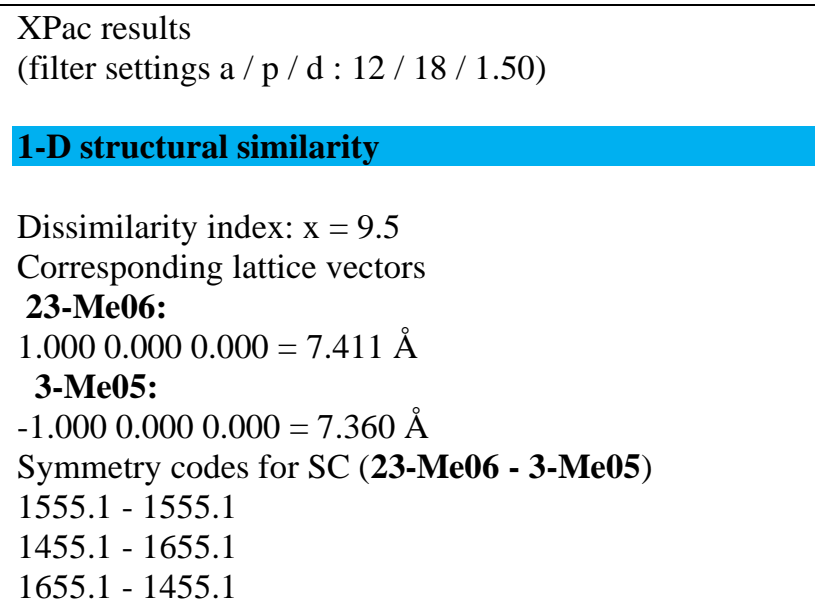 & $\frac{2}{2}$ \\
\hline
\end{tabular}




\begin{tabular}{|c|c|c|}
\hline 23-Me06 - 3-Me06 & 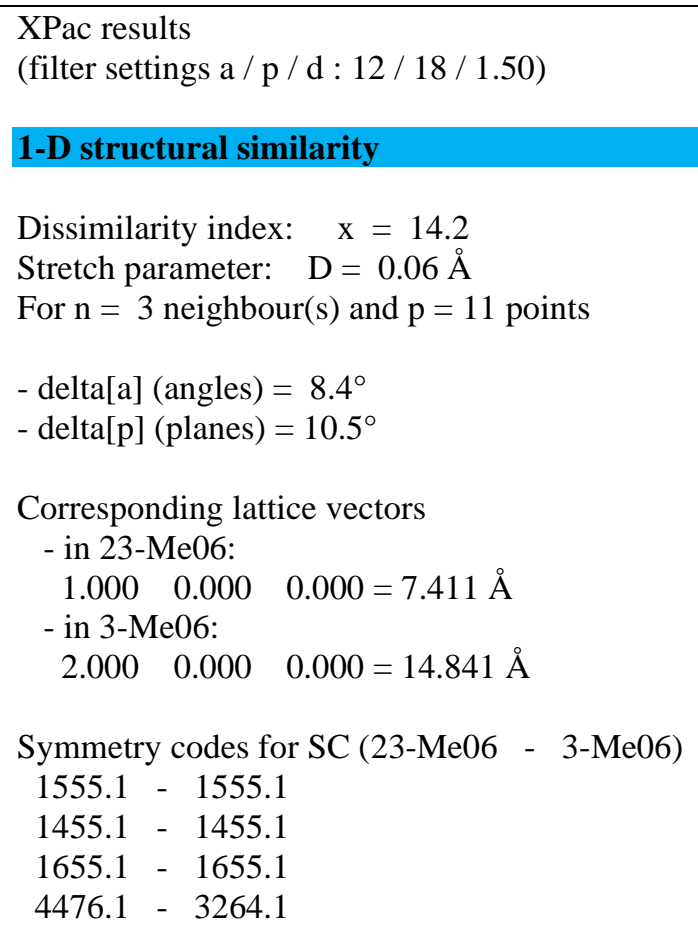 & Similarity S2 \\
\hline 23-Me06 - 3-Me09 & 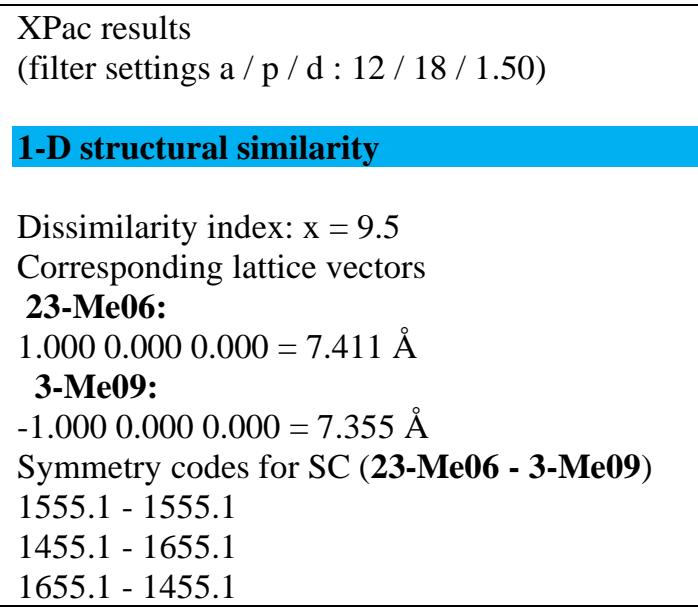 & 2 \\
\hline 23-Me07 - 3-Me03 & 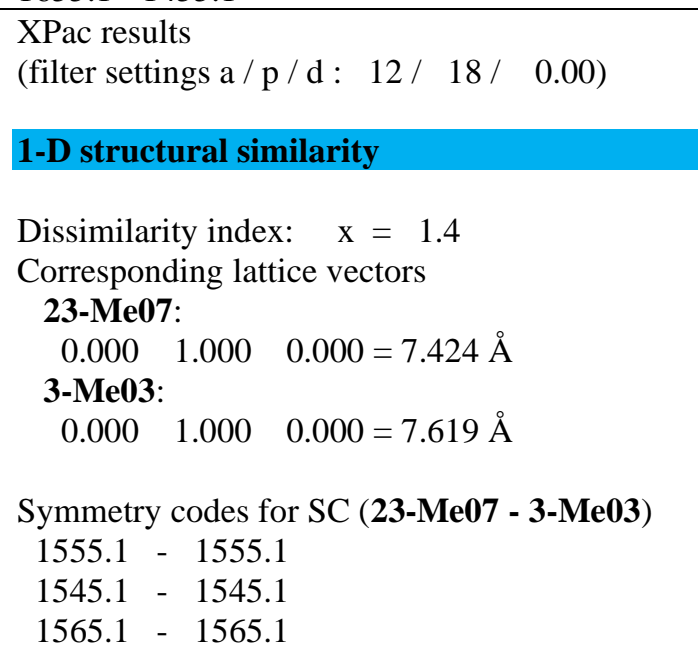 & 2 \\
\hline
\end{tabular}




\begin{tabular}{|c|c|c|}
\hline 23-Me07 - 3-Me05 & 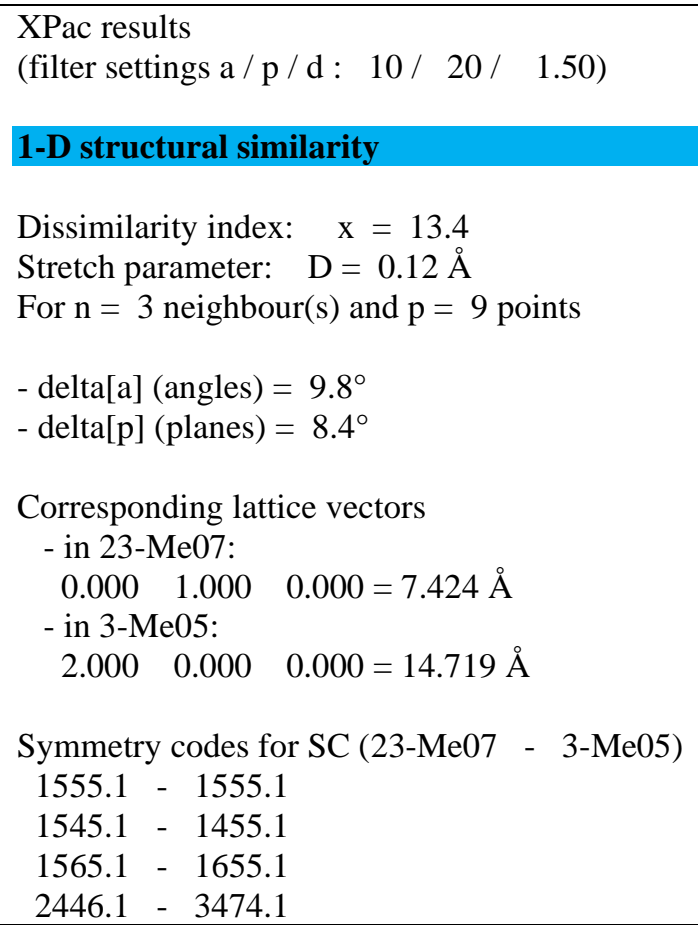 & Similarity S2 \\
\hline 23-Me07 - 3-Me06 & 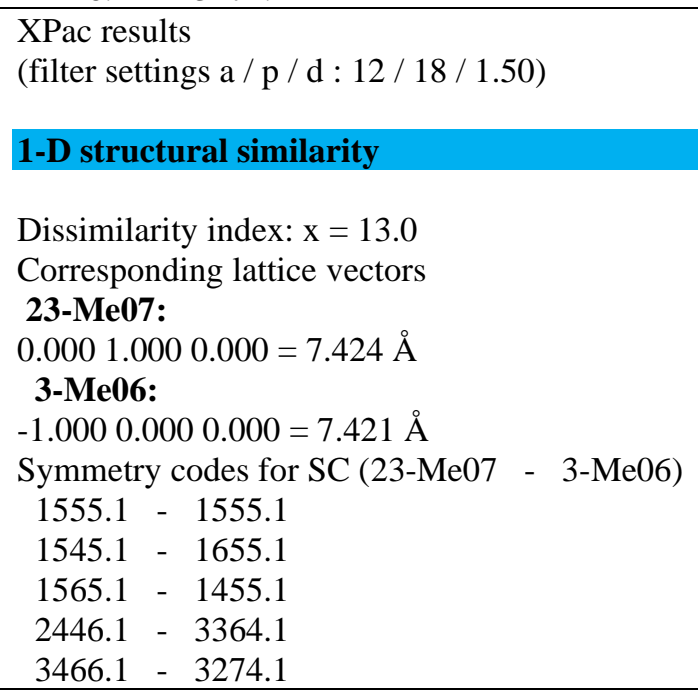 & $\begin{array}{l}\frac{8}{6} \\
\frac{8}{6}\end{array}$ \\
\hline 23-Me07 - 3-Me09 & 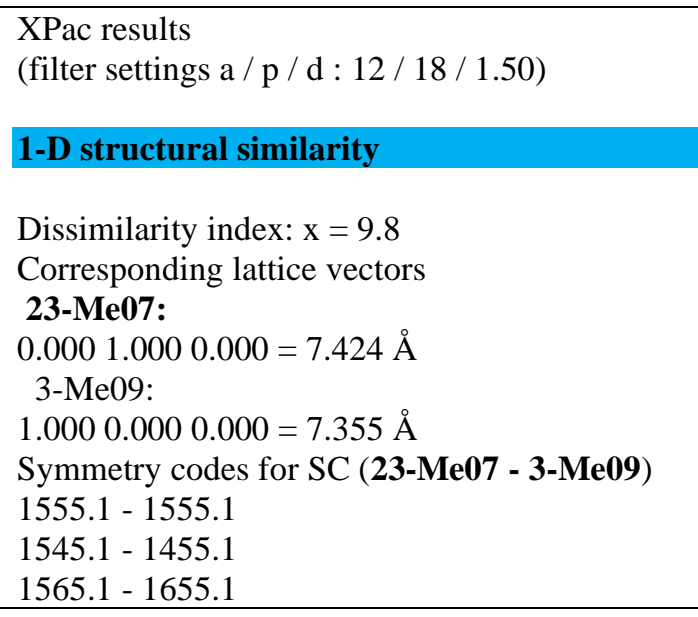 & 2 \\
\hline
\end{tabular}




\begin{tabular}{|c|c|c|}
\hline 23-Me08 - 3-Me01 & 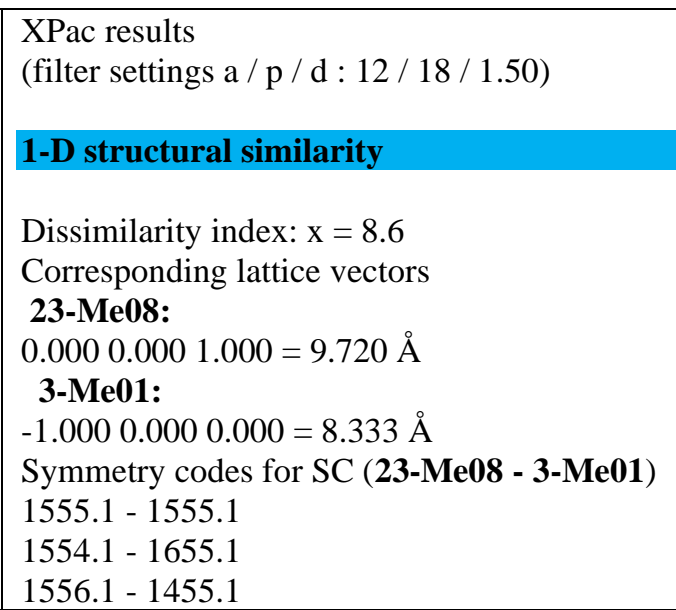 & $\stackrel{90^{\circ}}{\longrightarrow}$ \\
\hline 23-Me09 - 3-Me08 & 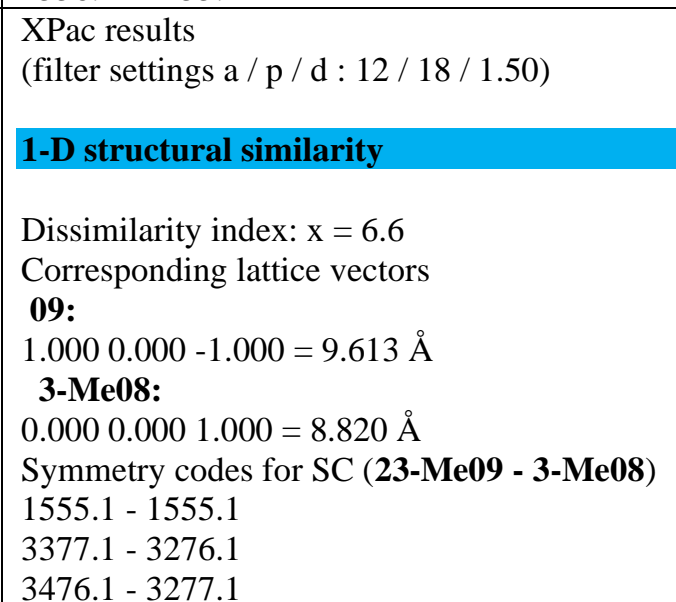 & $y=$ \\
\hline 23-Me10 - 3-Me01 & 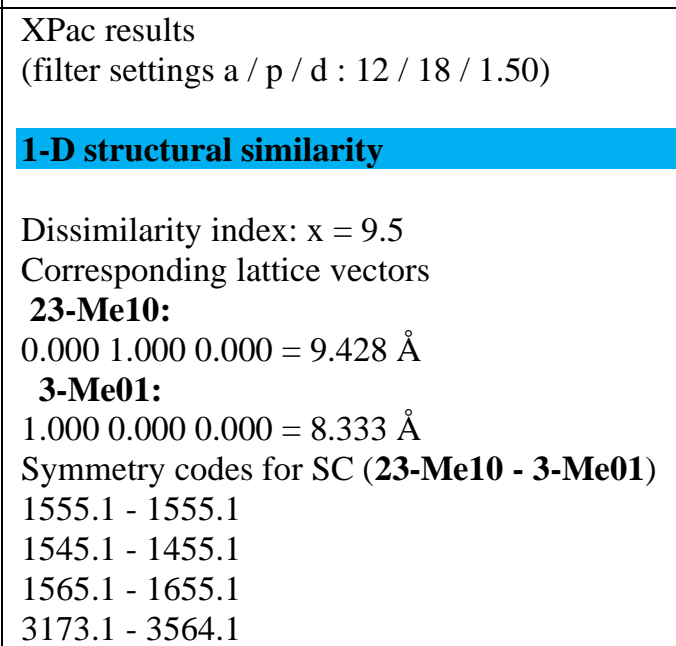 & $\stackrel{90^{\circ}}{\longrightarrow}$ \\
\hline
\end{tabular}




\section{S4. Crystal Packing Differences}

\section{S4.1. Similarity A}
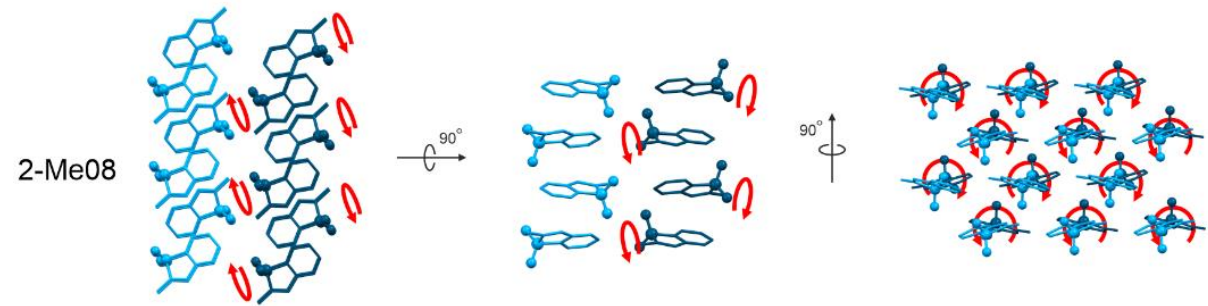

2-Me05
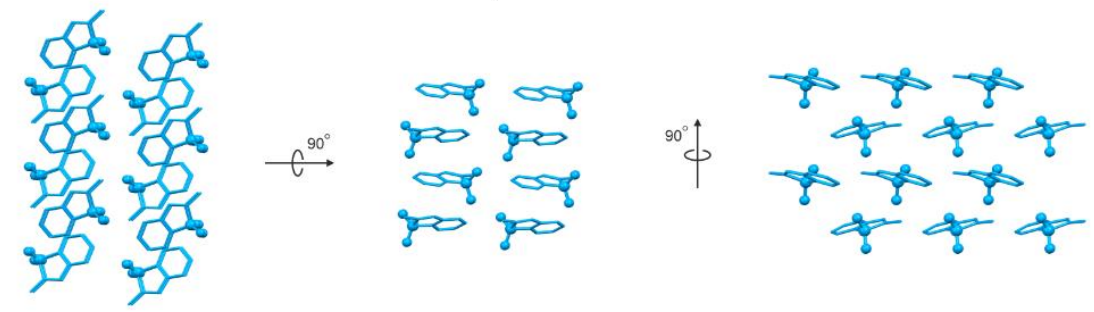

Figure S7. Crystal packing comparison for predictions showing the common similarity A, 2-Me08, 2-Me05. The packing instances are represented along three perpendicular directions, the molecules are colour coded according to the different orientation. Rotation of single molecules are indicated by red circles. These identify hypothetical transformations between the structures compared.

\section{S4.2. Similarity B}

2-Me09

B

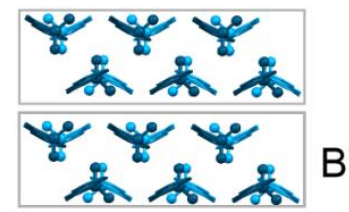

$B^{\prime}$<smiles>c1ccccc1</smiles>

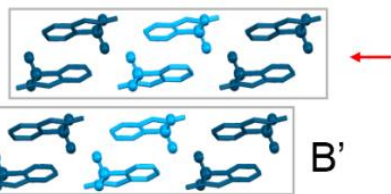

B

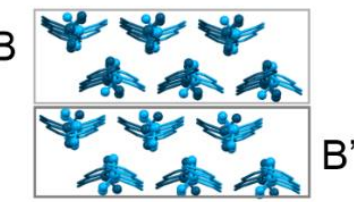<smiles>C1CC2C3CC1CC2C3</smiles>

B

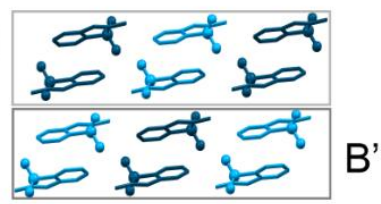

Figure S8. Crystal packing comparison for predictions showing the common similarity B, 2-Me04, 2-Me09. The packing instances are represented along two perpendicular directions, the molecules are colour coded according to the different orientation. Relative shifts of adjacent 2-D similarities are indicated by red arrows. These identify hypothetical phase transitions between the structures compared. 


\section{S4.3. Similarity C}

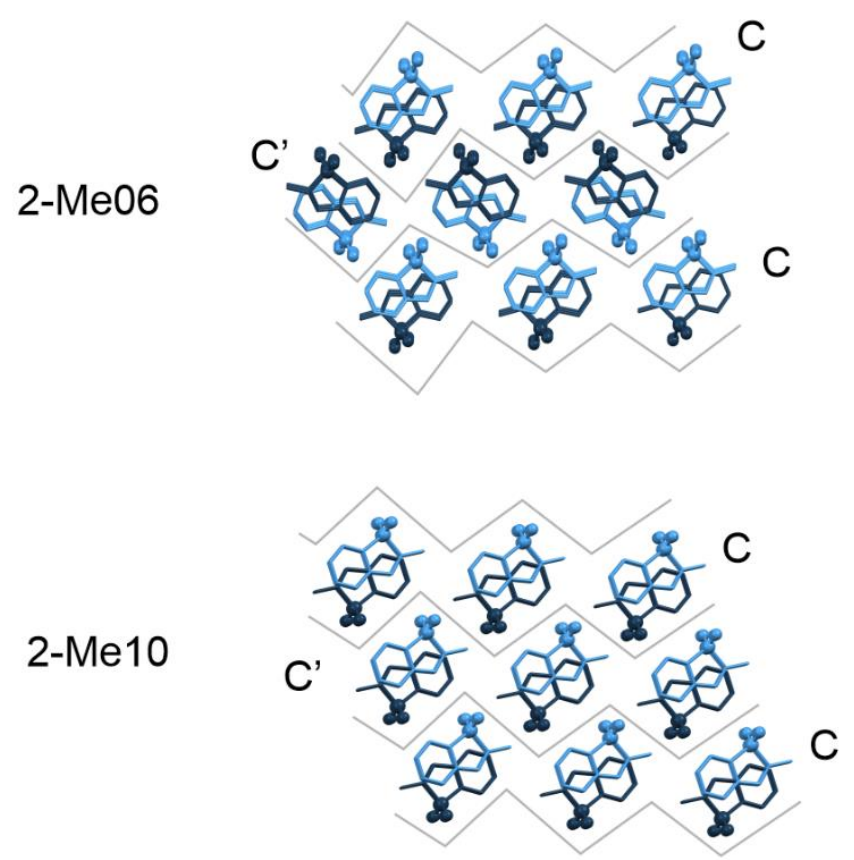

Figure S9 Crystal packing comparison for predictions showing the common similarity C, 2-Me06, 2-Me10. The molecules are colour coded according to the different orientation.

\section{S4.4. Similarity F}
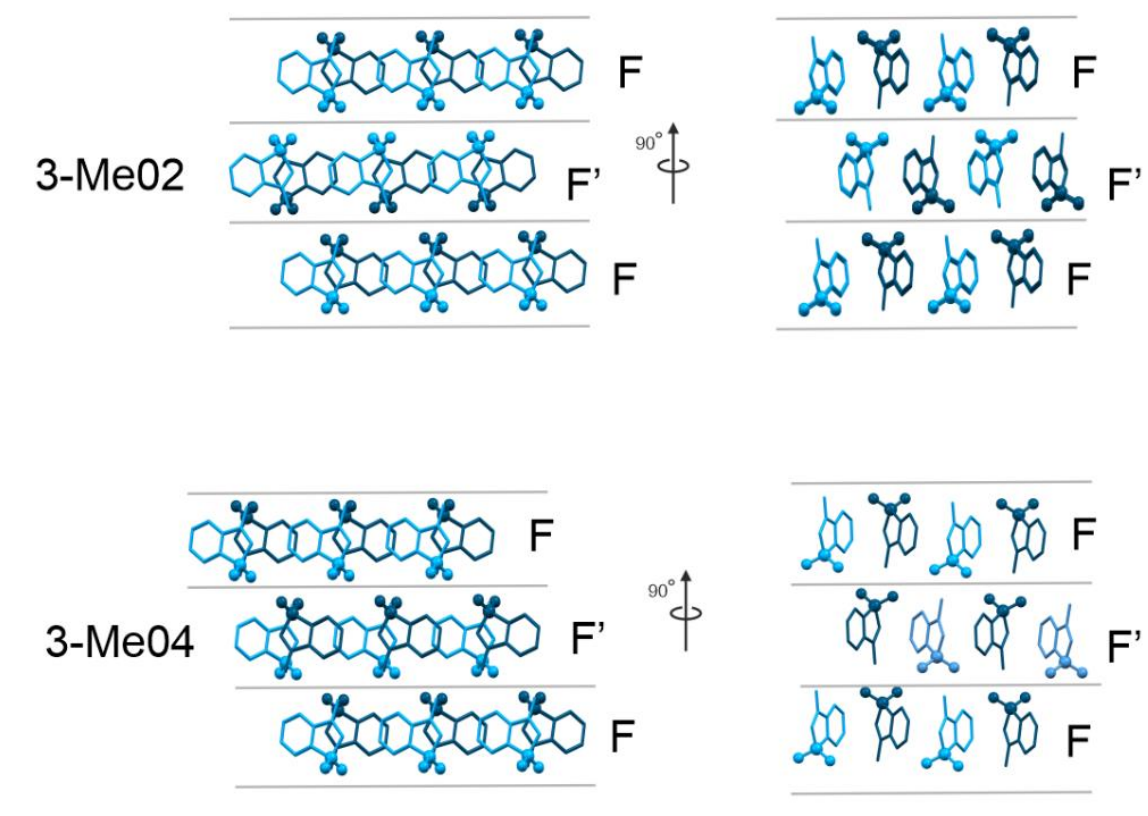

Figure S10.Crystal packing comparison for predictions showing the common similarity F, 3-Me02, 3-Me04. The molecules are colour coded according to the different orientation. 


\section{S4.5. Similarity I}

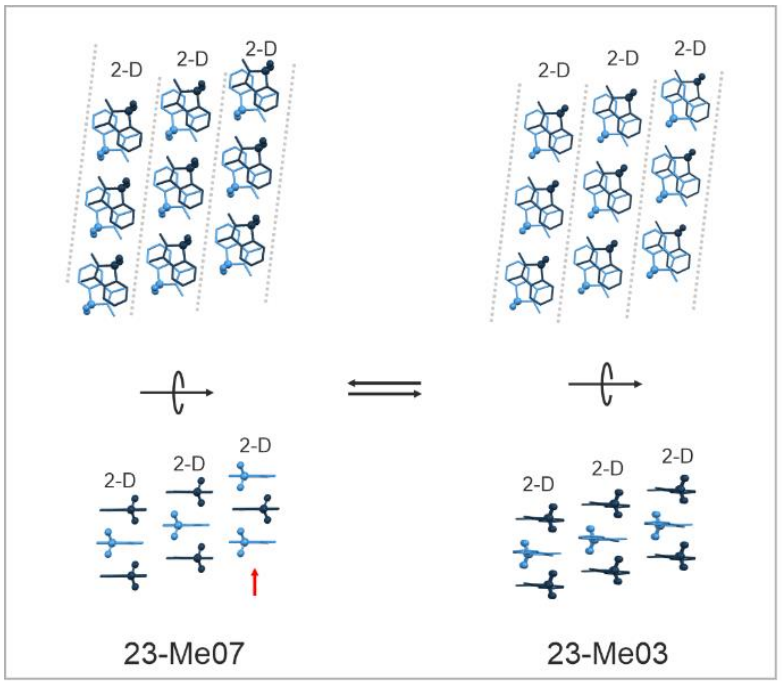

(a)

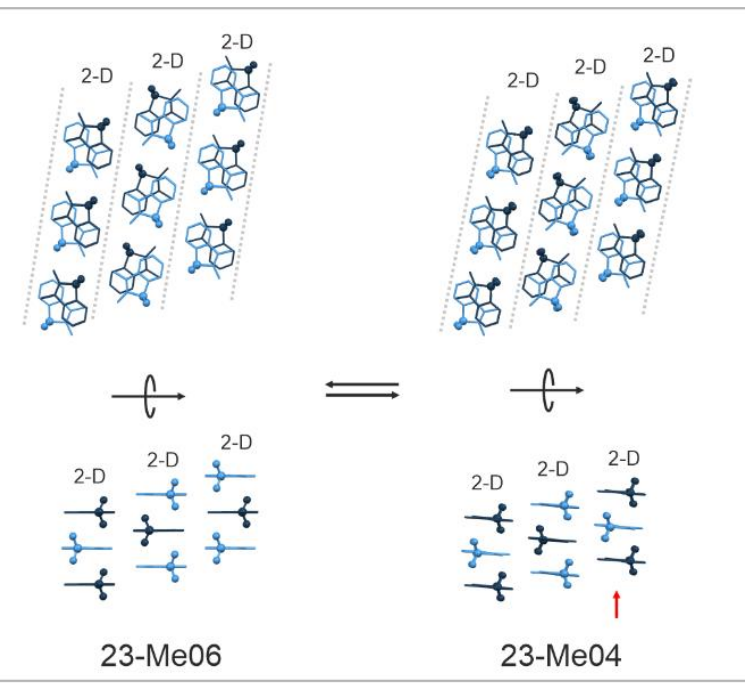

(b)

Figure S11. Crystal packing comparison for predictions showing the common similarity I. (a) 23-Me03, 23Me07; (b) 23-Me04, 23-Me06. The packing instances are represented along two perpendicular directions, the molecules are colour coded according to the different orientation. Different instances of the similarity I are indicated by grey dotted lines. Relative shifts of adjacent 2-D similarities are indicated by red arrows. These identify hypothetical phase transitions between the structures compared.

\section{References}

1. Kearney, P. C.; Mizoue, L. S.; Kumpf, R. A.; Forman, J. E.; McCurdy, A.; Dougherty, D. A. J. Am. Chem. Soc. 1993, 115, 9907-9919.

2. Geneste, P.; Grimaud, J.; Olive, J. L.; Ung, S. N., Bull. Soc. Chim. Fr. 1977, $271-275$.

3. Geneste, P.; Olive, J. L.; Ung, S. N.; Elamoudielfaghi, M.; Easton, J. W.; Bierbeck, H.; Saunders, J. K. J. Org. Chem. 1979, 44, 2887-2892.

4. Chabert, J. F. D.; Joucla, L.; David, E.; Lemaire, M. Tetrahedron 2004, 60, 3221-3230.

5. El Yahyaoui, A.; Felix, G.; Heynderickx, A.; Moustrou, C.; Samat, A. Tetrahedron 2007, 63, 94829487.

6. $\quad$ Buchwald, S. L.; Qun, F. J. Org. Chem. 1989, 54, 2793-2797.

7. El Amoudi El Faghi, M. S.; Geneste, P.; Olivé, J. L.; Rambaud, J.; Declercq, J.-P. Comparison of XRay Structure of 2- and 3-Methylbenzo[b]Thiophene 1,1-Dioxides. Acta Crystallogr. Sect. C 1988, 44 (3), $498-$ 500. https://doi.org/10.1107/S0108270187010680. 